\title{
Lymph node staging in early-stage ovarian cancer
}

Citation for published version (APA):

Kleppe, M. (2016). Lymph node staging in early-stage ovarian cancer. [Doctoral Thesis, Maastricht University]. GVO drukkers \& vormgevers B.V. https://doi.org/10.26481/dis.20161215mk

Document status and date:

Published: 01/01/2016

DOI:

10.26481/dis.20161215mk

Document Version:

Publisher's PDF, also known as Version of record

\section{Please check the document version of this publication:}

- A submitted manuscript is the version of the article upon submission and before peer-review. There can be important differences between the submitted version and the official published version of record.

People interested in the research are advised to contact the author for the final version of the publication, or visit the DOI to the publisher's website.

- The final author version and the galley proof are versions of the publication after peer review.

- The final published version features the final layout of the paper including the volume, issue and page numbers.

Link to publication

\footnotetext{
General rights rights.

- You may freely distribute the URL identifying the publication in the public portal. please follow below link for the End User Agreement:

www.umlib.nl/taverne-license

Take down policy

If you believe that this document breaches copyright please contact us at:

repository@maastrichtuniversity.nl

providing details and we will investigate your claim.
}

Copyright and moral rights for the publications made accessible in the public portal are retained by the authors and/or other copyright owners and it is a condition of accessing publications that users recognise and abide by the legal requirements associated with these

- Users may download and print one copy of any publication from the public portal for the purpose of private study or research.

- You may not further distribute the material or use it for any profit-making activity or commercial gain

If the publication is distributed under the terms of Article $25 \mathrm{fa}$ of the Dutch Copyright Act, indicated by the "Taverne" license above, 


\section{Lymph node staging in early-stage ovarian cancer}

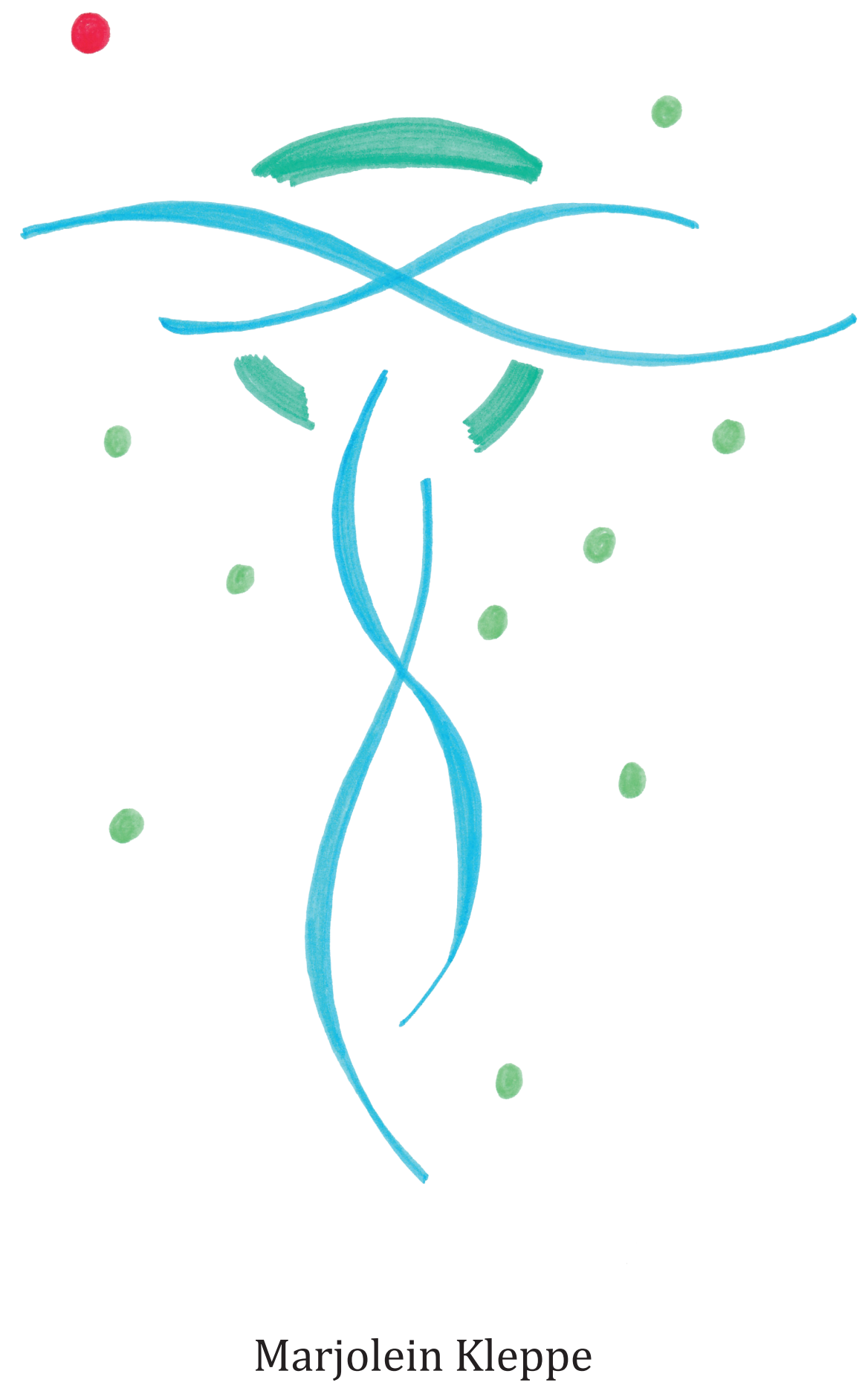



Lymph node staging in early-stage ovarian cancer

Marjolein Kleppe

(C) Marjolein Kleppe

ISBN: 978-94-6332-097-9

Lay-out: GVO drukkers

Printed by: GVO drukkers

Cover: Sabine Boertjens 



\title{
Lymph node staging in early-stage ovarian cancer
}

\author{
PROEFSCHRIFT
}

ter verkrijging van de graad van doctor aan de Universiteit Maastricht, op gezag van de Rector Magnificus, Prof. dr. Rianne M. Letschert volgens het besluit van het College van Decanen, in het openbaar te verdedigen op donderdag 15 december 2016 om 10.00 uur

door

\section{Marjolein Kleppe}




\section{Promotor}

Prof. dr. R.F.P.M. Kruitwagen

\section{Copromotoren}

Dr. T. van Gorp

Dr. B.F.M. Slangen

\section{Beoordelingscommissie}

Prof. Dr. G.L. Beets (voorzitter)

Antoni van Leeuwenhoek, Nederlands Kanker Instituut

Prof. Dr. F.M. Mottaghy

Prof. Dr. M.J.E. Mourits

Universitair Medisch Centrum Groningen

Prof. Dr. V.C.G. Tjan- Heijnen

Prof. Dr. J.B.M.Z. Trimbos

Leids Universitair Medisch Centrum 


\section{Contents}

Page

$\begin{array}{lll}\text { Chapter } 1 \text { Introduction } & 9\end{array}$

Chapter 2 Lymph node metastasis in stage I and II ovarian cancer: 21 a review.

Kleppe M, Wang T, Van Gorp T, Slangen BFM, Kruse AJ, Kruitwagen RFPM.

Gynecol Oncol 2011;123:610-4.

Chapter 3 Lymph-node metastasis in stage I and II sex cord stromal and malignant germ cell tumours of the ovary: a systematic review.

Kleppe M, Amkreutz LC, Van Gorp T, Slangen BFM, Kruse AJ, Kruitwagen RFPM.

Gynecol Oncol 2014;133:124-7.

Chapter 4 Sentinel node in ovarian cancer: study protocol for a phase

1 study.

Kleppe M, Van Gorp T, Slangen BFM, Kruse AJ, Brans B, Pooters INA, Van de Vijver KK, Kruitwagen RFPM.

Trials 201315;14:47.

Chapter 5 The detection of sentinel nodes in ovarian cancer: a feasibility 67 study.

Kleppe M, Brans B, Van Gorp T, Slangen BFM, Kruse AJ, Pooters INA, Lotz MG, Van de Vijver KK, Kruitwagen RFPM. J Nucl Med 2014;55:1799-804.

Chapter 6 Understanding lymphatic drainage pathways of the ovaries to 89 predict sites for sentinel nodes in ovarian cancer.

Kleppe M, Kraima AC, Kruitwagen RFPM, Van Gorp T, Smit NN, van Munsteren JC, DeRuiter MC. Int J Gynecol Cancer 2015;25:1405-14.

Chapter 7 The impact of lymph node dissection and adjuvant chemotherapy on survival: a nationwide cohort study of patients with clinical early-stage ovarian cancer. Kleppe $M$, van $\operatorname{der} A a M$, Van Gorp T, Slangen BFM, Kruitwagen RFPM. Eur J Cancer 2016;66:83-90.

Chapter 8 Valorisation addendem 
Chapter 10 Summary/ Nederlandse samenvatting

Chapter 11 List of co-authors

List of publications

Curriculum Vitae

Dankwoord 



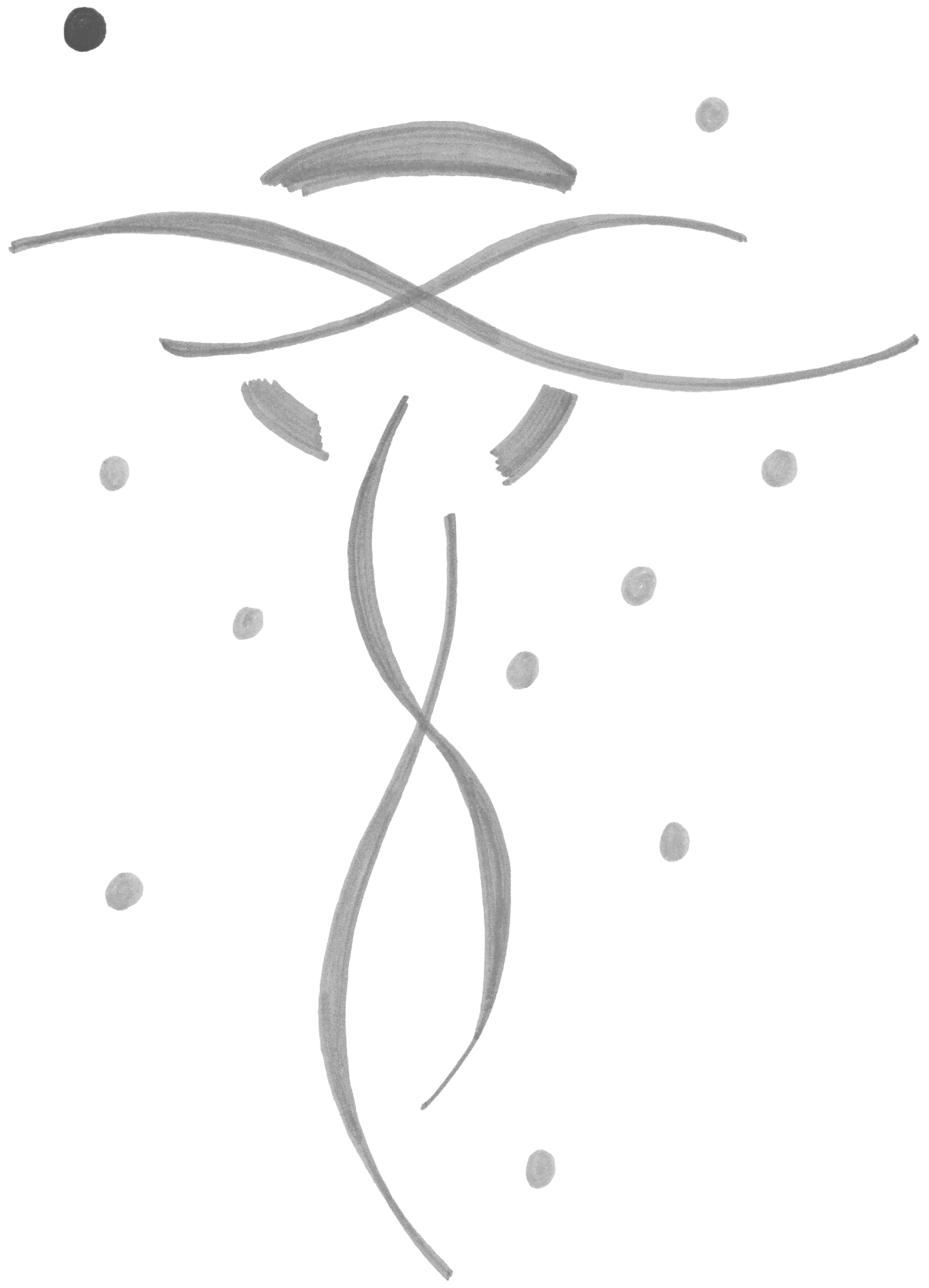




\section{Chapter 1}

\section{Introduction}




\section{Introduction}

Ovarian cancer is not the most common type of the female reproductive cancers, but it is the gynaecologic malignancy with the highest case-to-fatality ratio. This is related to the fact that ovarian cancer is hard to detect early. Women with ovarian cancer may have no symptoms or just mild symptoms until the disease is in an advanced stage.

\section{Histological classification of malignant ovarian tumours.}

Ovarian malignancies exhibit a wide variety of histological features. The histological classification by the World Health Organization (WHO) is based on histogenetic principles, and this classification categorizes ovarian tumours with regard to their derivation from epithelial cells, germ cells, and mesenchyme (the stroma and the sex cord).

\begin{tabular}{ll}
\hline Epithelial tumours & Non-epithelial tumours \\
\hline - serous & Germ cell tumours \\
- mucinous & - dysgerminoma \\
- endometrioid & - yolk sac \\
- clear cell & - choriocarcinoma \\
- transitional (including Brenner) & - embryonal carcinoma \\
- squamous cell & - immature teratoma \\
- undifferentiated & \\
& \\
\hline & Sex cord stromal tumours \\
& - granulosacell \\
& - Sertoli-Leydigcell \\
\end{tabular}




\section{Epithelial ovarian cancer}

Epithelial ovarian cancer can be divided in invasive epithelial ovarian cancer and so called borderline tumours (also known as atypically proliferating or low malignant potential). The theory on the origin of epithelial ovarian cancer has changed in recent years. Traditionally, epithelial ovarian cancer was thought to arise from damaged ovarian epithelium. However, recently, studies suggest the fallopian tube as the source of epithelial ovarian cancer, since precursors of highgrade carcinoma can be found in the uterine tube (serous tubal intra-epithelial carcinoma). ${ }^{1}$ The fimbriae, which are in close contact with the ovaries, are the preferred site of serous tubal intra-epithelial carcinoma.

Invasive epithelial ovarian cancer is the tumour with the most unfavourable prognosis within the field of the gynaecological oncology. In 2014 in the Netherlands 1272 women were diagnosed with invasive epithelial ovarian cancer. In the period of 2008 till 2012 the five-year survival was 38\%.2 Epithelial ovarian cancer is mostly found in patients of 65-69 years, and 25\% are 70 years or older. ${ }^{2}$ The high mortality rate is mainly due to the fact that approximately $75 \%$ of the patients are diagnosed with advanced stage epithelial ovarian cancer.

\section{Non-epithelial ovarian cancer}

Non-epithelial ovarian cancer accounts for approximately $10 \%$ of all malignant ovarian tumours and is classified into the ovarian sex cord stromal tumours, ovarian germ cell tumours and mesenchymal tumours. ${ }^{3,4}$

Sex cord stromal tumours arise from the dividing cells that would typically give rise to specialized gonadal stroma surrounding the oocytes, including the granulosacell, theca cells and Sertoli-Leydig cells. ${ }^{5}$

Sex cord stromal tumours have a good long-term prognosis, because most tumours are diagnosed in an early-stage. The average age at diagnosis of adult granulosa cell tumours is 50 years. Juvenile granulosa cell tumours can present at a younger age but account for only $5 \%$ of cases. ${ }^{5}$ Sertoli-Leydig cell tumours usually occur under the age of 40.6

Germ cell tumours are rapidly growing, predominantly unilateral tumours. They 
are mostly found in girls and young women and cure rates are high, also because of their high sensitivity for chemotherapy. The current platinum-based chemotherapy regimes used in children are associated with excellent survival rates, even in advanced stage disease. Therefore, a fertility-sparing approach is often considered. $^{7}$

\section{Surgical staging in ovarian cancer}

Ovarian cancer can metastasize through three different ways: intraperitoneal (in the abdominal cavity), lymphogenous and, less frequently, haematogenous. ${ }^{8-9}$ Concerning the lymphogenous spread, it is clear that lymphatic metastases of ovarian cancer mainly occur in the para-aortic lymph nodes. ${ }^{6}$ It is believed that the tumour cells follow the lymph vessels that accompany the ovarian artery and vein in the infundibulopelvic ligament up to the high para-aortic region and renal vein. Nevertheless, pelvic lymph node metastases are also frequently found. ${ }^{10}$ These tumour cells probably follow a different route, following the para-uterine vessels in the broad ligament towards the uterine artery and vein and further on to the iliac vessels. Isolated inguinal node metastases are also described.11 The exact mechanism of this route of metastasis is still unclear, but the metastatic cells might follow the course of the round ligament towards the inguinal lymph nodes, or might follow the iliac vessels towards the femoral vessels. Accurate knowledge of the lymphatic drainage pathways of the ovaries is essential to understand possible routes of lymphatic metastasis in ovarian cancer.

According to the International Federation of Gynaecology and Obstetrics (FIGO), ovarian cancer with lymph node metastases is classified as FIGO stage IIIA disease for which adjuvant chemotherapy is indicated. ${ }^{12}$

\section{Surgical staging in clinical early-stage epithelial ovarian cancer}

The diagnosis early-stage invasive epithelial ovarian cancer is based on the pathological results of a surgical staging procedure which consist of peritoneal washings for cytology, bilateral salgino-oophorectomy, hysterectomy, infracolic omentectomy, biopsy of the diaphragmatic peritoneum, paracolic gutters, pelvic peritoneum, and a systematic pelvic and para-aortic lymphadenectomy. 
Because lymph node metastases can be missed during lymph node sampling, systematic lymphadenectomy is considered the gold standard. FIGO recommends a complete para-aortic and pelvic lymphadenectomy in epithelial ovarian cancer. ${ }^{12} \mathrm{~A}$ lymphadenectomy is a radical procedure that is associated with morbidity, including nerve and vessel injury, increased blood loss, increased operating time, and the formation of lymphocysts and lymphedema. ${ }^{13-15}$ This probably is one of the reasons that surgical staging of ovarian cancer and the extent of lymph node dissection differs greatly from centre to centre and from country to country. ${ }^{16}$

The Dutch guideline recommends an adequate lymph node sampling, with an absolute minimum of ten lymph nodes removed. ${ }^{17}$ These lymph nodes also need to be sampled from different anatomical regions, of which the most important are the para-aortic and paracaval region between the renal vein and inferior mesenteric artery, the common, internal and external iliac vessels and the obturator fossa. In the same guideline, a footnote is made stating that a larger number of removed lymph nodes will increase the chance of finding metastases. ${ }^{17}$

The role of adjuvant chemotherapy in adequately staged early-stage invasive epithelial ovarian cancer is still controversial. ${ }^{18,19}$ Some studies have indicated that, irrespective of the extensiveness of a staging procedure, adjuvant chemotherapy improves survival. ${ }^{20-22}$

\section{Surgical staging in clinical early-stage non-epithelial ovarian cancer}

In clinical early-stage non-epithelial ovarian cancer, the recommended surgical treatment is similar to the surgical staging procedure in epithelial ovarian cancer. ${ }^{12}$ Despite this FIGO recommendation, many patients with non-epithelial ovarian cancer are not completely staged.

Lymphadenectomy or lymph-node sampling may not be performed for the same reasons as previously mentioned for invasive epithelial ovarian cancer. Moreover, since non-epithelial ovarian cancer is common in premenopausal patients, the diagnosis is often made after an operation for a presumed benign ovarian mass. Re-operating the patient for staging purposes is often not performed because, in case of a granulosacell tumour, the incidence of 
lymph node metastases is believed to be very low or, in case of a germ cell tumour, an extensive staging procedures is preferably avoided because of future fertilitywish and a high sensitivity of the tumour for chemotherapy. ${ }^{23}$

\section{Sentinel node technique in ovarian cancer}

A sentinel node procedure enables the identification of the first lymph node receiving primary lymphatic flow (the so-called sentinel node). In theory, the sentinel node technique could result in a high sensitivity with respect to the identification of lymph node metastases without the morbidity related to a complete lymphadenectomy. The sentinel node technique has been proven effective in vulvar and breast cancer, and sentinel studies in cervical and uterine cancers are currently under way. ${ }^{24-26}$ For the ovary, sentinel node studies are scarce, partly because of the risk of tumour dissemination associated with the injection of tracers into the ovarian cortex. ${ }^{27,28}$ Two studies, in which tracers were injected in the ovarian cortex, were performed in patients with endometrial cancer en macroscopically normal ovaries. ${ }^{27,}{ }^{28}$ In nearly all patients (11 out of 11 , and 15 out of 16) one or more sentinel nodes were identified. To prevent the risk of tumour spillage by injecting tracers into the ovarian cortex in case of an ovarian malignancy, the ovarian ligaments could be a good alternative site for injection of the tracers. However, few studies have investigated the lymphatic drainage pathways of the ovaries. $^{29,30}$

\section{Aims and outline}

This thesis focuses on (clinical) early-stage ovarian cancer. As with all cancers, early treatment of ovarian cancer is important. In case of clinical early-stage ovarian cancer surgery plays an important role with respect to the final diagnosis, staging and treatment. Staging is important because ovarian cancer has different prognosis at different stages and are treated differently. Inadequate staging can cause under diagnosis of advanced stage ovarian cancer and therefor under treatment. However, the necessity and extensiveness of a surgical staging procedure, especially with respect to the lymph nodes, is subject of debate in literature. 
We wanted to answer the following questions:

- What is the incidence of lymph node metastases in clinical early-stage ovarian cancer?

In chapter two a review is presented on the incidence of lymph node metastases in clinical stages I and II invasive epithelial ovarian cancer. The influence of different histological subtypes and differentiation grade is examined. Furthermore, we analyse the incidence of both ipsilateral and contralateral pelvic nodes metastases in a macroscopically unilateral tumour.

In chapter three a review is presented on the incidence of lymph-node metastasis in clinical stage I and II non-epithelial ovarian cancer.

- Is the sentinel node technique feasible in ovarian cancer?

Chapter four describes a study protocol for a feasibility study focussing on sentinel node detection in ovarian cancer. The aim of the study is to determine the feasibility of the sentinel node procedure performed by the injection of tracers into the ovarian ligaments and to establish whether the procedure is safe for the healthcare workers. The results of this study are discussed in chapter five.

- What is the anatomical course of the lymph drainage of the ovaries?

In chapter six the lymphatic drainage pathways of the ovaries in three human foetal pelves is studied. Serial transverse sections were produced and stained from which three-dimensional figures were constructed. Implications of these findings for the surgical management of epithelial ovarian cancer are also discussed.

- What is the impact of lymph node dissection and adjuvant chemotherapy on the survival in early-stage invasive epithelial ovarian cancer?

Chapter seven is a nationwide cohort study that describes the impact of lymph node dissection and adjuvant chemotherapy on the survival of patients with earlystage invasive epithelial ovarian cancer. All patients with a stage I-IIA and IIIA1 epithelial ovarian cancer in the Netherlands in the period from 2000 up to 2012 were included, in total 3658 patients. 


\section{References}

1. Kurman RJ, Shih IM. Mocular pathogenesis and extraovarian origin of epithelial ovarian cancer- Shifting the paradigm. Human pathology 2011;42:918-931.

2. www.cijfersoverkanker.nl

3. Prat J, Mutch DG. 2014 FIGO staging for ovarian, fallopian tube and peritoneal cancer. Gynecol Oncol 2014;133:401-404.

4. Gershenson DM. Current advances in the management of malignant germ cell and sex cord-stromal tumours of the ovary. Gynecol Oncol 2012;125:515-517.

5. Kurman, R.J., Carcangiu, M.L., Herrington, C.S., Young, R.H. World Health Organization Classification of tumours of female reproductive organs. Kurman R.J. $4^{\text {th }}$ revised edition. IARC Press: Lyon 2014.

6. Colombo N, Peiretti M, Garbi A, Carinelli S, Marini C, Sessa C. Non-epithelial ovarian cancer: ESMO clinical practice guidelines for diagnosis, treatment and follow-up. Ann Oncol 2012;23:20-26.

7. Madhi H, Swensen RE, Hanna E, Kumar S, Ali-Fehmi R, Semaan A, Tamimi H, Morris RT, Munkarah AR. Prognostic impact of lymphadenectomy in clinical early stage malignant germ cell tumour of the ovary. Br J Cancer 2011;105:493-497.

8. Panici PB, Angioli R. Role of lymphadenectomy in ovarian cancer. Best Pract Res Clin Obstet Gynaecol 2002;16:529-551.

9. Tan DS, Agarwal R, Kaye SB. Mechanisms of transcoelomic metastasis in ovarian cancer. Lancet Oncol 2006;7:925-934.

10. Burghardt E, Girardi F, Lahousen M, Tamussino K, Stettner H. Patterns of pelvic and paraaortic lymph node involvement in ovarian cancer. Gynecol Oncol 1991;40:103-106.

11. Ang D, Ng KY, Tan HK, Chung AY, Yew BS, Lee VK. Ovarian carcinoma presenting with isolated contralateral inguinal lymph node metastasis: a case report. Ann Acad Med Singapore 2007;36:427-30.

12. Prat J, Mutch DG. 2014 FIGO staging for ovarian, fallopian tube and peritoneal cancer. Gynecol Oncol 2014;133:401-404. 
13. Ditto A, Martinelli F, Reato C, Kusamura S, Solima E, Fontanelli R, Haeusler E, Raspagliesi F. Systematic para-aortic and pelvic lymphadenectomy in early stage epithelial ovarian cancer: a prospective study. Ann Surg Oncol 2012;19:3849-3855.

14. Maggioni A, Benedetti Panici P, Dell'Anna T, Landoni F, Lissoni A, Pellegrino A, Rossi RS, Chiari S, Campagnutta E, Greggi S, Angiolo R, Manci N, Calcagno M, Scambia G, Fossati R, Floriani I, Torri, Grassi R, Mangioni C. Randomised study of systematic lymphadenectomy in patients with epithelial ovarian cancer macroscopically confined to the pelvis. British J of Cancer 2006;95:699-704.

15. Van de Poll-Franse L, Pijnenborg JMA, Boll D, Vos MC, van den Berg H, Lybeert ML, de Winter K, Kruitwagen RF. Health related quality of life and symptoms after pelvic lymphadectomy or radiotherapy vs. no adjuvant regional treatment in early-stage endometrial carcinoma: a large population-based study. Gynecol Oncol 2012;127:153-160.

16. Carnino F, Fuda G, Ciccone G, Iskra L, Guercio E, Dadone D, Conte PF. Significance of lymph node sampling inepithelial carcinoma of the ovary. Gynecol Oncol 1997;65:467-472.

17. http://www.oncoline.nl/ovariumcarcinoom

18. Tropé C, Kaern J. Adjuvant chemotherapy for early-stage ovarian cancer: review of the literature. J Clin Oncol 2007;25:2909-2920.

19. Cress RD, Bauer K, O’Malley CD Kahn AR, Schymura MJ, Stewart SL, Leiserowitz GS. Surgical staging of early stage epithelial ovarian cancer: results from the CDC-NPCR ovarian patterns of care study. Gynecol Oncol 2011;121:94-99.

20. Trimbos JB, Parmar M, Vergote I, Guthrie D, Bolis G, Colombo N, Vermorken JB, Torri V, Mangioni C, Pecorelli S, Lissoni A, Swart AM. International collaborative ovarian neoplasm trial 1 and adjuvant chemotherapy in ovarian neoplasm trial: two parallel randomized phase III trials of adjuvant chemotherapy in patients with early-stage ovarian carcinoma. J Natl Cancer Inst 2003;95:105-112.

21. Colombo N, Guthrie D, Chiari S Parmar M, Qian W, Swart AM, Torri V, Williams C, Lissoni A, Bonazzi C; International Collaborative Ovarian 
Neoplasm (ICON) collaborators. International collaborative ovarian neoplasm trial 1: a randomised trial of adjuvant chemotherapy in women with early-stage ovarian cancer. J Natl Cancer Inst 2003;95:125-132.

22. Trimbos JB, Vergote I, Bolis G, Vermorken JB, Mangioni C, Madronal C, Franchi M, Tateo S, Zanetta G, Scarfone G, Giurgea L, Timmers P, Coens C, Pecorelli S; EORTC-ACTION collaborators. Impact of adjuvant chemotherapy and surgical staging in early-stage ovarian carcinoma: European Organisation for Research and Treatment of Cancer- Adjuvant chemotherapy in ovarian neoplasm trial. J Natl Cancer Inst 2003;95:113124.

23. Brown J, Sood AK, Deavers MT, Milojevic L, Gershenson DM. Patterns of metastasis in sex cord-stromal tumours of the ovary: Can routine staging lymphadenectomy be omitted? Gynecol Oncol 2009;113:86-90.

24. de Hullu JA, Hollema H, Piers DA, Verheijen RH, van Diest PJ, Mourits MJ, Aalders JG, van Der Zee AG. Sentinel lymph node procedure is highly accurate in squamous cell carcinoma of the vulva. J Clin Oncol 2000;18:2811-2816.

25. Mansel RE, Fallowfield L, Kissin M, Goyal A, Newcombe RG, Dixon JM, Yiangou C, Horgan K, Bundred N, Monypenny I, England D, Sibbering M, Abdullah TI, Barr L, Chetty U, Sinnett DH, Fleissig A, Clarke D, Ell PJ. Randomized multicenter trial of sentinel node biopsy versus standard axillary treatment in operable breast cancer: the ALMANAC Trial. J Natl Cancer Inst 2006;98:599-609.

26. El-Ghobashy AE, Saidi SA. Sentinel node sampling in gynaecological cancers: techniques and clinical applications. Eur J Surg Onc 2009;35:675-685.

27. Negishi H, Takeda M, Fujimoto T, Todo Y, Ebina Y, Watari H, Yamamoto R, Minakami H, Sakuragi N. Lymphatic mapping and sentinel node identification as related to the primary sites of lymph node metastasis in early stage ovarian cancer. Gynecol Oncol 2004;94:161-166.

28. Nyberg RH, Korkola P, Tech L, Mäenpää J. Ovarian sentinel node, is it feasible? Int J Gynecol Cancer 2011;21:567-572.

29. Eicher E, Bove ER. In vivo studies on the lymphatic drainage of the human ovary. Obstet Gynecol 1954;3:287-297. 
30. Eicher E, Goldberg I, Bove ER. In vivo studies with direct sky blue of the lymphatic drainage of the internal genitals of women. Am J Obstet Gynecol. 1954;67:1277-1287. 


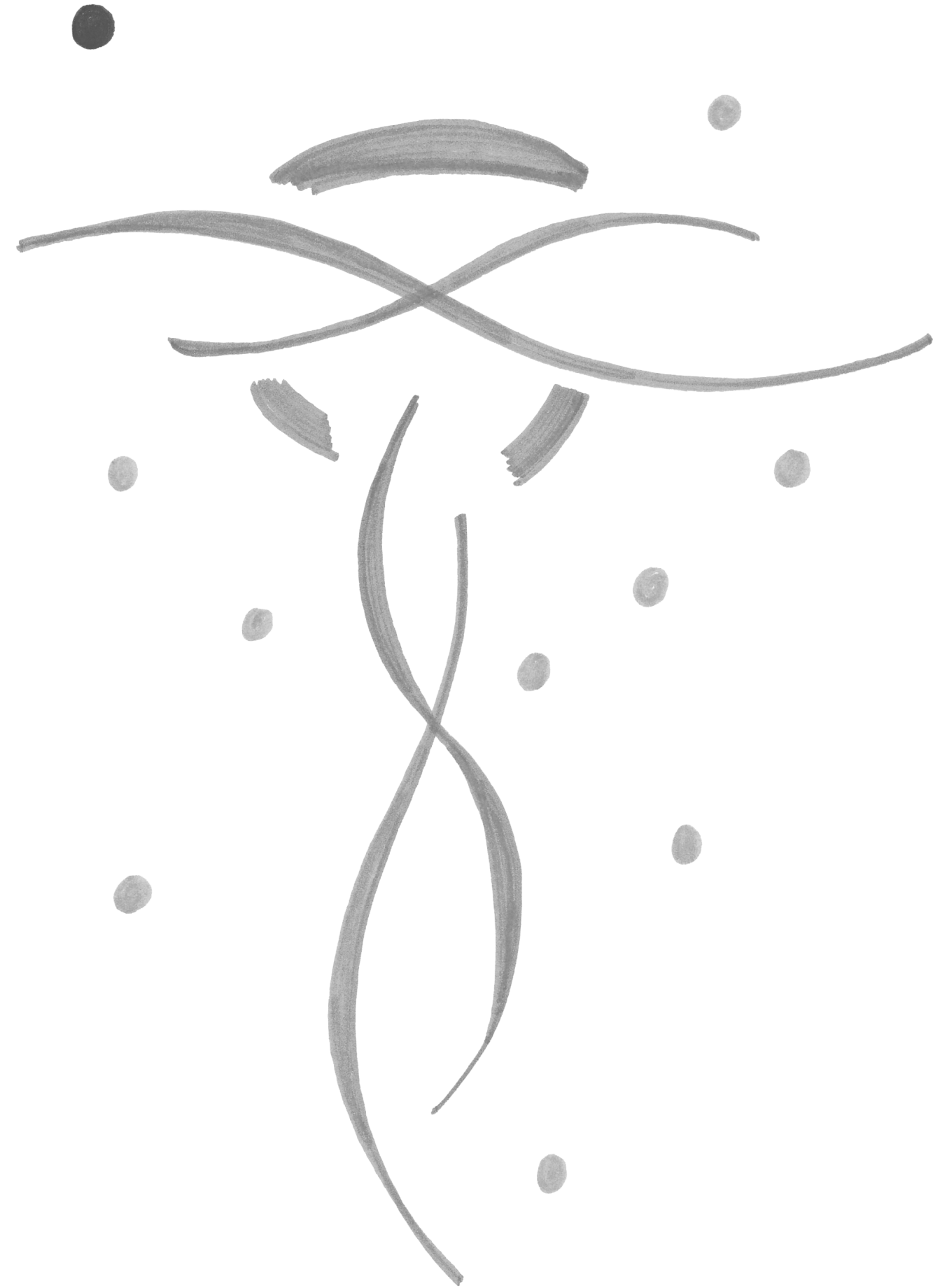




\section{Chapter 2}

\section{Lymph Node Metastasis}

in Stage I and II Ovarian

\section{Cancer: a Review}

Kleppe M, Wang T, Van Gorp T, Slangen BFM, Kruse AJ, Kruitwagen RFPM

Gynecologic Oncology 2011;123:610-614 


\begin{abstract}
Objectives:

The purpose of this review is to determine the incidence of lymph node metastases in clinical stage I and II ovarian cancer.
\end{abstract}

\title{
Methods:
}

Relevant articles were identified from MEDLINE and EMBASE, supplemented with citations from reference lists from the primary studies. Eligibility was evaluated by two authors. Included studies were prospective or retrospective cohort studies, which analyzed patients with clinical early-stage epithelial ovarian cancer (EOC) who underwent a complete pelvic and para-aortic lymphadenectomy as a part of a staging laparotomy.

\section{Results:}

Fourteen studies were included in the analysis. The mean incidence of lymph node metastases in clinical stage I-II EOC was 14.2\% (range 6.1-29.6\%), of which $6.7 \%$ only in the para-aortic region, $3.3 \%$ only in the pelvic region, and $4.3 \%$ both in the para-aortic and pelvic region. Grade 1 tumours had a mean incidence of lymph node metastases of $4.0 \%$, grade 2 tumours $16.5 \%$ and grade 3 tumours 20.0\%. According to histological subtype, the highest incidence of lymph node metastases was found in the serous subtype (23.3\%), the lowest in the mucinous subtype (2.6\%). In unilateral tumours, pelvic lymph node metastases were found in $9.7 \%$ on both sides, $8.3 \%$ only at the ipsilateral side, and in $3.5 \%$ only at the contralateral side.

\section{Conclusions:}

The incidence of lymph node metastases in clinical early-stage EOC is considerable. Based on the scarce literature data, omitting a systematic lymphadenectomy can only be considered in grade I mucinous tumours. 


\section{Introduction}

Epithelial ovarian cancer (EOC) remains the tumour with the most unfavorable prognosis within the field of the gynaecological oncology. The incidence of ovarian cancer in the Netherlands in 2008 was 14.5 per 100.000 , with 12.3 deaths per $100.000 .{ }^{1}$ In the US in 2007 the incidence was 13.0 per 100.000 and there were 8.2 deaths per $100.000 .^{2}$ The high mortality rate is partially due to the fact that approximately $75 \%$ of patients is diagnosed with advanced stage EOC.

EOC can metastasize through three different ways: intraperitoneal (in the abdominal cavity), lymphogenous and haematogenous. ${ }^{3,4}$ Concerning the lymphogenous spread, it is clear that lymphatic metastases of EOC mainly occur in the para-aortic lymph nodes. ${ }^{5}$ It is believed that the tumour cells follow the lymph vessels that accompany the ovarian artery and vein in the infundibulopelvic ligament up to the high para-aortic region and renal vein. Nevertheless, pelvic lymph node metastases are also frequently found.6-16 These tumour cells probably follow a different route, following the para-uterine vessels in the broad ligament towards the uterine artery and vein and further on to the iliac vessels. In some case reports isolated inguinal node metastases are also described. ${ }^{17-20}$ The exact mechanism of this route of metastasis is still unclear, but the metastatic cells might follow the course of the round ligament towards the inguinal lymph nodes, or might follow the iliac vessels towards the femoral vessels.

According to the International Federation of Gynecology and Obstetrics (FIGO), EOC with lymph node metastases is classified as FIGO stage IIIC disease, even in the absence of peritoneal metastases. ${ }^{21}$ Surgical staging of EOC and the extent of lymph node dissection differs greatly from centre to centre. ${ }^{22}$

The Dutch guideline recommends an adequate lymph node sampling, with an absolute minimum of ten lymph nodes removed. In the same guideline, a footnote is made stating that a larger number of removed lymph nodes will increase the chance of finding metastases. ${ }^{1}$ These lymph nodes also need to be sampled from different anatomical regions, of which the most important are the 
paraaortic and paracaval region between the renal vein and inferior mesenteric artery, the common, internal and external iliac vessels and the obturator fossa.

In this review we aimed to identify the incidence of lymph node metastases in clinical stage I and II EOC and determine the influence of different histological subtypes and differentiation grade on the incidence of lymph nodes metastases. Furthermore, we analyzed the incidence of both ipsilateral and contralateral pelvic nodes metastases in a macroscopically unilateral tumour.

\section{Patients and methods}

A literature search was performed on MEDLINE and EMBASE. Specific searches were performed with the terms "ovarian neoplasm", "early-stage", "stage", "lymph nodes", "spread", "metastasis", "lymphadenectomy", "(para-) aortic", "pelvic", "dissection", "unilateral". Separate searches were performed with MeSH terms and clinical queries on MEDLINE to extract all relevant literature available. The searches were limited to "full text", "English" and "human". Additional literature was searched through cross-references of the retrieved articles.

The inclusion criteria (Figure 1):

1) Randomized controlled trials, and prospective and retrospective cohort studies.

2) Only studies that included patients with clinically FIGO stage I or II EOC or a subset of these specific groups were accepted.

3) A complete staging laparotomy including a systematic pelvic and paraaortic lymph node dissection (LND) had to be performed in all patients within the trial or cohort.

All abstracts of the studies obtained were evaluated by two authors (KM and WT) for eligibility. Full text was evaluated if the abstract did not provide enough information for the determination of eligibility. 
Figure 1. Literature search.
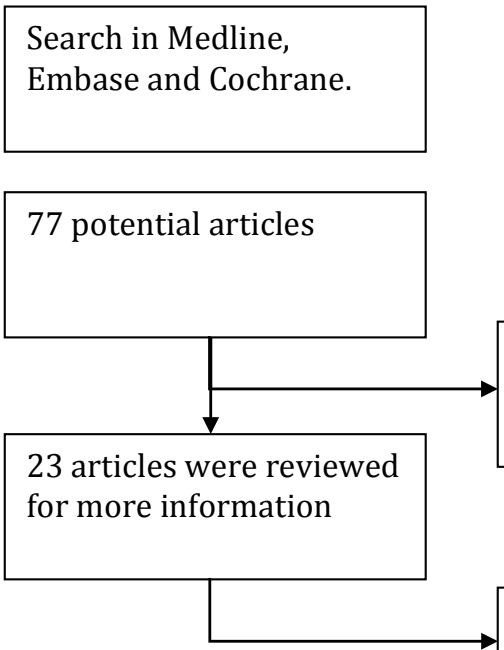

11 excluded:

53 articles excluded

based on title and abstract.

- 3 only included FIGO stage III - IV disease

- 2 only included random lymph node sampling

- 1 did not described the method of lymphadenectomy

- 3 reviews

- 2 did not perform a lymphadenectomy in all patients within the cohort

2 articles were added through cross reference search

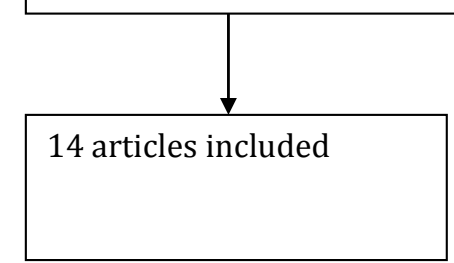




\section{Results}

Fourteen studies were included, of which two randomized controlled trials and 12 retrospective cohort studies. ${ }^{5-13,15,16,19,23,24}$ The mean number of pelvic and para-aortic lymph nodes removed ranged from 20 to 78. Nine studies did not describe the number of removed lymph nodes from the para-aortic and pelvic region separately. In the five studies that did describe the number of removed lymph nodes separately, the mean number of para-aortic and pelvic lymph nodes ranged from 15 to 46 and 14 to 74 , respectively (Table 1).

Table 1. Overall incidence of lymph node metastases in clinical FIGO stage I-II epithelial ovarian cancer and the anatomical distribution of positive lymph nodes.

\begin{tabular}{|c|c|c|c|c|c|c|c|c|c|c|c|c|}
\hline \multirow[t]{3}{*}{ Reference } & \multirow{3}{*}{$\begin{array}{c}\text { Clinical } \\
\text { FIGO } \\
\text { stage I-II } \\
\mathbf{n} \\
\end{array}$} & \multicolumn{11}{|c|}{ Positive pelvic and /or para-aortic lymph nodes } \\
\hline & & \multicolumn{2}{|c|}{ Overall } & \multicolumn{3}{|c|}{ Only para-aortic } & \multicolumn{3}{|c|}{ Only pelvic } & \multicolumn{3}{|c|}{$\begin{array}{l}\text { Both para-aortic } \\
\text { and pelvic }\end{array}$} \\
\hline & & $\mathbf{n}$ & \%* & $\mathbf{n}$ & $\% \S$ & \%* & $\mathbf{n}$ & $\% \S$ & \%* & n & $\% \S$ & $\%^{*}$ \\
\hline Ayhan et al. ${ }^{26}$ & 169 & 11 & 6.5 & 5 & 45.5 & 3.0 & 6 & 54.5 & 3.6 & 0 & 0.0 & 0.0 \\
\hline Benedetti et al. 6 & 37 & 5 & 13.5 & 2 & 40.0 & 5.4 & 3 & 60.0 & 8.1 & 0 & 0.0 & 0.0 \\
\hline Burgardt et al. 5 & 27 & 8 & 29.6 & 1 & 12.5 & 3.7 & 3 & 37.5 & 11.1 & 4 & 50.0 & 14.8 \\
\hline Desteli et al. ${ }^{24}$ & 33 & 2 & 6.1 & 1 & 50.0 & 3.0 & 1 & 50.0 & 3.0 & 0 & 0.0 & 0.0 \\
\hline Fournier et al. 19 & 108 & 19 & 17.6 & 10 & 52.6 & 9.3 & 5 & 26.3 & 4.6 & 4 & 21.1 & 3.7 \\
\hline Harter et al. 15 & 70 & 8 & 11.6 & 4 & 50.0 & 5,8 & 0 & 0.0 & 0.0 & 4 & 50.0 & 5.8 \\
\hline Morice et al. 11 & 100 & 23 & 23.0 & 13 & 56.5 & 13.0 & 3 & 13.0 & 3.0 & 7 & 30.4 & 7.0 \\
\hline Negishi et al. 12 & 150 & 19 & 12.7 & 14 & 73.7 & 9.3 & 2 & 10.5 & 1.3 & 3 & 15.8 & 2.0 \\
\hline Nomura et al. ${ }^{16}$ & 79 & 10 & 12.7 & 4 & 40.0 & 5.1 & 1 & 10.0 & 1.3 & 5 & 50.0 & 6.3 \\
\hline Onda et al. ${ }^{7}$ & 59 & 13 & 22.0 & 2 & 15.4 & 3.4 & 3 & 23.1 & 5.1 & 8 & 61.5 & 13.6 \\
\hline Saguraki et al. ${ }^{9}$ & 94 & 9 & 9.6 & 7 & 77.8 & 7.4 & 1 & 11.1 & 1.1 & 1 & 11.1 & 1.1 \\
\hline Suzuki et al. ${ }^{10}$ & 47 & 5 & 10.6 & 2 & 40.0 & 4.3 & 2 & 40.0 & 4.3 & 1 & 20.0 & 2.1 \\
\hline Takeshima et al. ${ }^{13}$ & 193 & 38 & 19.7 & 17 & 44.7 & 8.8 & 6 & 15.8 & 3.1 & 15 & 39.5 & 7.8 \\
\hline Tsumara et al. ${ }^{8}$ & 81 & 7 & 8.6 & 6 & 85.7 & 7.4 & 0 & 0.0 & 0.0 & 1 & 14.3 & 1.2 \\
\hline Total & 1247 & 177 & 14.2 & 88 & 49.7 & 7.1 & 36 & 20.3 & 2.9 & 53 & 29.9 & 4.3 \\
\hline
\end{tabular}

${ }^{*}$ The percentage indicates the number of patients with positive lymph nodes as a proportion of the total number of patients with clinical FIGO stage I-II tumours.

$\S$ The percentage indicates the number of patients with positive lymph nodes in that particular anatomical region as a proportion of the patients with positive lymph nodes.

FIGO: International Federation of Gynecology and Obstetrics

\section{Incidence of lymph node metastases in clinical FIGO stage I and II EOC}

The incidence of lymph node metastases in clinical FIGO stage I and II EOC is shown in Table 1. For the calculation of the overall incidence of lymph node metastases, 1246 patients from 14 different studies were included in the analysis. The mean incidence of lymph node metastases in clinical stage I-II EOC was $14.2 \%$ (range $6.1-29.6 \%$ ). The mean incidence of lymph node metastases 
was $6.7 \%$ (range $3.0-13.0 \%$ ) in the para-aortic region, 3.3\% (range $0.0-11.1 \%$ ) in the pelvic region and $4.3 \%$ (range $0.0-14.8 \%$ ) in both together.

Incidence of lymph node metastases according to differentiation grade and histological subtype of clinical FIGO stage I-II EOC.

For the calculation of lymph node metastases according to differentiation grade, 361 patients from 6 different studies were included (Table 2). The incidence of lymph node metastases in stages I-II is $4.0 \%$ in grade 1 tumours, $16.5 \%$ in grade 2 tumours and $20.0 \%$ in grade 3 tumours. For the calculation of lymph node metastases according to histological subtype, 574 patients from 8 different studies were included (Table 3). The incidence of metastatic lymph nodes was the highest in serous EOC (23.3\%), and the lowest in mucinous EOC (2.6\%). None of the studies that described lymph node metastases in mucinous tumours $(n=4)$, the differentiation grade was mentioned.7,8,12

When limiting the incidence according to grade and histological type to clinical FIGO stage I EOC, the incidence of lymph node metastases in grade 1, 2, and 3 tumours is respectively $2.9 \%, 13.8 \%$, and $20.0 \%(\mathrm{n}=188) .10,11,15,23$ According to histological subtype $(n=336)$, the incidence of metastatic lymph nodes again was the highest in serous EOC (19.3\%), and the lowest in mucinous EOC (1.9\%). ${ }^{10-}$ $12,15,23$

Table 2. Incidence of lymph node metastases in clinical stage I-II according to differentiation grade

\begin{tabular}{|c|c|c|c|c|c|c|c|c|c|c|c|}
\hline \multirow{3}{*}{ Reference } & \multirow{3}{*}{$\begin{array}{c}\begin{array}{c}\text { Total } \\
\text { population }\end{array} \\
\mathbf{n} \\
\end{array}$} & \multicolumn{3}{|c|}{ Grade 1} & \multicolumn{3}{|c|}{ Grade 2} & \multicolumn{3}{|c|}{ Grade 3} & \multirow{3}{*}{$\begin{array}{c}\begin{array}{c}\text { Missing } \\
\text { data }\end{array} \\
\mathbf{n} \\
\end{array}$} \\
\hline & & \multirow{2}{*}{$\frac{\text { Total }}{\mathbf{n}}$} & \multicolumn{2}{|c|}{$\mathrm{LN}+$} & \multirow{2}{*}{$\frac{\text { Total }}{\mathbf{n}}$} & \multicolumn{2}{|c|}{$\mathrm{LN}+$} & \multirow{2}{*}{$\frac{\text { Total }}{\mathbf{n}}$} & \multicolumn{2}{|c|}{$\mathrm{LN}+$} & \\
\hline & & & $\mathbf{n}$ & $\% *$ & & $\mathbf{n}$ & $\% *$ & & $\mathbf{n}$ & $\% *$ & \\
\hline Desteli et al. ${ }^{23}$ & 33 & 11 & 0 & 0.0 & 8 & 0 & 0.0 & 14 & 2 & 14.3 & \\
\hline Harter et al. 15 & 48 & 10 & 0 & 0.0 & 25 & 2 & 8.0 & 13 & 1 & 7.7 & \\
\hline Morice et al. ${ }^{11}$ & 60 & 15 & 0 & 0.0 & 21 & 4 & 19.0 & 4 & 4 & 100.0 & 20 \\
\hline Nomura et al. 16 & 79 & 21 & 1 & 4.8 & 13 & 3 & 23.1 & 6 & 2 & 33.3 & 39 \\
\hline Sakuragi et al. ${ }^{9}$ & 94 & 60 & 3 & 5.0 & 25 & 5 & 20.0 & 9 & 1 & 11.1 & \\
\hline Suzuki et al. ${ }^{10}$ & 47 & 32 & 2 & 6.3 & 11 & 3 & 27.3 & 4 & 0 & 0.0 & \\
\hline Total & 361 & 149 & 6 & 4.0 & 103 & 17 & 16.5 & 50 & 10 & 20.0 & 66 \\
\hline
\end{tabular}

LN+: lymph node metastases

* The percentage indicates the number of patients with positive lymph nodes in the mentioned grade as a proportion of the total number of patients of that grade. 
Table 3. Incidence of lymph node metastases according to histological subtype.

\begin{tabular}{|c|c|c|c|c|c|c|c|c|c|c|c|c|c|c|c|c|c|}
\hline \multirow{3}{*}{ Reference } & \multirow{3}{*}{$\begin{array}{c}\begin{array}{c}\text { Total } \\
\text { populatio } \\
\mathbf{n}\end{array} \\
\mathrm{n}\end{array}$} & \multirow{3}{*}{$\begin{array}{l}\text { FIGO } \\
\text { Stage }\end{array}$} & \multicolumn{3}{|c|}{ Serous } & \multicolumn{3}{|c|}{ Mucinous } & \multicolumn{3}{|c|}{ Endometriod } & \multicolumn{3}{|c|}{ Clear Cell } & \multicolumn{3}{|c|}{$\begin{array}{c}\text { Undifferentiated } \\
\text { Others }\end{array}$} \\
\hline & & & \multirow{2}{*}{$\begin{array}{c}\text { Total } \\
\mathrm{n}\end{array}$} & \multicolumn{2}{|c|}{ LN+ } & \multirow{2}{*}{$\begin{array}{c}\text { Total } \\
\mathrm{n}\end{array}$} & \multicolumn{2}{|c|}{ LN+ } & \multirow{2}{*}{$\frac{\text { Total }}{\mathrm{n}}$} & \multicolumn{2}{|c|}{ LN+ } & \multirow{2}{*}{$\frac{\text { Total }}{\mathrm{n}}$} & \multicolumn{2}{|c|}{ LN+ } & \multirow{2}{*}{$\begin{array}{c}\text { Total } \\
\mathrm{n}\end{array}$} & \multirow{2}{*}{\multicolumn{2}{|c|}{$\begin{array}{cc} & \text { LN+ } \\
\mathrm{n} & \% *\end{array}$}} \\
\hline & & & & $\mathrm{n}$ & $\% *$ & & $\mathrm{n}$ & $\% *$ & & $\mathrm{n}$ & $\% *$ & & $\mathrm{n}$ & $\% *$ & & & \\
\hline Desteli et al. ${ }^{23}$ & 33 & I & 7 & 1 & 14.2 & 8 & 0 & 0.0 & 5 & 0 & 0.0 & 4 & 0 & 0.0 & 9 & 1 & 11.1 \\
\hline Harter et al. ${ }^{15}$ & 48 & I & 13 & 2 & 15.4 & 8 & 0 & 0.0 & 14 & 1 & 7.1 & 7 & 0 & 0.0 & 6 & 0 & 0.0 \\
\hline Morice et al. 11 & 85 & I & 26 & 8 & 30.8 & 20 & 0 & 0.0 & 25 & 0 & 0.0 & - & - & - & 14 & 9 & 64.3 \\
\hline Negeishi et al. 12 & 150 & $\mathrm{I}-\mathrm{II}$ & 35 & 5 & 14.3 & 49 & 2 & 4.1 & 15 & 3 & 20.0 & 46 & 8 & 17.4 & 5 & 1 & 20.0 \\
\hline Nomura et al. 16 & 79 & I-II & 12 & 6 & 50.0 & 4 & 0 & 0.0 & 27 & 2 & 7.4 & 36 & 2 & 5.6 & - & - & - \\
\hline Onda et al. ${ }^{7}$ & 59 & I-II & 21 & 7 & 33.3 & 15 & 1 & 6.7 & 3 & 0 & 0.0 & 16 & 5 & 31.3 & 4 & 0 & 0.0 \\
\hline Suzuki et al. ${ }^{10}$ & 47 & I & 13 & 4 & 30.8 & 22 & 0 & 0.0 & 3 & 0 & 0.0 & 9 & 1 & 11.1 & 0 & 0 & 0.0 \\
\hline Tsumura et al. ${ }^{8}$ & 73 & I-II & 23 & 2 & 8.7 & 29 & 1 & 3.4 & - & - & - & 21 & 4 & 19.0 & - & - & - \\
\hline Total & 574 & & 150 & 35 & 23.3 & 155 & 4 & 2.6 & 92 & 6 & 6.5 & 139 & 20 & 14.4 & 38 & 11 & 28.9 \\
\hline
\end{tabular}

$\mathrm{LN}+$ : lymph node metastasis

* The percentage indicates the number of patients with positive lymph nodes in the mentioned histological type as a proportion of the total number of patients in that histological type.

FIGO: International Federation of Gynecology and Obstetrics

\section{Localization of lymph node metastases in the case of a unilateral tumour.}

Concerning unilateral clinical FIGO stage I-II EOC we were able to retrieve information on lymph node metastases from 7 different studies (Table 4). In case of lymph node metastases, they were localized only at the contralateral pelvic and para-aortic site in respectively $16.1 \%$ and $18.0 \%$.

Table 4. Incidence of pelvic and para-aortal lymph node metastases in patients with a macroscopic unilateral FIGO stage I-II tumour.

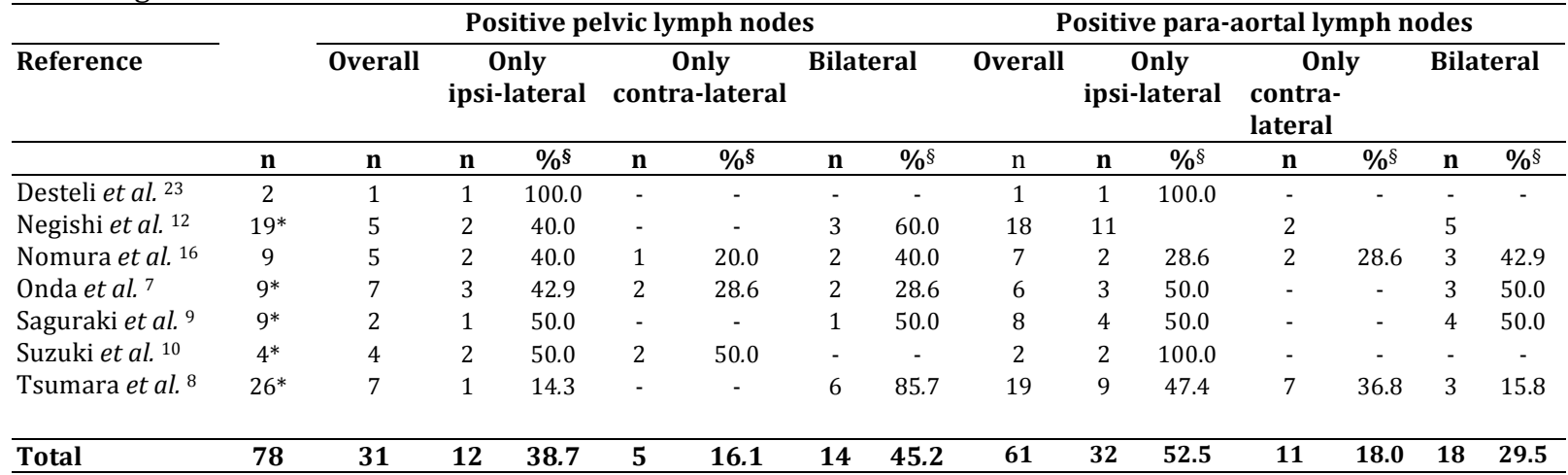

$\S$ The percentage indicates the number of patients with positive lymph nodes in that particular anatomical region as a proportion of the patients with positive lymph nodes. 


\section{Discussion}

EOC is often considered as a homogeneous disease and as such treated, without taking the anatomical localization, differentiation grade or histological subtype into account. The goal of this systematic review was to explore whether pathological variables such as differentiation grade and histological subtype can influence the incidence of lymph node metastases, and whether the laterality will influence the distribution of positive lymph nodes in the pelvis. To do so we first calculated the overall incidence of positive lymph nodes in clinically apparent FIGO stage I-II disease. The overall mean incidence of $14.2 \%$ suggests that a systematic lymphadenectomy or at least a thorough sampling should always be carried out to maximize the chance of finding positive lymph nodes. Although all studies state to have performed a complete and systematic lymphadenectomy, we noticed that three studies stated a range of removed lymph nodes starting at two. ${ }^{11,19,24}$ The completeness of such a lymphadenectomy is doubtful and the accuracy of these reports is therefore questionable, unless in these cases the lymphadenectomy was stopped based on obvious lymph node metastases.

The para-aortic route is to be considered as the main route of lymphatic spread with half of the patients having only lymph node metastases in the para-aortic region. However, restricting a lymphadenectomy to the para-aortic region is not recommended due to the fact that one fourth of the patients had only pelvic lymph node metastases. Omitting the pelvic lymphadenectomy would therefore cause an underdiagnosis of stage IIIc disease. Lymphadenectomy should also include the contra-lateral site even in patients with a macroscopical unilateral ovarian tumour, since in case of pelvic and/or parta-aortal lymph node metastases they are localized only at the contra-lateral site in $16-18 \%$ of the cases. There are several reasons why a macroscopic tumour can cause contralateral lymph node metastases. First, microscopic disease can already have spread to the other pelvic wall or ovary. This will not be noticed until after surgery when the pathologist will examine both ovaries. Second, possible there is a presacral lymphatic route through which the malignant cells can spread to the contralateral side. Therefore, we feel it is reasonable to perform a bilateral pelvic and para-aortal 
lymphadenectomy or thorough lymph node sampling even in the case of a macroscopic unilateral ovarian tumour.

There is evidence that optimal surgical staging identifies patients who have no gain from adjuvant chemotherapy, especially patients with well to moderate differentiated tumours. ${ }^{25}$ Therefore, to provide optimal management of a patient with an adnexal mass, it is important to differentiate between malignant and benign pathology prior to and during surgery. The preoperative assessment of an adnexal mass is still a diagnostic challenge. Several parameters have been described, and some authors have developed prediction models that combine patient characteristics, sonographic results, and/or serum markers. ${ }^{26,27}$ However, all prognostic models as well as clinical judgment have demonstrated to lack perfect diagnostic accuracy. An additional diagnostic procedure for the assessment of the adnexal mass during surgery is frozen section diagnosis. The accuracy of frozen section diagnosis for the assessment of the ovarian mass is good, with acceptable sensitivities and almost perfect specificities. ${ }^{28}$ Nevertheless, sometimes the diagnosis of ovarian cancer can only be made after the operation. These patients need to be restaged during a second procedure. During these restaging procedures the chances of upstaging a patient to stage III-IV is approximately $30 \% .^{29}$ As shown in this present overview, there is $14.2 \%$ chance of finding lymph node metastases, and therefore a staging procedure, including an extensive pelvic and para-aortic sampling or complete lymphadenectomy has to be performed at all times.

With regards to the differentiation grade, we found that the chance of finding positive lymph nodes in grade 1 tumours was lowest with an incidence of $4.0 \%$. With regards to histological subtypes, we noticed that the incidence of positive lymph nodes was only $2.6 \%$ in mucinous tumours. Although we were not able to discern this figure from the literature, we can presume that the incidence of positive lymph nodes is even lower when both pathological parameters are combined. The chance of finding lymph node metastases in grade I mucinous tumours is therefore slim. In this subgroup of patients it can be considered to omit a systematic lymphadenectomy when a restaging procedure is indicated due to the low incidence of positive lymph nodes and the considerable morbidity of a 
systematic pelvic and para-aortic lymphadenectomy. ${ }^{30}$ However, a limitations of this review is related to the differentiation grade and tumour histology. In none of the studies included in this review, a revision of the histology was performed. It is known that especially mucinous borderline tumours can be erroneously be seen as well differentiated mucinous ovarian cancer which may result in a falsely low percentage of lymph node metastases.

In conclusion this review shows the incidence of node metastases is considerable in clinical early-stage disease and concerns both the para-aortic and pelvic lymph nodes. The incidence of lymph nodes metastases is the highest in tumours with a poor differentiation grade and/ or serous type histology. Based on the scarce literature data, omitting a systematic lymphadenectomy might only be considered in grade I mucinous tumours. 


\section{References}

1. Epitheliaal ovarium carcinoom. 2009 [cited 2010 19-02]; Available from: http://www.oncoline.nl/index.php?pagina=/richtlijn/item/pagina.php\&id $=28908 \&$ richtlijn_id=631.

2. Cancer Facts \& Figures 2009. Estimated New Cancer Cases and Deaths by Sex, US: American Cancer Society;2009.

3. Panici PB, Angioli R. Role of lymphadenectomy in ovarian cancer. Best Pract Res Clin Obstet Gynaecol 2002;16:529-551.

4. an DS, Agarwal R, Kaye SB. Mechanisms of transcoelomic metastasis in ovarian cancer. Lancet Oncol 2006;7:925-934.

5. Burghardt E, Girardi F, Lahousen M, Tamussino K, Stettner H. Patterns of pelvic and paraaortic lymph node involvement in ovarian cancer. Gynecol Oncol 1991;40:103-106.

6. Benedetti-Panici P, Greggi S, Maneschi F, Scambia G, Amoroso M, Rabitti C, Mancuso S. Anatomical and pathological study of retroperitoneal nodes in epithelial ovarian cancer. Gynecol Oncol 1993;51:150-154.

7. Onda T, Yoshikawa H, Yokota H, Yasugi T, Taketani Y. Assessment of metastases to aortic and pelvic lymph nodes in epithelial ovarian carcinoma. A proposal for essential sites for lymph node biopsy. Cancer 1996;78:803-808.

8. Tsumura N, Sakuragi N, Hareyama H, Satoh C, Oikawa M, Yamada H, Yamamoto R, Okuyama K, Fujino T, Sawaga T, Fujimoto S. Distribution pattern and risk factors of pelvic and para-aortic lymph node metastasis in epithelial ovarian carcinoma. Int J Cancer 1998;79:526-530.

9. Sakuragi N, Yamada H, Oikawa M, Okuyama K, Fujino T, Sagawa T, Fujimoto S. Prognostic significance of lymph node metastasis and clear cell histology in ovarian carcinoma limited to the pelvis (pT1M0 and pT2M0). Gynecol Oncol 2000;79:251-255.

10. Suzuki M, Ohwada M, Yamada T, Kohno T, Sekiguchi I, Sato I. Lymph node metastasis in stage I epithelial ovarian cancer. Gynecol Oncol 2000;79:305308.

11. Morice P, Joulie F, Camatte S, Atallah D, Rouzier R, Pautier P, Pomel C, Lhommé C, Duvillard P, Castaigne D. Lymph node involvement in epithelial 
ovarian cancer: analysis of 276 pelvic and paraaortic lymphadenectomies and surgical implications. J Am Coll Surg 2003;197:198-205.

12. Negishi H, Takeda M, Fujimoto T, Todo Y, Ebina Y, Watari H, Yamamoto R, Minakami H, Sakuragi N. Lymphatic mapping and sentinel node identification as related to the primary sites of lymph node metastasis in early stage ovarian cancer. Gynecol Oncol 2004;94:161-166.

13. Takeshima N, Hirai Y, Umayahara K, Fujiwara K, Takizawa K, Hasumi K. Lymph node metastasis in ovarian cancer: difference between serous and non-serous primary tumours. Gynecol Oncol 2005;99:427-431.

14. Maggioni A, Benedetti Panici P, Dell'Anna T, Landoni F, Lissoni A, Pellegrino A, Rossi RS, Chiari S, Campagnutta E, Greggi S, Angiolo R, Manci N, Calcagno M, Scambia G, Fossati R, Floriani I, Torri, Grassi R, Mangioni C. Randomised study of systematic lymphadenectomy in patients with epithelial ovarian cancer macroscopically confined to the pelvis. Br J Cancer 2006;18:699704.

15. Harter P, Gnauert K, Hils R, Lehmann TG, Fisseler-Eckhoff A, Traut A, du Bois A. Pattern and clinical predictors of lymph node metastases in epithelial ovarian cancer. Int J Gynecol Cancer 2007;17:1238-1244.

16. Nomura H, Tsuda H, Susumu N, Fujii T, Banno K, Kataoka F, Tominaga E, Suzuki A, Chiyoda T, Aoki D. Lymph node metastasis in grossly apparent stages I and II epithelial ovarian cancer. Int J Gynecol Cancer 2010;20:341345.

17. Ang D, Ng KY, Tan HK, Chung AY, Yew BS, Lee VK. Ovarian carcinoma presenting with isolated contralateral inguinal lymph node metastasis: a case report. Ann Acad Med Singapore 2007;36:427-430.

18. Oei AL, de Hullu JA, Grefte JM, Havenith BP. An enlarged groin node as first manifestation of a malignancy: don't forget the ovaries. Eur J Obstet Gynecol Reprod Biol 2008;138:240-242.

19. Fournier M, Stoeckle E, Guyon F, Brouste V, Thomas L, MacGrogan G, Floquet A. Lymph node involvement in epithelial ovarian cancer: sites and risk factors in a series of 355 patients. Int J Gynecol Cancer 2009;19:13071313. 
20. Carnino F, Fuda G, Ciccone G, Iskra L, Guercio E, Dadone D, Conte PF. Significance of lymph node sampling in epithelial carcinoma of the ovary. Gynecol Oncol 1997;65:467-472.

21. Benedet JL, Bender H, Jones H, 3rd, Ngan HY, Pecorelli S. FIGO staging classifications and clinical practice guidelines in the management of gynecologic cancers. FIGO Committee on Gynecologic Oncology. Int J Gynaecol Obstet 2000;70:209-262.

22. Carnino F, Fuda G, Ciccone G, Iskra L, Guercio E, Dadone D, Conte PF. Significance of lymph node sampling in epithelial carcinoma of the ovary. Gynecol Oncol 1997;65:467-472.

23. Desteli GA, Gultekin M, Usubutun A, Yuce K, Ayhan A. Lymph node metastasis in grossly apparent clinical stage Ia epithelial ovarian cancer: Hacettepe experience and review of literature. Wold J of Surg Onc 2010;8:106.

24. Ayhan A, Gultekin M, Taskirana C, Celik NY, Usubutunb A, Kucukali T, Yuce K. Lymphatic metastasis in epithelial ovarian carcinoma with respect to clinicopathological variables. Gynecol Oncol 2005;95:400-404.

25. Timmers PJ, Zwinderman K, Coens C, Vergote I, Trimbos JB. Lymph node sampling and taking of blind biopsies are important elements of the surgical staging of early ovarian cancer. Int J Gynecol Cancer 2010;20:1142-1147.

26. Geomini P, Kruitwagen R, Bremer GL, Cnossen J, Mol BW. The accuracy of risk scores in predicting ovarian malignancy: a systematic review. Obstet Gynecol 2009;113:384-94.

27. Van Gorp T, Cadron I, Despierre E, Daemen A, Leunen K, Amant F, Timmerman D, De Moor B, Vergote I. HE4 and CA125 as a diagnostic test in ovarian cancer: prospective validation of the Risk of Ovarian Malignancy Algorithm. Brit J of Cancer 2011;104:863-870.

28. Geomini P, Bremer G, Kruitwagen R, Mol BW. Diagnostic accuracy of frozen section diagnosis of the adnexal mass: a metaanalysis. Gynecol Oncol 2005;96:1-9.

29. Chan JK, Munro EG, Cheung MK, Husain A, Teng NN, Berek JS, Osann K. Association of lymphadenectomy and survival in stage I ovarian cancer patients. Obstet Gynecol 2007;109:12-19. 
30. Benedetti Panici P, Maggioni A, Hacker N, Landoni F, Ackermann S, Campagnutta E, Tamussino K, Winter R, Pellegrino A, Greggi S, Angioli R, Manci N, Scambia G, Dell'Anna T, Fossati R, Florani I, Rossi RS, Grassi R, Favalli G, Raspagliesi F, Giannarelli D, Martella L, Mangioni C. Systematic aortic and pelvic lymphadenectomy versus resection of bulky nodes only in optimally debulked advanced ovarian cancer: a randomized clinical trial. J Natl Cancer Inst 2005;97:560-566. 


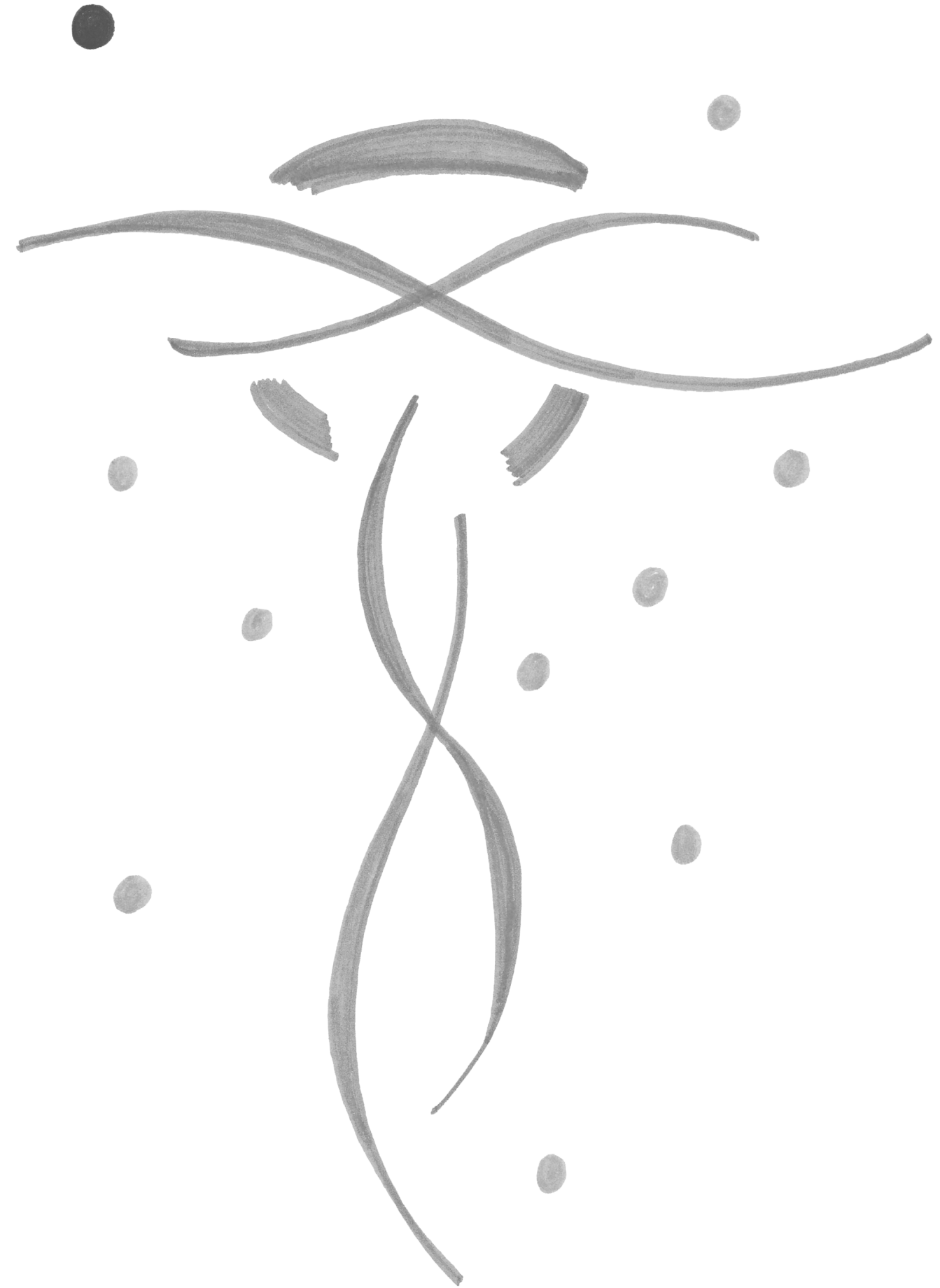




\section{Chapter 3}

\section{Lymph-node metastasis}

in stage I and II sex cord stromal and malignant

\section{germ cell tumours of the}

\section{ovary: a systematic}

review

Kleppe M, Amkreutz LCM, Van Gorp T, Slangen BFM, Kruse AJ, Kruitwagen RFPM 


\begin{abstract}
Objectives

The aim of this systematic review is to determine the incidence of lymph-node metastasis in clinical stage I and II sex cord stromal tumours and germ cell tumours of the ovary.
\end{abstract}

\title{
Methods
}

Relevant articles were identified from MEDLINE and EMBASE and supplemented with citations from the reference lists of the primary studies. Eligibility was determined by two authors. Included studies were prospective or retrospective cohort and cross-sectional studies analysing at least ten patients with clinical early-stage non-epithelial ovarian cancer who underwent lymphadenectomy or lymph-node sampling as part of a staging laparotomy.

\section{Results}

For sex cord stromal tumours, five articles including 578 patients were analysed and lymph-node metastasis was not detected in the 86 patients who underwent lymph-node removal. The median number of removed lymph nodes was 13 (range 9-29).

For malignant germ cell tumours, four articles were eligible including 2,436 patients of whom 946 patients underwent lymph-node resection. The mean number of removed nodes was 10 (range 2-14) with a mean incidence of lymphnode metastasis of $10.9 \%$ (range $10.5-11.8 \%$ ).

\section{Conclusions}

The incidence of lymph-node metastasis in patients with clinical stage I and II sex cord stromal tumours is low, whereas the incidence in patients with clinical stage I-II germ cell tumours is considerable, although limited data are available. 


\section{Introduction}

Non-epithelial ovarian cancer, which accounts for approximately $10 \%$ of all malignant ovarian tumours, is classified into malignant ovarian sex cord stromal tumours (SCSTs) and ovarian germ cell tumours (GCTs). ${ }^{1}$ SCSTs have a good longterm prognosis. The average age at diagnosis of adult granulosa cell tumours is $\mathbf{5 0}$ years. Juvenile granulosa cell tumours can present at a younger age but account for only $5 \%$ of cases. Sertoli-Leydig cell tumours usually occur under the age of 40. The yearly-adjusted incidence rate is 3.7/100.000 for GCT and 2.1/100.000 for SCST. ${ }^{2}$ GCTs are rapidly growing, predominantly unilateral tumours. They are mostly found in girls and young women and cure rates are high. Therefore, a fertility-sparing approach is often considered. ${ }^{3}$

In clinical early-stage non-epithelial ovarian cancer, the recommended surgical treatment is similar to the surgical staging procedure in epithelial ovarian cancer. As defined by the International Federation of Gynaecology and Obstetrics (FIGO), this includes a systematic pelvic and para-aortic lymphadenectomy. ${ }^{2}$ Patients with lymph node metastases are classified as FIGO stage IIIC disease, even in the absence of peritoneal metastases. Omitting a lymphadenectomy could cause underdiagnosis of stage IIIC disease, leading to denial of adjuvant chemotherapy with a curative intention. Despite these guidelines, many patients with nonepithelial ovarian cancer are not completely staged. Lymphadenectomy or lymphnode sampling may not be performed for two reasons. Firstly, a general gynaecologist might not be capable of performing this procedure. Secondly, the diagnosis is often made after an operation for a presumed benign ovarian mass and in these cases, whether or not to perform a second operation is questionable, especially when the incidence of lymph node metastases appears to be very rare. ${ }^{4}$

In this review we aimed to identify the incidence of lymph-node metastasis in clinical stage I and II SCST and GCT. 


\section{Patients and methods}

Search strategy and selection criteria

A literature search was performed on MEDLINE and EMBASE. Specific searches were performed with the terms "non-epithelial ovarian cancer", "lymph nodes", "spread”, "metastasis”, "lymphadenectomy”, “sex cord stromal tumour”, "granulosa cell tumour", "germ cell tumour" and "ovary". Separate searches were performed with MeSH terms and clinical queries on MEDLINE to extract all available relevant literature. The searches were limited to "English" and "human". Additional literature was searched through cross-referencing using the retrieved articles. The inclusion criteria were as follows: (a) prospective and retrospective cohort or cross-sectional studies, and (b) studies that included at least 10 patients with clinical FIGO stage I or II SCST or GCT or subtypes of these tumours who underwent lymphadenectomy or lymph-node sampling.

\section{Data extraction and quality assessment}

Two authors (MK and LCMA) independently evaluated the abstracts of selected studies to determine eligibility. The full text was evaluated if the abstract did not provide sufficient information for the determination of eligibility. A standard data extraction form was used to collect data from eligible publications. Data extraction was conducted by two authors (MK and LCMA), and discrepancies were resolved by discussion with a third co-author (RFPMK). If key aspects of the studies did not provide sufficient information, the authors were contacted by email and asked to provide additional information. Data extraction included the following variables: study type, total number of included patients, number of FIGO stage I-II patients, number of FIGO stage I-II patients with removed lymph nodes, number of removed lymph nodes (mean, median, range), extent of lymph-node dissection (pelvic and/or para-aortic region), and number of patients with positive lymph nodes. When possible, data extraction was performed for FIGO stage I and FIGO stage II separately.

\section{Results}

Study selection and characteristics 
Of 112 citations, 10 articles which met the inclusion criteria based on the title and abstract were identified. After review of the full-length articles, an additional four studies that did not meet the inclusion criteria $(n=2)$ or missed essential information $(n=2)$ were excluded, and the authors were contacted. However, we were not able to obtain additional information. An additional three studies were included through cross-referencing that were not identified in the original literature search. Finally, nine studies were included, all of which were retrospective cohort studies. ${ }^{3,4,5-10}$ In six articles information was missing. The number of patients with stage I-II disease who underwent lymphadenectomy was not specified ${ }^{5,8}$, and the total number of stage I-II patients was not mentioned. ${ }^{9}$ In three studies, the number of removed nodes (mean, median, range) was not mentioned.6,9,10

\section{Sex cord stromal cell tumours}

Five studies analysing lymph-node involvement in SCST were included (Table 1).4, 5-8 One study only described adult granulosa cell tumours. ${ }^{7}$ In studies that included other subtypes, the majority of the patients had an adult granulosa cell tumour.

Complete pelvic and para-aortic lymphadenectomy was obligatory in one study. ${ }^{6}$

Table 1: Incidence of positive lymph nodes in FIGO stage I-II sex cord stromal cell tumours.

\begin{tabular}{|c|c|c|c|c|c|c|c|c|}
\hline \multirow[t]{2}{*}{ Reference } & Patients & $\begin{array}{l}\text { FIGO } \\
\text { stage } \\
\text { I + II }\end{array}$ & $\begin{array}{c}\text { FIGO } \\
\text { stage I + } \\
\text { II with } \\
\text { removed }\end{array}$ & \multicolumn{4}{|c|}{$\begin{array}{c}\text { Number of removed lymph nodes per } \\
\text { patient }\end{array}$} & \multirow{2}{*}{$\begin{array}{c}\text { Patients with } \\
\text { positive } \\
\text { lymph nodes }\end{array}$} \\
\hline & $\mathbf{n}$ & $\mathbf{n}$ & $\mathbf{n}$ & Locations & Mean & Median & Range & \\
\hline Abu-Rustum et al. ${ }^{5}$ & 68 & 60 & NS & $\begin{array}{c}\text { Pelvic } \\
\text { Para-aortic }\end{array}$ & $\begin{array}{c}11.6 \\
6\end{array}$ & $\begin{array}{c}10 \\
4\end{array}$ & $\begin{array}{l}0-36 \\
0-19\end{array}$ & 0 \\
\hline Ayhan et al. ${ }^{6}$ & 60 & 38 & 12 & - & - & - & - & 0 \\
\hline Brown et al. ${ }^{4}$ & 257 & 86 & 49 & $\begin{array}{c}\text { Pelvic } \\
\text { and/or } \\
\text { para-aortic }\end{array}$ & - & 9 & $3-28$ & 0 \\
\hline Park et al. ${ }^{7}$ & $106^{\#}$ & 93 & 25 & $\begin{array}{c}\text { Pelvic } \\
\text { and/or } \\
\text { para-aortic }\end{array}$ & 28.8 & 29 & $5-53$ & 0 \\
\hline Thrall et al. ${ }^{8}$ & 87 & 56 & NS & $\begin{array}{c}\text { Pelvic and } \\
\text { para-aortic }\end{array}$ & - & 14 & $3-80$ & 0 \\
\hline Total & 578 & 333 & - & - & - & - & & $\mathbf{0}$ \\
\hline
\end{tabular}

Abbreviations: FIGO: International Federation of Obstrectics and Gynecology, NS: not specified. 
Detection of lymph-node metastasis was not reported in any of the five studies. ${ }^{4,5-8}$ Two studies did not report the number of patients with clinical stages I and II who underwent lymph-node removal.5, 8 In the three studies that specified the number of patients with removed lymph nodes, no positive lymph nodes were detected in 86 patients. ${ }^{4,6,7}$

\section{Germ cell tumours}

Three studies on GCT with a total of 2,436 patients were included in the analysis (Table 2).3, 9, 10 Complete pelvic and para-aortic lymphadenectomy was obligatory in one study. ${ }^{10}$ In the analysis of lymph-node metastasis, 946 out of 2,436 patients had stage I-II GCT and underwent lymph-node dissection, with positive lymph nodes detected in 103 patients (10.9\%, range $0-11.8 \%)$.

Table 2. Incidence of positive lymph nodes in FIGO stage I-II malignant germ cell tumours.

\begin{tabular}{|c|c|c|c|c|c|c|c|c|}
\hline \multirow{2}{*}{ Reference } & \multirow{2}{*}{$\begin{array}{c}\text { Patients } \\
\mathrm{n}\end{array}$} & \multirow{2}{*}{$\begin{array}{c}\text { FIGO } \\
\text { stage } \\
\text { I + II } \\
\mathrm{n}\end{array}$} & \multirow{2}{*}{$\begin{array}{c}\text { FIGo } \\
\text { stage I + } \\
\text { II with } \\
\text { removed } \\
\text { lymph } \\
\text { nodes }\end{array}$} & \multicolumn{4}{|c|}{$\begin{array}{c}\text { Number of removed lymph nodes } \\
\text { per patient }\end{array}$} & \multirow{2}{*}{$\begin{array}{c}\text { Patients } \\
\text { with } \\
\text { positive } \\
\text { lymph } \\
\text { nodes } \\
\text { n }(\%)\end{array}$} \\
\hline & & & & Locations & Mean & Median & Range & \\
\hline \multirow{3}{*}{ Kumar et al. ${ }^{9}$} & \multirow{3}{*}{1296} & \multirow{3}{*}{ NS } & \multirow{3}{*}{432} & - & $12^{*}$ & - & $1-96^{*}$ & \multirow{3}{*}{51 (11.8) } \\
\hline & & & & & & & & \\
\hline & & & & - & $14^{\#}$ & - & $1-96^{\#}$ & \\
\hline \multirow{3}{*}{ Madhi et al. ${ }^{3}$} & \multirow{3}{*}{1083} & \multirow{3}{*}{1083} & \multirow{3}{*}{493} & - & $11^{*}$ & $8^{*}$ & $1-47^{*}$ & \multirow{3}{*}{$52(10.5)$} \\
\hline & & & & & & & & \\
\hline & & & & - & $2^{\#}$ & $1^{\#}$ & $1-15^{\#}$ & \\
\hline Rogers et al. 10 & 57 & 57 & 21 & $\begin{array}{l}\text { Pelvic/pa } \\
\text { ra-aortic; } \\
\text { Perirenal } \\
\text { chains }\end{array}$ & - & - & - & 0 \\
\hline Total & 2436 & - & 946 & - & - & - & - & $\begin{array}{c}103 \\
(10.9 \%)\end{array}$ \\
\hline
\end{tabular}

Abbreviations: FIGO: International Federation of Obstrectics and Gynecology, NS: not specified.

*: Cohort of patients with negative nodes

\#: Cohort of patients with positive nodes 
In one article, the incidence of lymph-node metastasis was determined according to histological subtype. ${ }^{3}$ The incidence rates of nodal metastasis in patients with dysgerminoma, malignant teratoma, and not otherwise specified GCT were $18.1 \%$ (40/221), 3.9\% (7/178), and 5.3\% (5/94), respectively.

\section{Discussion}

The present systematic review showed that lymph-node metastases were absent in clinical stage I-II SCST, whereas the incidence of lymph-node metastasis in stage I-II GCT was approximately $11 \%$, with the highest incidence in patients with dysgerminoma of the ovary (18.1\%).

The surgical approach varied widely in the different studies and a complete staging procedure, as advised by FIGO (hysterectomy with bilateral salpingooophorectomy and infracolic omentectomy, biopsy of the diaphragmatic peritoneum, paracolic gutters, pelvic peritoneum and peritoneal washings, and a systematic pelvic and para-aortic lymphadenectomy), was performed in a small percentage of patients. ${ }^{2}$ There are several possible explanations for this. Firstly, tumours are often assumed to be benign before and during surgery. Once the pathological diagnosis is made, a relaparotomy to complete the surgical staging is not considered obligatory. Secondly, fertility-sparing surgery is often preferred, in particular in patients of reproductive age. Thirdly, patients with GCT may include young children and adolescents, and whether an extensive surgical staging procedure should be offered to these young patients remains questionable. Therefore, physicians often avoid extensive staging procedures because of the high sensitivity of GCT for chemotherapy. The current platinum-based chemotherapy regimes used in children are associated with excellent survival rates (93.3-98.3\%) for stage I-IV GCT. Survival is unaffected by deviations from the standard surgical approach $^{11}$, although lymph-node involvement is an independent predictor of a less favourable survival in these patients. ${ }^{9}$ The removal of lymph nodes is therefore questionable for staging purposes.

The mean number of lymph nodes removed in SCST was only mentioned in two out of five studies. ${ }^{4-8}$ In both studies an adequate number of removed lymph nodes 
(18 and 28.8) was reported. However studies on epithelial ovarian cancer have suggested a positive correlation between the number of lymph nodes removed and the detection of nodal metastasis. ${ }^{12}$ Nevertheless, lymph-node metastasis was not detected in patients with clinical stage I-II SCST. Lymphadenectomy is a radical procedure associated with a higher morbidity than lymph-node sampling. Among the complications of lymphadenectomy, the formation of lymphocysts (up to $13.5 \%$ ), nerve and vessel injury (up to $4 \%$ ), increased blood loss and increased operating time have been reported. ${ }^{13}$ Omitting a lymphadenectomy or lymph-node sampling in a known clinical early-stage SCST could therefore decrease costs and morbidity without decreasing survival.

The incidence of lymph-node metastasis in GCT was approximately $11 \%$. Patients with dysgerminoma of the ovary showed the highest incidence of lymph-node metastasis (18.1\%), as described in one study that included information on the incidence of lymph-node metastasis from histology findings. ${ }^{3}$ This suggests that a dysgerminoma is associated with a high risk of lymph-node metastasis. However, a definite conclusion cannot be made on the basis of the limited number of studies available.

Of the nine studies that examined SCST or GCT, six reported the removal of paraaortic and pelvic lymph nodes, whereas the location of lymph-node removal was not mentioned in the remaining three studies. Previous studies on epithelial ovarian cancer reported that lymph-node metastases are mostly para-aortic; however, they also occur in the pelvic area, in both the ipsilateral and contralateral sides. $^{13}$ The location of positive lymph nodes was not specified in any of the studies. Therefore, a preferred location of metastasis cannot be defined on the basis of the available literature on SCST and GCT. The removal of para-aortic and pelvic lymph nodes, including those ipsilateral and contralateral to the tumour, is recommended during lymph-node sampling in SCST and GCT based on the characteristics of ovarian cancer.

This systemic review had several limitations. Firstly, the number of published studies on lymph-node involvement in non-epithelial ovarian cancer is limited 
because of the low incidence of this disease. Secondly, because the rate of adherence to the staging procedure suggested by FIGO is low, the total number of stage I or II patients who underwent lymph-node removal was also low.

Nevertheless, based on the available evidence, we can conclude that the incidence of lymph-node metastasis in patients with SCST is low, whereas the incidence of lymph-node metastasis in patients with GCT is considerable (+/- 10\%). Despite the lack of accurate prospective data, our review of the literature suggests that the need for lymphadenectomy or lymph-node sampling in SCST should be reassessed because of the low rate of lymph node metastases associated with this tumour type. The situation in patients with a GCT is more complex because the rate of lymph-node metastases is high. However, even in patients with advanced stage GCT, the prognosis is excellent because of the enhanced chemosensitivity of this tumour. In a recent retrospective multicentre study no significant difference in overall survival was found between patients with GCT obtaining fertility preserving (ipsilateral adnexectomy) comprehensive staging surgery (including retroperitoneal lymphadenectomy) compared to patients in whom an ipsilateral adenexectomy was only combined with a clinical intraoperative evaluation (only biopsies or excisions of suspicious sites). The survival rates at 5 years were $92 \%$ and $97 \%$, respectively. ${ }^{14}$ However, nearly all patients (96\%) received adjuvant chemotherapy in this study population. It remains to be determined whether the same overall survival can be achieved when adjuvant chemotherapy is not given in an adjuvant setting in incompletely staged early-stage disease and postponed until a lymphogenic recurrence becomes apparent. Based on the available evidence, we advise re-operating an incompletely staged patient with a germ cell tumour, at least when no adjuvant chemotherapy is considered. There is no need for reintervention when adjuvant chemotherapy is considered anyway. 


\section{References}

1. Gershenson DM. Current advances in the management of malignant germ cell and sex cord-stromal tumours of the ovary. Gynecol Oncol 2012;125:515-517.

2. Colombo N, Peiretti M, Garbi A, Carinelli S, Marini C, Sessa C. Non-epithelial ovarian cancer: ESMO clinical practice guidelines for diagnosis, treatment and follow-up. Ann Oncol 2012;23:20-26.

3. Madhi H, Swensen RE, Hanna E, Kumar S, Ali-Fehmi R, Tamimi H, Morris RT, Munkarah AR. Prognostic impact of lymphadenectomy in clinical early stage malignant germ cell tumour of the ovary. British Journal of Cancer 2011;105:493-497.

4. Brown J, Sood AK, Deavers MT, Milojevic L, Gershenson DM. Patterns of metastasis in sex cord-stromal tumours of the ovary: Can routine staging lymphadenectomy be omitted? Gynecol Oncol 2009;113:86-90.

5. Abu-Rustum NR, Restivo A, Ivy J, Soslow R, Sabbatini P, Sonoda Y Barakat $\mathrm{RR}$, Chi DS. Retroperitoneal nodal metastasis in primary and recurrent granulosa cell tumours of the ovary. Gynecol Oncol 2006;103:31-34.

6. Ayhan A, Tuncer ZS, Tuncer R, Mercan R, Yüce K, Ayhan A. Granulosa cell tumour of the ovary. A clinicopathological evaluation of 60 cases. Eur J Gynaecol Oncol 1994;15:320-324.

7. Park JY, Jin KL, Kim DY, Kim JH, Kim YM, Kim KR, Kim YT, Nam JH. Surgical staging and adjuvant chemotherapy in the management of patients with adult granulosa cell tumours of the ovary. Gynecol Oncol 2012;125:80-86.

8. Thrall MM, Paley P, Pizer E, Garcia R, Goff B. Patterns of spread and recurrence of sex cord-stromal tumours of the ovary. Gynecol Oncol 2011;122:242-245.

9. Kumar S, Shah JP, Bryant CS, Imudia AN, Cote ML Ali-Fehmi R, Malone JM Jr, Morris RT. The prevalence and prognostic impact of lymph node metastasis in malignant germ cell tumours of the ovary. Gynecol Oncol 2008;110:125132.

10. Rogers PC, Olson TA, Cullen JW, Billmire DF, Marina N. Treatment of children and adolescents with stage II testicular and stages I and II ovarian malignant germ cell tumours: a pediatric intergroup study- Pediatric 
oncology group 9048 and children's cancer group 8891. J Clin Oncol 2004;22:3563-3569.

11. Billmire D, Vinocour C, Rescorla F, Cushing B, London W, Schlatter M, Davis M, Giller R, Lauer S, Olson T; Children's Oncology Group (COG). Outcome and staging evaluation in malignant germ cell tumours of the ovary in children and adolescents: an intergroup study. J Pediatr Surg 2004;39:424429.

12. Kleppe M, Wang T, Van Gorp T, Slangen BFM, Krüse AJ, Kruitwagen RFPM. Lymph node metastasis in stages I and II ovarian cancer: a review. Gynecol Oncol 2011;123:610-614.

13. Di Re F, Baiochocci G. Value of lymph node assessment in ovarian cancer: status of the art at the end of the second millennium. Int J Gynecol Cancer 2000;10:435-442.

14. Liu Q, Ding X, Yang J, Cao D, Shen K, Lang J, Zhang G, Xin X, Xie X, Wu Y. The significance of comprehensive staging surgery in malignant ovarian germ cell tumors. Gynecol Oncol 2013;131:551-554. 


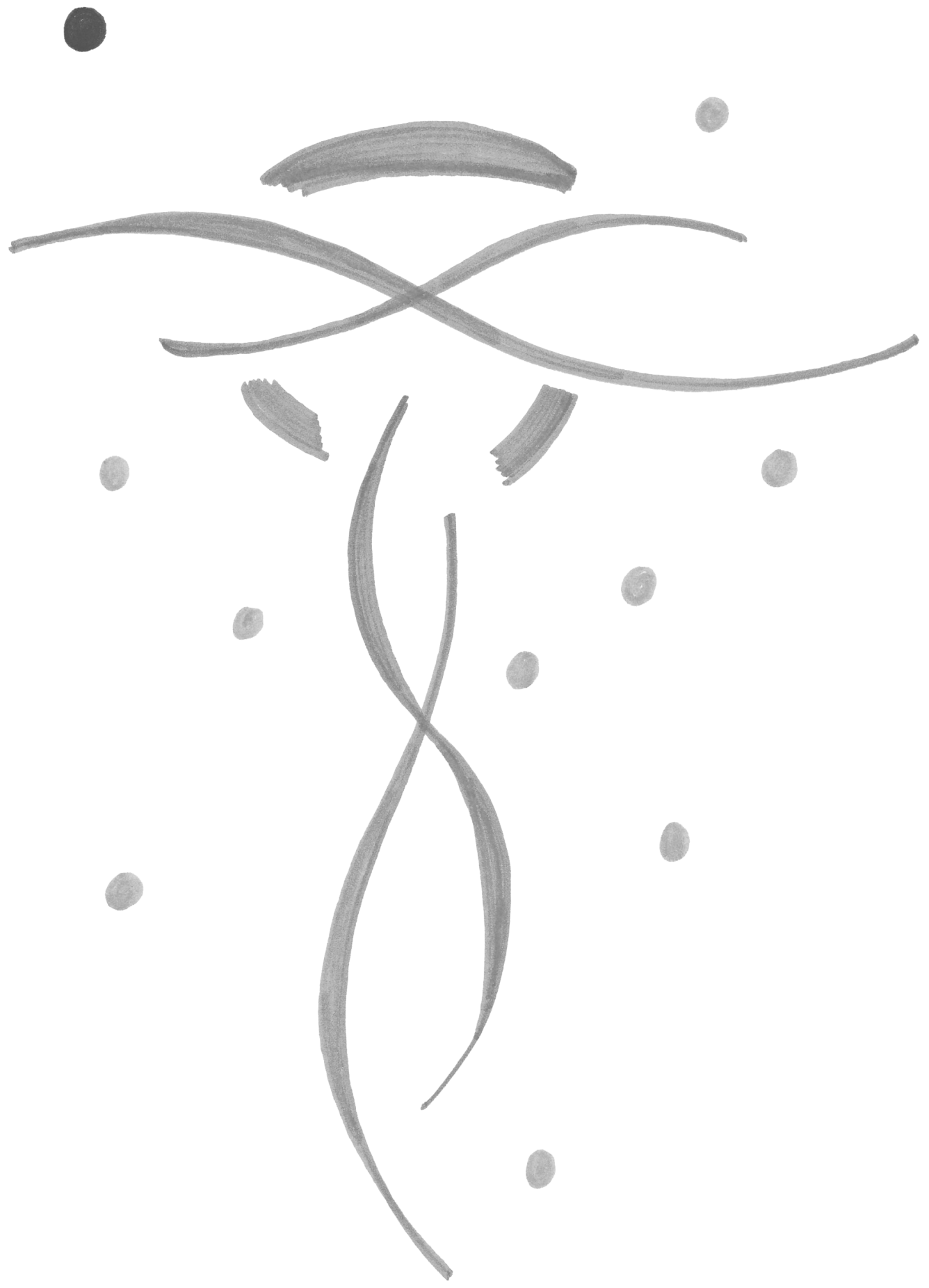




\section{Chapter 4}

\section{Sentinel node in ovarian}

\section{cancer: study protocol}

\section{for a phase 1 study}

Kleppe M, Van Gorp T, Slangen BFM, Kruse AJ, Brans B, Pooters INA, Van de Vijver KK, Kruitwagen RFPM

Trials 2013;14:47 


\section{Abstract}

\section{Background}

The concept of sentinel lymph node surgery is to determine whether the cancer has spread to the very first lymph node or sentinel node. If the sentinel node does not contain cancer, then there is a high likelihood that the cancer has not spread to other lymph nodes. The sentinel node technique has been proven to be effective in different types of cancer. In this study we want to determine whether a sentinel node procedure in patients with ovarian cancer is feasible when the tracers are injected in the ovarian ligaments.

\section{Methods/design}

Patients with a high suspicion of an ovarian malignancy in whom a median laparotomy and a frozen section analysis is planned and patients with endometrial cancer in whom a staging laparotomy is planned will be included.

Before starting the surgical staging procedure, blue dye and radioactive-colloid will be injected in the ligamentum ovarii proprium and the ligamentum infundibulo-pelvicum. In the analysis we calculate the percentage of patients in whom it is feasible to identify sentinel nodes. Other study parameters are the anatomical localisation of the sentinel node(s) and the incidence of false negative lymph nodes.

\section{Trial registration}

Approval number: NL40323.068.12

Name: Medical Ethical Committee Maastricht University Hospital, University of Maastricht

Affiliation: Maastricht University Hospital

Board Chair Name: Medisch Ethische Commissie azM/UM 


\section{Background}

Epithelial ovarian cancer (EOC) remains the tumour with the most unfavourable prognosis within the field of the gynaecological oncology. The incidence of ovarian cancer in the Netherlands in 2008 was 14.5 per 100.000 , with 12.3 deaths per 100.000. ${ }^{1}$ In the US in 2007 the incidence was 13.0 per 100.000 and there were 8.2 deaths per $100.000 .^{2}$ The high mortality rate is partially due to the fact that approximately $75 \%$ of patients are diagnosed with advanced stage EOC.

EOC can metastasize through three different ways: intraperitoneal (in the abdominal cavity), lymphogenous and haematogenous. ${ }^{3,4}$ Concerning the lymphogenous spread, it is clear that lymphatic metastases of EOC mainly occur in the para-aortic lymph nodes. ${ }^{5}$ It is believed that the tumour cells follow the lymph vessels that accompany the ovarian artery and vein in the infundibulopelvic ligament up to the high para-aortic region and renal vein. Nevertheless, pelvic lymph node metastases are also frequently found.6-16 These tumour cells probably follow a different route, following the para-uterine vessels in the broad ligament towards the uterine artery and vein and further on to the iliac vessels. In some case reports isolated inguinal node metastases are also described. ${ }^{17-20}$ The exact mechanism of this route of metastasis is still unclear, but the metastatic cells might follow the course of the round ligament towards the inguinal lymph nodes, or might follow the iliac vessels towards the femoral vessels. The incidence of lymph node metastasis in clinical stage I-II ovarian carcinoma is $14.2 \% .^{21}$

In case of a clinical early-stage ovarian cancer, the Dutch guideline recommends a staging laparotomy with adequate lymph node sampling, with an absolute minimum of ten lymph nodes removed. ${ }^{1}$ In the same guideline, a footnote is made stating that a larger number of removed lymph nodes will increase the chance of finding metastases. These lymph nodes also need to be sampled from different anatomical regions, of which the most important are the para-aortic and paracaval region between the renal vein and inferior mesenteric artery, the common, internal and external iliac vessels and the obturator fossa. 
According to the International Federation of Gynecology and Obstetrics (FIGO), EOC with lymph node metastases is classified as FIGO stage IIIC disease, even in the absence of peritoneal metastases. ${ }^{22}$ In contrast to patients with FIGO stage I ovarian cancer after a comprehensive staging procedure, patients with a FIGO stage III ovarian cancer obligatory receive adjuvant chemotherapy. Therefore, the recognition of lymph node metastases is of utmost importance.

Surgical staging of EOC and the extent of lymph node dissection differs greatly from centre to centre. ${ }^{23}$

A recent review, published in 2011, showed an incidence of $14.2 \%$ for lymph node metastasis in early EOC. ${ }^{21}$ The incidence is higher in grade 3 tumours (20.0\%) and the serous histological subtype (23.3\%), whereas in grade 1 and mucinous tumours this is respectively $4.0 \%$ and $2.6 \%$.

The assessment of lymph nodes with the aid of radiological techniques (CT scan, MRI, PET) alone in EOC is insufficient: the sensitivity and specificity for detection of lymph node metastases with PET scan is $73.2 \%$ and $96.7 \%$, with CT scan $42.6 \%$ and $95.0 \%$, and with MRI $54.7 \%$ and $88.3 \%$, respectively. ${ }^{24}$

To discover the involved lymph nodes different surgical approaches exist, ranging from taking random lymph nodes in different anatomical regions (lymph node sampling) to a systematic lymphadenectomy. ${ }^{23}$ A systematic lymphadenectomy can be considered as the gold standard. However, such a radical procedure gives more morbidity than lymph node sampling. These include the formation of lymphocysts (up to 13.5\%), nerve and vessel injury (up to $4 \%$ ), increased blood loss and increased operating time. ${ }^{25}$

With a sentinel node procedure the first node that receives primary lymphatic flow can be identified (the so called sentinel node). The pathological examination is an indication of the nodal status of the remaining nodes: when the sentinel node is negative, one can presume that the remaining nodes are also not involved. As a consequence, the patient may be spared a radical lymphadenectomy, and thus the associated morbidity. 26

The sentinel node technique has been proven effective in different cancers such as breast cancer and malignant melanoma. In gynaecological tumours it has been 
shown to be effective in vulvar cancer. ${ }^{27}$ Currently, sentinel node studies are being performed for cervical en uterine cancer, and some cancer centres already perform sentinel node procedures in these gynaecological cancers routinely. Sentinel node studies in ovarian cancer are scarce. Nyberg et al performed a study in 16 patients with high-risk uterine cancer in whom technetium and blue dye were injected in the right or left ovary. ${ }^{28}$ Since patients with a high-risk uterine cancer undergo a similar staging procedure as early-stage ovarian cancer, a total abdominal hysterectomy (TAH) with bilateral salpingo-oophorectomy (BSO) and a pelvic and para-aortic lymphadenectomy, these patients were selected to investigate whether injecting tracers in the ovary would render sentinel nodes. After an incubation time of 15 minutes, a sentinel node was detected in 15 out of 16 patients. Negishi et al. used activated charcoal solution to identify ovarian lymphatics in 11 patients. ${ }^{12}$ The charcoal was injected in the cortex of the ovary. The charcoal was deposited in the lymph nodes of all patients. In both studies the tracer was injected in the ovary. Some authors claim that injecting in the ovary can be difficult when bulky ovarian masses are present. Furthermore, it is claimed that there is a risk of tumour dissemination when tracers are injected in the ovarian capsule. ${ }^{27}$ In the current feasibility study, the injection of the tracer is performed in the ovarian ligaments, not in the ovarian cortex. This is to avoid spillage and to be as close as possible to the draining lymph vessels in the ovarian ligaments, irrespective of the size of the ovarian masses. Therefore, besides patients with high-risk endometrial cancer, we also can include patients with an enlarged ovary without risk of tumour dissemination.

Lymphatic mapping can be performed with blue dyes as well as radioactive isotopes; both can be injected in the ovarian ligaments, which contain the main routes of lymph drainage. After the incubation time the sentinel nodes can be visualised by either colorization (blue lymph nodes can be identified) and/or with a gamma probe that detects the radioactive tracer. ${ }^{28}$ In breast cancer it has been shown that the detection rate is the highest when both radioactive isotope and blue dye are combined. ${ }^{29,} 30$ 
The blue dyes can cause an allergic reaction, exhibited with urticaria, erythema, hypotension and even cardiovascular collapse with bronchospasm. ${ }^{26}$ However, the incidence of allergic reactions is very low and varies between 0.07 and $2.7 \% .^{31-33}$ The radioactive isotope is safe for patient and health care workers. ${ }^{34}$ No allergic reactions have been described due to the radio-active isotope.

Since lymph node metastases are found in $14.2 \%$ of patients with clinical earlystage ovarian cancer, this is a clinically relevant study. Indeed, a sentinel node procedure can prevent unnecessary radical lymph node dissection with the associated morbidity. It could also be more accurate than at random lymph node sampling which is the current standard care in ovarian cancer in the Netherlands. This study is scientifically relevant since injecting tracers in the ovarian ligaments can enhance our knowledge on the lymphatic routes and dissemination sites of ovarian cancer, i.e. the anatomical locations where to find the lymph nodes most likely to be involved in ovarian cancer. Injection of tracer in the ovarian ligaments has, to our knowledge, never been published.

\section{Methods and analysis}

The primary objective is to determine whether or not a sentinel node procedure in patients with ovarian cancer is feasible by injecting the tracers into the ovarian ligaments instead of into the ovary itself. The secondary objectives are the anatomical location(s) and number of the sentinel node(s): detected peroperatively with blue dye, detected preoperatively with a gamma probe, and the detection of residual lymph nodes 24 hours after surgery with scintigraphy

In this feasibility study we will include both patients with (suspicion of) ovarian cancer as well as patients with a high-grade uterine carcinoma. The latter group of patients can also be included because these patients undergo the same surgical procedure; TAH with BSO and a pelvic and para-aortic lymphadenectomy or lymph node sampling.

The study will end when 20 evaluable patients are included, of which at least 10 patients with ovarian cancer. The expected inclusion period is estimated to be two 
years. If the detection rate of the sentinel node is less than $50 \%$, or when less 5 patients are included in a year, the study will be ended prematurely.

The study will be performed in the Maastricht University Medical Centre. Patients with a high suspicion of a malignant ovarian tumour planned for exploratory laparotomy will be asked to participate in the study. Only when a malignancy is confirmed on frozen section, the sentinel nodes will be removed prior to proceeding with a complete staging procedure. Patients with endometrial cancer in whom a staging laparotomy is planned are also asked to participate.

\section{Inclusion criteria:}

- Patients with a high suspicion of a malignant ovarian tumour planned for exploratory laparotomy.

- Patients with high-grade endometrial cancer in whom a staging laparotomy is planned.

- Age between 18 and 85 years.

\section{Exclusion criteria:}

- Previous surgery on one or both ovaries.

- Previous vascular surgery of the aorta, caval vein, and/or iliac vessels.

- Previous lymphadenectomy of lymph node sampling in the iliac or paraaortic region.

- History of a malignant lymphoma.

- History of a malignant tumour in the abdominal cavity.

- Previous allergic reaction to blue dye or human albumin.

- Pregnant or lactating patients.

\section{Sample size calculation:}

Between 24 and 28 patients will be included in the study.

The sample size calculation is based on the fact that:

- with a group of 20 evaluable patients the study group is large enough to give an answer whether sentinel node(s) can be detected related to the ovarian lymphatic flow when the tracers are injected in the ovarian ligaments. For this 
purpose both patients with an ovarian and endometrial malignancy, planned for a staging laparotomy can and will be included.

- patients with a benign result on frozen section of the suspicious ovary will be excluded from the study because no lymph node(s) will be resected in these patients. These patients will also be documented and described in the report.

- based on retrospective data, in $60 \%$ of the patients with a suspicious ovary, frozen section will confirm a ovarian malignancy.

- to calculate one of the secondary endpoints (incidence of false negative sentinel lymph node) at least 10 patients with ovarian cancer should be part of the 20 evaluable patients.

\section{Surgical procedure}

Patients with a high suspicion of a malignant ovarian tumour

After making the median incision and opening the abdomen, before starting with removal of the enlarged and suspicious adnex, blue dye and the radioactive isotope will be injected in the ligamentum ovarii proprium (median side) and the ligamentum infundibulo-pelvicum (lateral side), close to the ovary and just below the peritoneum. Therefore an injection with blue dye of each $0,5 \mathrm{~mL}$ will be given ventral and dorsal in the ligamentum ovarii proprium and the ligamentum infundibulo-pelvicum (total amount 2,0 mL). The same will be done with the radioactive isotope, each injection of $0,5 \mathrm{~mL}$ containing a dose of $20-\mathrm{MBq}$ technetium-99-m-labeled albumin nanocolloid (99mTc-nanocolloid or Nanocoll ${ }^{\circledR}$, GE Healthcar, Eindhoven, The Netherlands). A 15-minute interval will be planned after injection. The exact time interval for detection of positive nodes is unclear. Common practice is to wait for 15 minutes, if in this time period the node is not detected, it is considered negative. In daily practice a wait of more than 15 minutes during surgery is not feasible. At 5 and 10 minutes the radioactivity will be measured along the lymphatic tract to document whether or not perhaps sentinel node(s) can be identified at a shorter time-interval in future studies. A gamma probe measures the radioactivity. The gamma probe will be the same device in all surgical procedures.

After the 15 minutes time-interval the ovarian mass will be removed and presented to the pathologist for a frozen section. If the result is benign, no further 
actions will be performed in these patients. If the result is malignant, the sentinel node(s) will be identified either (once more) by the radioactive tracer and / or visually (blue dye) after opening the retroperitoneal space. After removal of the sentinel node(s) a complete standard staging procedure will be performed including a comprehensive sampling of other lymph nodes at the different locations.

\section{Patients with endometrial cancer in whom a staging laparotomy is planned} After making the median incision and opening the abdomen, blue dye and the radioactive isotope will be injected in the ligamentum ovarii proprium (median side) and the ligamentum infundibulo-pelvicum (lateral side) of one of the ovaries, in the same manner as decribed above in patients with a high suspicion of an ovarian malignancy. The choice of the ovary (left or right side) will alter between left and right by each patient who is included in the study.

A 15-minute interval will be planned after injection. In this time period at 5 and 10 minutes the radioactivity will be measured along the lymphatic tract to document whether or not perhaps sentinel node(s) can be identified at a shorter timeinterval in future studies. The radioactivity is measured by a gamma probe. The gamma probe will be the same device in all surgeries.

After the 15 minutes time-interval the surgical staging procedure starts with a TAH and BSO. After approximately 45 minutes the sentinel node(s) will be identified either (once more) by the radioactive tracer and / or visually (blue dye) after opening the retroperitoneal space. This 45 minutes time-interval is chosen to mimic the time interval when a frozen section is performed in case of an ovarian tumour. After removal of the sentinel node(s) a complete standard staging procedure will be performed including a comprehensive at random sampling of other lymph nodes at the different locations.

\section{After surgery}

Depending on the mobility of the patient, a scintigram will be preformed 24 hours after surgery at the nuclear department. This scintigram is performed to detect any residual radioactive hot spots. If there is any residual radioactivity this may indicate that sentinel nodes have not been identified during surgery and this 
therefore gives an indication of the reliability of identifying sentinel nodes during surgery.

\section{Data collection}

Patient characteristics. The following information will recorded:

- Age

- Surgical findings: tumour side, injection site, time between injection and detection, the number of detected sentinel nodes, anatomical location of sentinel nodes, intensity (gamma counts) of radioactive nodes, complications during surgery, side effects during surgery.

- Histology: results of the frozen section, tumour type (ovarian or endometrial), differentiation grade, histological results of sentinel and non-sentinel nodes.

- $\quad$ FIGO stage

- Postoperative scintigram, residual nodes, location of residual nodes.

The surgeon has to register the number and location of sentinel nodes resected. For this purpose the surgeon has to draw the location of the lymph nodes in an anatomical drawing (figure 1). Ten different locations will be used to report where the sentinel nodes were found:

1. High para-aortic (left side of the aorta between left renal vein and lower mesenteric artery)

2. Low para-aortic (left side of the aorta below the lower mesenteric artery)

3. High interaortacaval (between aorta and caval vein and between left renal vein and lower mesenteric artery)

4. Low interaortacaval (between aorta and caval vein and below the lower mesenteric artery

5. High para-caval (right side of the caval vein and between right renal vein and above the level of the lower mesenteric artery)

6. Low para-caval (right side of the caval vein and below the level of the lower mesenteric artery)

7. Iliaca communis left

8. Illaca communis right

9. External iliaca left

10. External iliaca right 
11. Obturator left

12. Obturator right

The surgeon also has to register the location of the lymphatic tissue removed, related to the lymph node sampling following the removal of the sentinel node(s).

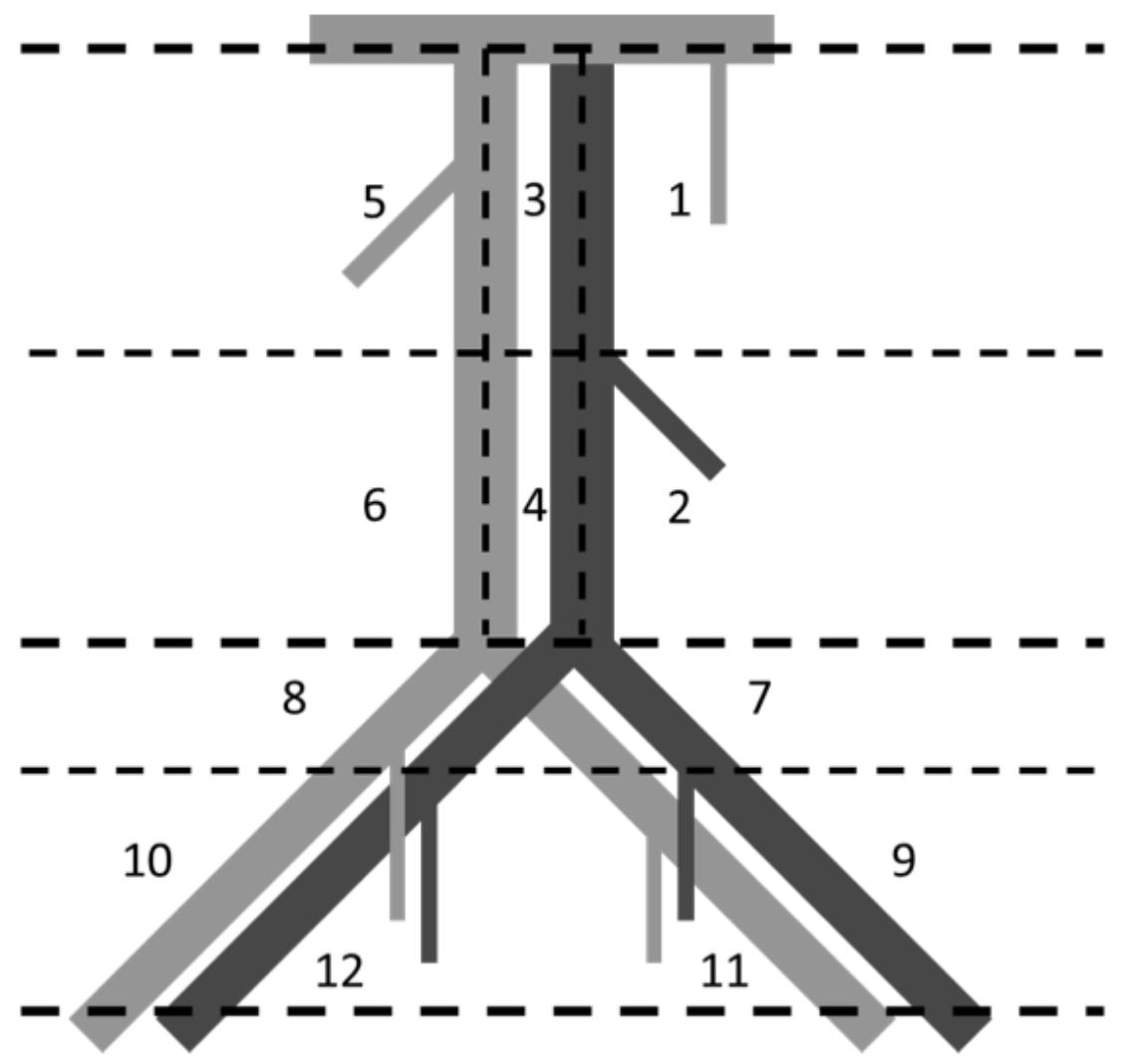




\section{Withdrawal of individual subjects and replacement:}

Subjects can leave the study at any time for any reason if they wish to do so without any consequences. The investigator can decide to withdraw a subject from the study for urgent medical reasons. Patients with a benign ovarian tumour on frozen section during surgery will also be excluded from the study. These patients will be replaced by a new study subject until 20 completed sentinel node procedures are performed.

\section{Discussion}

Recognition of sentinel nodes with blue dye and the gamma probe during surgery is less reliable than by making a scintigram. Therefore ideally, as in breast and vulvar cancer, a scintigram is made before surgery to recognize the sentinel nodes so that during surgery no sentinel nodes will be missed. However, this cannot be accomplished in patients with ovarian cancer, because the tracers are injected during surgery. However, we expect that missing sentinel nodes in patients with ovarian cancer occurs less frequent because the area where the sentinel nodes can occur can easily be visualised. Nevertheless, by making a scintigram 24 hours after surgery, at least an impression is obtained on the incidence of unrecognized sentinel lymph nodes.

\section{List of abbreviations}

EOC: epithelial ovarian cancer

FIGO: International Federation of Gynecology and Obstetrics

PET: Positron emission tomography

MRI: Magnetic resonance imaging

CT: computed tomography

TAH: total abdominal hysterectomy

BSO: bilateral salpingo-oophorectomy

MBq: megabecquerel 


\section{References}

1. Epitheliaalovariumcarcinoom. 2009 [cited 2010 19-02]; Available from: http://www.oncoline.nl/index.php?pagina=/richtlijn/item/pagina.php\&id $=28908 \&$ richtlijn_id=631.

2. Cancer Facts \& Figures 2009. Estimated New Cancer Cases and Deaths by Sex, US: American Cancer Society;2009.

3. Panici PB, Angioli R. Role of lymphadenectomy in ovarian cancer. Best Pract Res Clin Obstet Gynaecol 2002;16:529-551.

4. Tan DS, Agarwal R, Kaye SB. Mechanisms of transcoelomic metastasis in ovarian cancer. Lancet Oncol 2006;7:925-934.

5. Burghardt E, Girardi F, Lahousen M, Tamussino K, Stettner H. Patterns of pelvic and paraaortic lymph node involvement in ovarian cancer. Gynecol Oncol 1991;40:103-106.

6. Benedetti-Panici P, Greggi S, Maneschi F, Scambia G, Amoroso M, Rabitti C, Mancuso S. Anatomical and pathological study of retroperitoneal nodes in epithelial ovarian cancer. Gynecol Oncol 1993;51:150-154.

7. Onda T, Yoshikawa H, Yokota H, Yasugi T, Taketani Y. Assessment of metastases to aortic and pelvic lymph nodes in epithelial ovarian carcinoma. A proposal for essential sites for lymph node biopsy. Cancer 1996;78:803-808.

8. Tsumura N, Sakuragi N, Hareyama H, Satoh C, Oikawa M, Yamada H, Yamamoto R, Okuyama K, Fujino T, Sawaga T, Fujimoto S. Distribution pattern and risk factors of pelvic and para-aortic lymph node metastasis in epithelial ovarian carcinoma. Int J Cancer 1998;79:526-530.

9. Sakuragi N, Yamada H, Oikawa M, Okuyama K, Fujino T, akuragiagawa T, Fujimoto S. Prognostic significance of lymph node metastasis and clear cell histology in ovarian carcinoma limited to the pelvis (pT1M0 and pT2M0). Gynecol Oncol 2000;79:251-255.

10. Suzuki M, Ohwada M, Yamada T, Kohno T, Sekiguchi I, Sato I. Lymph node metastasis in stage I epithelial ovarian cancer. Gynecol Oncol 2000;79:305308.

11. Morice P, Joulie F, Camatte S, Atallah D, Rouzier R, Pautier P, Pomel C, Lhommé C, Duvillard P, Castaigne D. Lymph node involvement in epithelial 
ovarian cancer: analysis of 276 pelvic and paraaortic lymphadenectomies and surgical implications. J Am Coll Surg 2003;197:198-205.

12. Negishi H, Takeda M, Fujimoto T, Todo Y, Ebina Y, Watari H, Yamamoto R, Minakami H, Sakuragi N. Lymphatic mapping and sentinel node identification as related to the primary sites of lymph node metastasis in early stage ovarian cancer. Gynecol Oncol 2004;94:161-166.

13. Takeshima N, Hirai Y, Umayahara K, Fujiwara K, Takizawa K, Hasumi K. Lymph node metastasis in ovarian cancer: difference between serous and non-serous primary tumours. Gynecol Oncol 2005;99:427-431.

14. Maggioni A, Benedetti Panici P, Dell'Anna T, Landoni F, Lissoni A, Pellegrino A, Rossi RS, Chiari S, Campagnutta E, Greggi S, Angiolo R, Manci N, Calcagno M, Scambia G, Fossati R, Floriani I, Torri, Grassi R, Mangioni C. Randomised study of systematic lymphadenectomy in patients with epithelial ovarian cancer macroscopically confined to the pelvis. Br J Cancer 2006;18:699704.

15. Harter P, Gnauert K, Hils R, Lehmann TG, Fisseler-Eckhoff A, Traut A, du Bois A. Pattern and clinical predictors of lymph node metastases in epithelial ovarian cancer. Int J Gynecol Cancer 2007;17:1238-1244.

16. Nomura H, Tsuda H, Susumu N, Fujii T, Banno K, Kataoka F, Tominaga E, Suzuki A, Chiyoda T, Aoki D. Lymph node metastasis in grossly apparent stages I and II epithelial ovarian cancer. Int J Gynecol Cancer 2010;20:341345.

17. Ang D, Ng KY, Tan HK, Chung AY, Yew BS, Lee VK. Ovarian carcinoma presenting with isolated contralateral inguinal lymph node metastasis: a case report. Ann Acad Med Singapore 2007;36:427-430.

18. Oei AL, de Hullu JA, Grefte JM, Havenith BP. An enlarged groin node as first manifestation of a malignancy: don't forget the ovaries. Eur J Obstet Gynecol Reprod Biol 2008;138:240-242.

19. Fournier M, Stoeckle E, Guyon F, Brouste V, Thomas L, MacGrogan G, Floquet A. Lymph node involvement in epithelial ovarian cancer: sites and risk factors in a series of 355 patients. Int J Gynecol Cancer 2009;19:13071313. 
20. Carnino F, Fuda G, Ciccone G, Iskra L, Guercio E, Dadone D, Conte PF. Significance of lymph node sampling in epithelial carcinoma of the ovary. Gynecol Oncol 1997;65:467-472.

21. Kleppe M, Wang T, Van Gorp T, Slangen BFM, Kruse AJ, Kruitwagen RFPM. Lymph node metastasis in stages I and II ovarian cancer: a review. Gynecol Oncol 2011;123:610-614.

22. Benedet JL, Bender H, Jones H, 3rd, Ngan HY, Pecorelli S. FIGO staging classifications and clinical practice guidelines in the management of gynecologic cancers. FIGO Committee on Gynecologic Oncology. Int J Gynaecol Obstet 2000;70:209-262.

23. Carnino F, Fuda G, Ciccone G, Iskra L, Guercio E, Dadone D, Conte PF. Significance of lymph node sampling in epithelial carcinoma of the ovary. Gynecol Oncol 1997;65:467-72.2

24. Yuan Y, Tao XF, Lui SY. Computer tomography, magnetic resonance imaging, and positron emission tomography or positron emission tomography/computer tomography for detection of metastatic lymph nodes in patients with ovarian cancer: a meta-analysis. Eur J Radiol 2012;81:1002-1006.

25. Di Re F, Baiochocci G. Value of lymph node assement in ovarian cancer: status of the art at the end of the second millennium. Int J Gynecol Cancer 2000;10:435-442.

26. El-Ghobashy AE, Saidi SA. Sentinel node sampling in gynaecological cancers: techniques and clinical applications. Eur J Surg Onc 2009;35:675-685.

27. de Hullu JA, Hollema H, Piers DA, Verheijen RH, van Diest PJ, Mourits MJ, Aalders JG, van Der Zee AG. Sentinel lymph node procedure is highly accurate in squamous cell carcinoma of the vulva. J Clin Oncol 2000;18:2811-2816.

28. Nyberg RH, Korkola P, Tech L, Mäenpää. Ovarian sentinel node, is it feasible? Int J Gynecol Cancer 2011;21:567-572.

29. Hayashida T, Jinno H, Sakata M, Takahasi M, Onishi M, Seki H. Superiority of radioactive over blue dye for sentinel lymph node detection in breast cancer. Eur Surg Res 2010;44:111-116. 
30. Eiriksson LR, Covens A. Sentinel lymph node mapping in cervical cancer: the future? BJOG 2012;119:129-133.

31. Bézu C, Coutant C, Salengro A, Daraï E, Rouzier R, Uzan S. Anaphylactic response to blue dye during sentinel node biopsy. Surg Onc 2011;20:55-59.

32. Dewachter P, Mouton-Faicre C, Trechot P, Lleu JC, Mertes PM. Severe anaphylactic shock with methylene instillation. Anesth Analg 2005;101:149-150.

33. Rzymski P, Wozniak J, Opala T, Wilczak M, Sajdak S. Anaphylatic reaction to methylene blue dye after laparoscopic chromopertubation. Int J Gynaecol Obstet 2003;81:71-72.

34. deKanter AY, Arends PPAM, Eggermont AMM, Wiggers T. Radiation protection for the sentinel node procedure in breast caner. Eur J Surg Oncol 2003;29:396-399. 



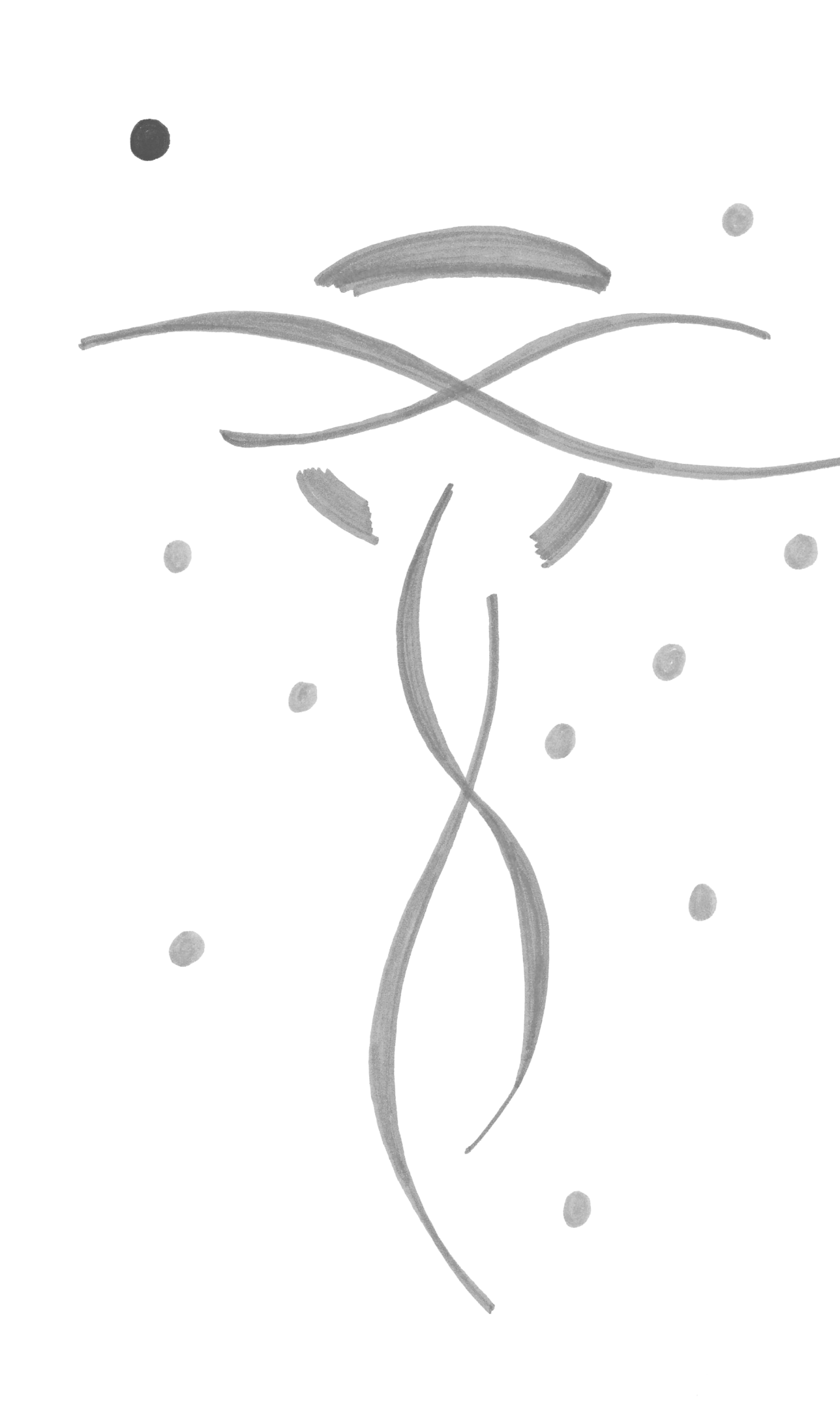




\section{Chapter 5}

\section{The detection of sentinel}

nodes in ovarian cancer: a feasibility study

Kleppe M, Brans B, Van Gorp T, Slangen BFM, Kruse AJ, Pooters INA, Lotz MG, Van de Vijver KK, Kruitwagen RFPM Journal of Nuclear Medicine 2014;55:1799-1804 


\section{Abstract}

Few sentinel node (SN) studies in ovarian cancer have been reported, mainly because of the risk of tumour dissemination associated with the injection of tracers into the ovarian cortex. To our knowledge, the injection of tracers into the ovarian ligaments has not been explored. The aim of this study was to determine the feasibility of the SN procedure in ovarian cancer with tracer injection into the ovarian ligaments and to establish if the procedure is safe for the health care workers.

\section{Methods}

The study included patients who were at high risk of ovarian malignancy. Blue dye and radioactive colloid were injected into the proper ovarian ligament and suspensory ligament of the ovary. To measure the professional radiation exposure, during three procedures ring dose meters were worn by the surgeon, the theatre nurse, and the pathologist.

\section{Results}

A SN procedure was performed in 21 patients and at least one SN-location was identified in all patients using the gamma probe before retroperitoneal exploration. SNs were located in the para-aortic and para-caval regions only in $67 \%$ of the patients, in the pelvic region only in $9 \%$, and in both the paraaortic/para-caval and pelvic regions in $24 \%$ of patients. All but two SNs were found on the ipsilateral side. In six patients who underwent retroperitoneal exploration, one to four SNs were identified using the gamma probe and resected. Blue-stained SNs were detected in two patients. Positive SNs were detected in one patient with lymph node metastases. The amount of radiation exposed to the surgeon, theatre nurse and pathologist did not exceed the safe limit.

\section{Conclusion}

The identification of SN(s) in all cases suggest that the SN procedure performed by injection of tracers in the ovarian ligaments is feasible and promising. The procedure is safe for the involved personnel. Further investigation is necessary to determine the clinical application of this new technique. 


\section{Introduction}

Epithelial ovarian cancer (EOC) is the leading cause of death among gynaecological malignancies, and the high mortality rate is partially attributed to the fact that up to $75 \%$ of patients are diagnosed with advanced stage EOC. In patients with clinical early-stage EOC, a staging procedure is recommended, which includes a total abdominal hysterectomy with bilateral salpingo-oöphorectomy, omentectomy, numerous peritoneal biopsies, and a pelvic and para-aortic lymph node dissection. Although the International Federation of Obstetrics and Gynaecology (FIGO) recommends a complete pelvic and para-aortic lymphadenectomy, the extent of lymph node dissection differs greatly from centre to centre..$^{1-3}$

In patients with FIGO stage I-IIa ovarian cancer, adjuvant chemotherapy could be omitted after a comprehensive staging procedure. Adjuvant chemotherapy is obligatory in patients with FIGO stage IIb-IV ovarian cancer. The omission of an adequate lymphadenectomy in clinical early-stage disease results in the underdiagnosis of stage III disease in approximately in $10-20 \%$ of patients, leading to the exclusion of adjuvant chemotherapy with curative intent.4,5

Lymphatic metastases of EOC mainly occur in the para-aortic and para-caval lymph nodes, although they can also be found in the pelvic lymph nodes. ${ }^{5-10}$ Tumour cells travel along the lymph vessels that accompany the ovarian artery and vein in the suspensory ligament up to the high para-aortic and para-caval regions. In pelvic lymph node metastasis, tumour cells are likely to move along lymph vessels in the proper ovarian ligament to the para-uterine vessels in the broad ligament, and further towards the iliac vessels. Therefore, lymph nodes need to be removed from different anatomical regions (the para-aortic and para-caval regions, the common, internal and external iliac vessels, and the obturator fossa). The detection of metastases is positively correlated with the number of lymph nodes removed. ${ }^{11,12}$ Complete pelvic and para-aortic lymphadenectomy was shown to result in the detection up to 250 or more lymph nodes. ${ }^{9}$ An overall incidence of lymph node metastasis of approximately 14\% was reported in clinical early-stage EOC, with a higher incidence in grade III tumours (20\%) and in the serous histological subtype $(23 \%)$ than in grade I (4\%) and mucinous tumours (3\%). ${ }^{5}$ Because positive lymph nodes can be missed during lymph node sampling, systematic lymphadenectomy is 
considered the gold standard. However, this is a radical procedure that is associated with obvious morbidity, including nerve and vessel injury, increased blood loss, increased operating time, and the formation of lymphocysts and lymphedema. ${ }^{10,12,13}$

The sentinel node (SN) procedure enables the identification of the first lymph node receiving primary lymphatic flow (the so-called SN). Histopathology results indicate the nodal status of all lymph nodes in a certain anatomical region. The detection of a negative SN suggests that the remaining lymph nodes are not involved. As a consequence, the patient may be spared a radical lymphadenectomy and thus the associated morbidity. The SN technique has been proven effective in vulvar and breast cancer, and SN studies in cervical and uterine cancers are currently under way. ${ }^{14-17}$ For the ovary, SN studies are scarce, partly because of the risk of tumour dissemination associated with the injection of tracers into the ovarian cortex. ${ }^{16-19}$

In the current study, we examined the effect of tracer injection into the ovarian ligaments instead of the ovarian cortex during the SN procedure.

The objective of this technique was to prevent spillage of cyst fluid and malignant cells and to inject the tracer in the proximity of the draining lymph vessels in the ovarian ligaments. Moreover, this technique enables the repeated injection of the tracer in the same location, irrespective of the size of the ovarian mass. To the best of our knowledge, the injection of tracers into the ovarian ligaments has not been explored previously. The aim of the present study was to determine the feasibility of the SN procedure performed by the injection of tracers into the ovarian ligaments and to establish if the procedure is safe for the health care workers.

\section{Materials and methods}

\section{Patients}

Patients diagnosed with a pelvic mass suspicious for a malignant ovarian tumour who were treated in Maastricht University Medical Centre were eligible for inclusion in the study ${ }^{20}$. All patients provided fully informed consent prior to enrolment in the study, and the protocol was approved by the Local Ethics 
Committee (approval number: NL40323.068.12) (clinical trial registration number is NCT01734746). Exclusion criteria were as follows: previous surgery on one or both ovaries; previous vascular surgery of the aorta, caval vein, and/or iliac vessels; previous lymphadenectomy or lymph node sampling in the iliac or paraaortic region; a history of malignant lymphoma; a history of a malignant tumour in the abdominal cavity; previous allergic reaction to blue dye or human albumin; and pregnant or lactating patients.

\section{SN procedure}

After opening the abdomen and before the removal of the enlarged and suspicious ovary, blue dye and the radioactive isotope were injected on the dorsal and ventral side of the proper ovarian ligament and the suspensory ligament, close to the ovary and just underneath the peritoneum (Figure 1).

Figure 1. Location of injection of the tracers.

Black star: suspensory ligament of the ovary. Gray star: proper ovarian ligament. Tracers were injected on the ventral and dorsal side of both ligaments.

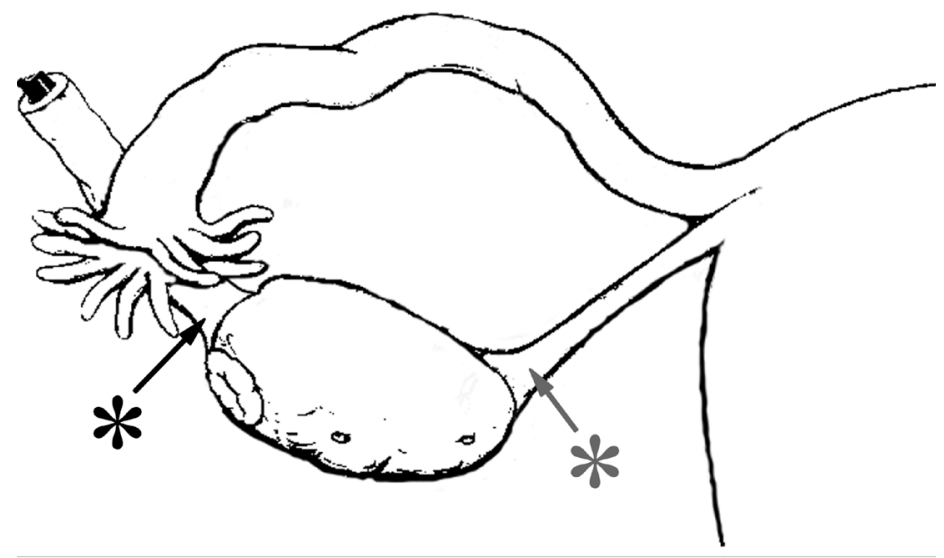

Each of the four injections contained 0.2-0.5 mL blue dye (Blue Patente V, 25 $\mathrm{mg} / \mathrm{ml}$, Guerbet Nederland B.V, Gorinchem, The Netherlands) and 20-MBq technetium-99-m-labeled albumin nanocolloid with a particle size $<80 \mathrm{~nm}(99 \mathrm{~m}$ 
Tc-nanocolloid or Nanocoll, GE Healthcare, Eindhoven, The Netherlands). After a 15-minute time interval, the adnexal mass was removed and sent to the pathologist for the examination of frozen sections.

This was followed by examination of the different lymph node regions for the presence of SNs using a standard $12 \mathrm{~mm}$ straight gamma probe (Node Seeker $囚$, Intra Medical Imaging, Santa Monica, CA, USA). The location was considered positive if the counts were at least 10 -fold higher than the background radiation. If a benign or borderline ovarian tumour was detected, lymph node dissection was not performed. Nevertheless, in these patients potential SNs were once more localised transperitoneally using the gamma probe. Six different regions were used to describe the localization of the hot spots with the gamma probe, as follows: upper and lower para-aortic regions separated by the origin of the inferior mesenteric artery, upper and lower para-caval regions, and right and left pelvic regions.

On identification of a malignant mass, the retroperitoneal space was opened and the SN(s) was localized with the gamma probe and/or visually (blue dye). The surgeon recorded the number and location of the resected SN(s), with 12 different regions used to describe their localization, as follows: upper and lower para-aortic regions, upper and lower interaortocaval regions, upper and lower para-caval regions, right and left common iliac regions, right and left external iliac regions, and right and left obturator fossa regions. After removal of the SNs, the location from which they were removed was re-examined for the presence of radioactivity. If the radioactivity was $<10 \%$ of that in the initial positive lymph node, no further action was taken. After removal of the SN(s), a complete standard staging procedure was performed including a comprehensive sampling of other lymph nodes in the different anatomical locations. The surgeon also recorded the location of the removed non-SNs.

\section{Histopathology}

SN(s) and non-SN(s) were examined separately. Non-SNs were cut into single sections and stained with hematoxylin and eosin (H\&E), according to the standard protocol for lymph node examination. SNs were cut into $2 \mathrm{~mm}$ sections for $\mathrm{H} \& \mathrm{E}$ staining. H\&E-negative SNs in the first section were further cut into $500 \mu \mathrm{m}$ 
sections and analyzed for the presence of micrometastases $(<2 \mathrm{~mm})$. At each step, immunohistochemical staining with cytokeratin MNF-116 was performed. Metastases $>2 \mathrm{~mm}$, those between 0.2 and $2 \mathrm{~mm}$, and those $<0.2 \mathrm{~mm}$ were considered as macrometastases, micrometastases and isolated tumour cells, respectively.

\section{Sample size calculation}

A sample size of 20 evaluable patients was considered large enough to determine whether identification of $\mathrm{SN}(\mathrm{s})$ is feasible by injection of tracers into the ovarian ligaments.

\section{Measurement radiation exposure personnel}

To have an indication of the professional radiation exposure, during three procedures the radiation dose to which the surgeon, the theatre nurse, and the pathologist were exposed was measured. A ring dose meter, pointed towards the inside of the hand, was worn by these persons at the base of the index finger at the right and left hand during surgery and/or handling of tissues. Additionally, electronic dose meters were placed on the body at chest level.

\section{Results}

\section{Patients' characteristics}

A total of 22 patients were included in the study. The SN procedure was successfully completed in 21 of 22 patients (96\%). In one patient with a dermoid cyst, the high number of adhesions prevented access to the ovarian ligaments without interfering with the normal anatomy during injection of the tracer. Allergic or adverse reactions did not occur in any of the patients. The SN procedure was performed 7 times on the left side and 14 times on the right side. The final pathology results showed that seven patients had a benign tumour, eight had a borderline tumour, and six had malignant EOC. All frozen sections were correctly diagnosed and confirmed in the final pathology results. One patient was diagnosed with a primary ovarian tumour and endometrial cancer (grade I endometrioid type tumour with minimal myometrial invasion). Based on the staging procedure, intraabdominal tumour metastases were not identified in any of these patients. 


\section{Transperitoneal SN detection with the gamma probe after injection of the}

\section{tracer}

In all patients, at least one hot spot could be identified with the gamma probe within 15 minutes after injection of the radioactive tracer. Hot spots were found only in the para-aortic and para-caval regions in $67 \%$ of the patients, in the pelvic region only in $9 \%$, and in both the para-aortic/para-caval and pelvic regions in $24 \%$ of the patients (Table 1 ).

Table 1. Transperitoneal sentinel node detection with gamma probe.

\begin{tabular}{|c|c|c|c|c|c|}
\hline \multicolumn{3}{|c|}{ Patients } & \multicolumn{3}{|c|}{ Hot spot locations } \\
\hline Tumour location & Histology & Number & Only para- & Only pelvic & Both para- \\
\hline & & & /paracaval & & $\begin{array}{l}\text { paracaval } \\
\text { and pelvic }\end{array}$ \\
\hline \multirow[t]{3}{*}{ Ovary } & Benign & 7 & 6 & 0 & 2 \\
\hline & Borderline & 8 & 4 & 2 & 2 \\
\hline & Malignant & 5 & 3 & 0 & 1 \\
\hline \multirow{2}{*}{$\begin{array}{c}\text { Ovary and } \\
\text { endometrium }\end{array}$} & Both & 1 & 1 & 0 & 0 \\
\hline & malignant & & & & \\
\hline Total & & 21 & $14(67 \%)$ & $2(9 \%)$ & $5(24 \%)$ \\
\hline
\end{tabular}

Eight patients had more than one hot spot (two hot spots in seven and four hotspots in one patient). All but two of the $31 \mathrm{SN}$ locations were on the ipsilateral side (Figure 2a). Nearly all para-aortic SNs related to the left ovary were situated just below the renal vein and above the level of the inferior mesenteric artery, whereas the para-caval SNs related to the right ovary were lower, being mainly located at the level of the inferior mesenteric artery. 
Figure 2. Detection of sentinel nodes.

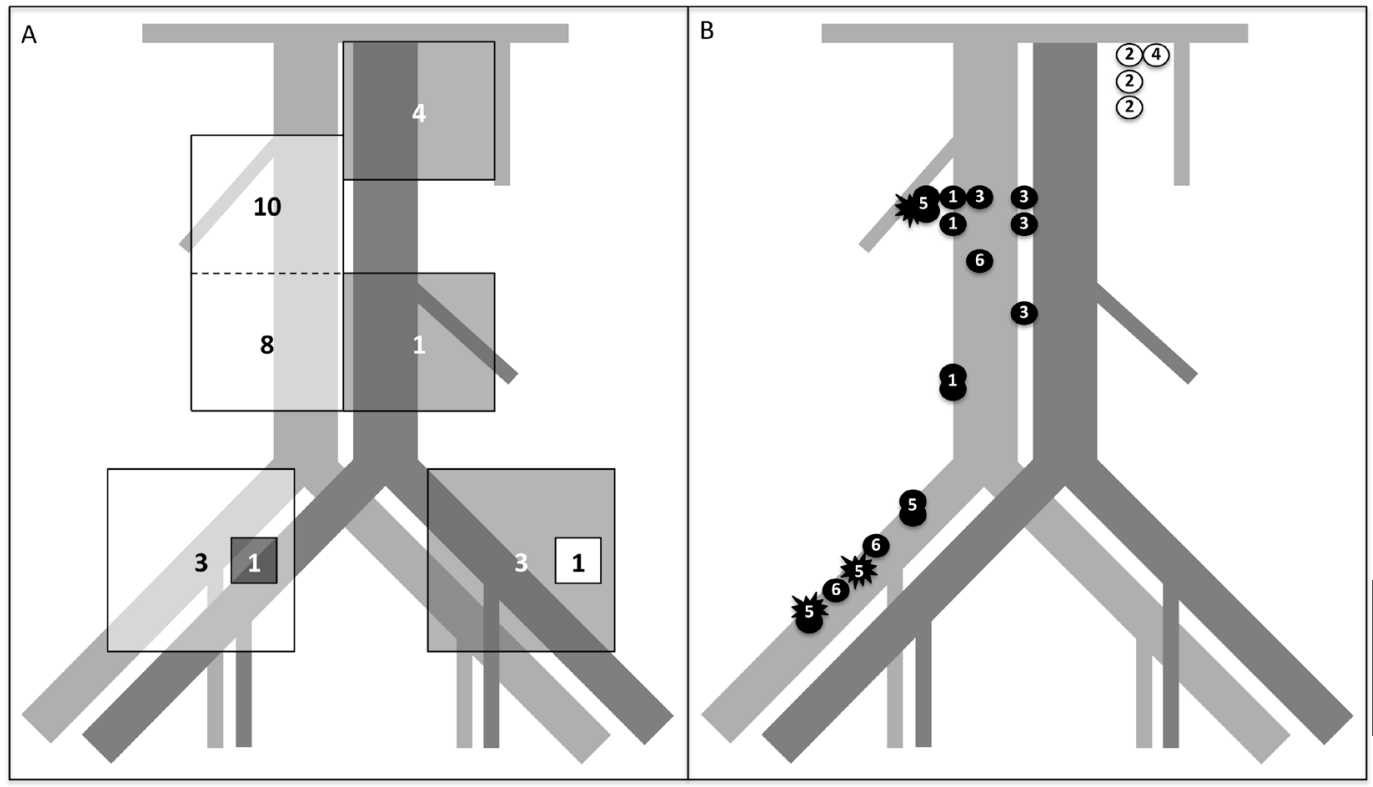

\section{a. Transperitoneal detection of hot spots}

Figure shows the location and number of hot spots found with the gammaprobe. White squares: location of the hotspots found in patients ( $\mathrm{n}=14$ ) with a tumour on the right side.

Grey squares: location of the hotspots found in patients $(n=7)$ with a tumour on left side.

\section{b. Location of SNs at retroperitoneal exploration}

Numbers correspond with patients' number as described in table 2 .

Black circles: location of the sentinel nodes found in patients with tumour on the right side. Black stars: location of the sentinel nodes containing metastasis.

\section{Retroperitoneal SN detection (Table 2, Figure 2b)}

Retroperitoneal exploration with lymph node dissection was performed in six patients. In four of these patients, additional SNs were detected with the gamma probe at the location of the hot spot during retroperitoneal exploration. Furthermore, after opening the peritoneum, additional SNs were identified in 
other regions in three patients. In all six patients, the SNs were clearly identified with the radioactive tracer using the gamma probe. In two patients (patient number one and number five), the SNs also showed blue staining. All para-aortic SNs related to the left ovary were situated just below the renal vein. The para-caval SNs related to the right ovary were mainly located at the level of the inferior mesenteric artery.

Table 2. Overview of patients with lymph nodes removed.

\begin{tabular}{|c|c|c|c|c|c|c|}
\hline \multirow[t]{3}{*}{ Patient } & & \multicolumn{5}{|c|}{ Retroperitoneal detection } \\
\hline & $\begin{array}{c}\text { Trans } \\
\text { peritoneal } \\
\text { detection }\end{array}$ & & tinel nodes & & Non-sentinel & \\
\hline & locations & Locations & Number ${ }^{\dagger}$ & Metastases & Number & Metastases \\
\hline 1 & 1 & 3 & 4 & 0 & 11 & 0 \\
\hline 2 & 1 & 3 & 3 & 0 & 7 & 0 \\
\hline 3 & 1 & 4 & 4 & 0 & 8 & 0 \\
\hline 4 & 1 & 1 & 1 & 0 & 20 & 0 \\
\hline $5^{*}$ & 2 & 4 & 8 & 3 & 8 & 1 \\
\hline 6 & 2 & 3 & 3 & 0 & 11 & 0 \\
\hline
\end{tabular}

* Patient with primary ovarian and endometrial cancer.

† Number of lymph nodes recognized by histology.

\section{Histopathology}

A total of 18 tissue specimens were positive for SNs. In the final histological examination, more than one lymph node was identified in four of these tissue specimens (Table 2, Figure 2b).

In one patient (patient number five) metastases were found in the resected SNs.

Three out of four SNs removed contained micrometastases, which were also detected in one of the remaining eight lymph nodes removed. 


\section{Radiation exposure personnel}

Of three procedures performed, only the dose meters at the extremities of the surgeon gave values above background radiation level. The total dose measured during three procedures was $0.06 \mathrm{mSv}$ at the dominant hand of the surgeon and $0.17 \mathrm{mSv}$ at the non-dominant hand.

\section{Discussion}

In the present study, we showed that perioperative injection of tracers into the ovarian ligaments is a feasible method for the detection of SNs. One or more SNs were detected with the gamma probe in all the patients $(n=21)$ who underwent the SN procedure. Hot spots were found isolated in the para-aortic and para-caval regions in most patients (67\%), in the pelvic region only in $9 \%$, and in both the para-aortic/para-caval and pelvic regions in $24 \%$ of the patients. In all but two patients, the SNs were ipsilateral to the injection site. These findings correlate well with the results of a review on the incidence and location of lymph node metastases in clinical stage I-II EOC, in which metastases were found isolated in the para-aortic region in 50\%, in the pelvic region in $20 \%$, and in both the paraaortic and pelvic region in the remaining $30 \%$ of patients. ${ }^{5}$

SN studies in ovarian cancer are scarce. Negishi et al. used injection of an activated charcoal solution into the ovarian capsule to identify SNs and reported the detection of SNs in the para-aortic region in all 11 patients analysed (overall eighteen SNs) and in the pelvic region in four patients (overall four SNs). ${ }^{18}$ Nyberg et al. analysed 16 patients with high-risk uterine cancer by injecting technetium and blue dye into the right or left ovary and detected one to three SNs in 15 of the 16 patients. ${ }^{19}$ The SNs $(n=30)$ were all located in the para-aortic region. The SNs related to the left ovary were mainly (64\%) located above the level of the inferior mesenteric artery, whereas most SNs of the right ovary (94\%) were found below the level of the inferior mesenteric artery, which is in agreement with our findings. In both studies, the tracer was injected into the ovary. However, the injection of tracers into the ovary can be difficult when bulky ovarian masses are present. Furthermore, the injection of tracers into the ovarian capsule is associated with a potential risk of tumour dissemination. ${ }^{16,17}$ This probably explains why, hitherto, 
SN detection in ovarian cancer has not been adopted in clinical practice. The adnexal mass should preferably be removed intact, without rupture. The spillage of malignant cells within the peritoneal cavity will increase the patient's stage. Although the effect on prognosis of peroperative spill still is controversial, current practice by the FIGO guidelines is to avoid spillage. ${ }^{21}$ In the present study, tracers were injected in the ovarian ligaments to overcome the risk of tumour dissemination. In only one out of the 22 patients, we were unable to access the ovarian ligaments due to adhesions potentially caused by leakage of a dermoid cyst and not the size of the tumour.

The exact timing for the detection of positive lymph nodes remains undefined. The time period should be long enough to allow the tracers to be transported to the SNs, but an increased lag time requires prolonged general anaesthesia and increased costs. Therefore, delaying surgery for a period longer than 15 minutes was deemed not to be feasible in daily practice. Negishi et al. and Nyberg et al. waited for 10 minutes and 10-21 minutes after injection, respectively. ${ }^{18} 19$ Intraoperative SN procedures in case of cervical and endometrial cancer also use comparable time-intervals. ${ }^{16}$ In the present study, we used a 15-minute interval between injection of the tracers and resection of the adnexal mass, and at least one SN was identified in all patients within this period. In case of an ovarian malignancy, the time interval between injection of the tracers and detection and resection of the $\mathrm{SN}(\mathrm{s})$ was at least 50 minutes (including 15 minutes waiting time after injection, and time spent for removal of the ovarian tumour and frozen section analysis). As described in the results, in four patients additional SNs within the same region were discovered after opening of the retroperitoneum and in three patients additional SNs were discovered in other regions. Identifying more lymph nodes during retroperitoneal exploration could be explained by a better and more precise accessibility of the lymph node locations but possibly also because of a longer time interval after injection of the tracer. Indeed, additional work needs to be done to optimize the SN detection technique in ovarian cancer. Items to be determined include the optimal colloid particle sizes which results in the fastest migration and best retention in SNs. In a study by Glass et al., examining the kinetics of three different radioactive agents in cutaneous melanoma, detection at 
30 min. was more appropriate than late imaging. ${ }^{22}$ Paganelli et al. studying breast cancer, showed that the number of SN's detected strongly related to the size of the particle, i.e. the smaller the particles, the greater the number of lymph nodes. ${ }^{23}$ However, all these data may be organ dependent, so it is presently unknown if this is also the case in ovarian cancer. Moreover, newly developed hybrid SN detection agents, combining radioactivity with fluorescence and/or paramagnetic capabilities, alternative operative techniques such as multiphased injection, and new intraoperative imaging technologies may provide additional advantages. ${ }^{24-27}$

In general, lymphatic mapping involves the injection of a blue dye or a radioactive isotope. SN studies in different primary tumours showed that the detection rate is highest when a radioactive isotope and the blue dye are used in combination. ${ }^{28,29}$ In the present study, blue-stained SNs were identified in only two out of six patients during retroperitoneal exploration. The long interval between the injection of the tracer and retroperitoneal exploration of at least 50 minutes may have caused the blue dye to fade before the surgical procedure. A SN study in cervical cancer reported the detection of blue-stained lymph nodes after a median time of 7 minutes after injection, with the lymph nodes remaining blue for a median time of 21 minutes. $^{30}$ In future studies the utility of the blue dye as a tracer in case of ovarian cancer should be examined in more detail.

A pelvic and para-aortic lymphadenectomy or, at least, comprehensive lymph node sampling in both the pelvic and para-aortic/para-caval regions is an essential part of staging in clinical early-stage ovarian cancer. The presence of lymph node metastases is an indication for adjuvant chemotherapy. The main purpose of the SN technique in clinical early-stage ovarian cancer is the reduction of treatmentrelated morbidity in patients without metastatic lymph nodes while avoiding the risk of missing involved lymph nodes. Approximately $14 \%$ of patients with clinical early-stage disease have lymph node metastases. ${ }^{5}$ As a consequence, $86 \%$ of patients are not likely to benefit from a lymphadenectomy, although they are at risk for surgery-related morbidity. Complete pelvic and/or para-aortal lymphadenectomies are associated with increased peri- and post-operative morbidities, which mainly include lymphocysts and lymphorrhea. ${ }^{10,12,13}$ 
Furthermore, the use of SN procedure as a substitute for a lymph node sampling may even be associated with a higher sensitivity for the identification of involved lymph nodes.

The SN procedure is already proven to be accurate and cost-effective, related to a marked improvement in post-operative morbidity, in different types of cancer such as breast and vulvar cancer. ${ }^{31-33}$ However, in patients with a complex adnexal mass, the SN procedure should be performed before the diagnosis EOC can be made based on frozen section. As a consequence a certain number of patients obtain the SN procedure while having a benign adnexal mass. Therefore the added costs of SN procedures in patients with a benign ovarian tumour should also be taken into account. For these patients the SN procedure does not give any advances, however the risk of complications and side effects of the procedure is low. ${ }^{34-36}$

The radiation exposure measurements on surgeon, theatre nurse and pathologist during three procedures only showed values above background radiation level for the surgeon. The maximum value was measured at the non-dominant hand being $<0.1 \mathrm{mSv}$ per procedure. The difference between the dominant hand and the nondominant hand is in accordance with a previous study of de Kanter et al. who examined radiation exposure of personnel during SN procedure in breast cancer. ${ }^{37}$ The difference between the dominant hand and the non-dominant hand is caused by the operating technique. The non-dominant hand presents the tissue to be removed by the dominant hand, resulting in a shorter distance between nondominant hand and radioactive tissue. The maximum hand dose for non-exposed and exposed workers is 50 and $500 \mathrm{mSv} /$ year respectively. This means that no dose limits are exceeded and there is no additional risk for the involved personnel, if the number of procedures remains below 500 procedures/person/year.

The present study has limitations. First, a limited number of patients were included in the analysis mostly because ovarian cancers are often diagnosed an advanced stage of the disease. And although SNs were detected in all 21 patients who received the injection of tracers, the high number of benign and borderline 
ovarian tumours resulted in the resection of lymph nodes in only a small group of patients. In planning the study design, we presumed that in approximately $60 \%$ of the patients with a clinical suspicion of ovarian tumour, frozen section during surgery would be confirmatory. This calculation was based on the accuracy of the Risk of Malignancy Index (sensitivity 78\% and specificity 87\%) and a prevalence of malignancies within our study population of $20 \%{ }^{38}$ However, eight out of 14 malignancies were defined as borderline tumours in which case no lymph nodes are resected as part of the staging procedure. The numbers of cases in this study are too small to reach conclusions regarding false negative SNs based on histology. Second, the one patient with lymph node metastasis had an ovarian as well as an endometrial tumour. The positive lymph nodes could be related to the synchronous endometrial tumour. Further investigation including a larger number of patients in a multicentre study is necessary to determine the clinical application of this innovative technique.

\section{Conclusion}

In the present study we showed that the SN procedure performed through the injection of tracers into the ovarian ligaments is feasible and promising. The procedure is safe for the involved personnel. Injection of radioactive tracers resulted in the identification of SNs in all patients, suggesting that the technique could potentially be incorporated into routine clinical practice in patients with early-stage ovarian cancer. The SN procedure could be a cost-effective method to reduce the morbidity and rate of complications associated with complete pelvic and para-aortic lymphadenectomy. Plans for a multicentre prospective trial are currently underway to explore the benefits of the SN technique based on the injection of tracers in the ovarian ligaments in patients with clinical early-stage ovarian cancer. 


\section{References}

1. Benedet JL, Bender H, Jones H, $3^{\text {rd }}$, Ngan HY, Pecorelli S. FIGO staging classifications and clinical practice guidelines in the management of gynecologic cancers. FIGO Committee on Gynecologic Oncology. Int J Gynaecol Obstet 2000;70:209-262.

2. Angioli R, Plotti F, Palaia I, Calcagno M, Montera R, Cafà EV, Sereni MI, Panici PB. Update on lymphadenectomy in early and advanced ovarian cancer. Curr Opin Obstet Gynecol 2008;20:34-39.

3. Di Re F, Baiocchi G. Value of lymph node assessment in ovarian cancer: status of the art at the end of the second millennium. Int J Gynecol Cancer 2000;10:435-442.

4. Piver MS, Barlow JJ, Shashikant BL. Incidence of subclinical metastases in stage I and II ovarian carcinoma. Obstet Gynecol 1978;52:100-104.

5. Kleppe M, Wang T, Van Gorp T, Slangen BFM, Kruse AJ, Kruitwagen RFPM. Lymph node metastasis in stages I and II ovarian cancer: a review. Gynecol Oncol 2011;123:610-614.

6. Onda T, Yoshikawa H, Yokota H, Yasugi T, Taketani Y. Assessment of metastases to aortic and pelvic lymph nodes in epithelial ovarian carcinoma. A proposal for essential sites for lymph node biopsy. Cancer 1996;78:803-808.

7. Tsumura N, Sakuragi N, Hareyama H, Satoh C, Oikawa M, Yamada H, Yamamoto R, Okuyama K, Fujino T, Sawaga T, Fujimoto S. Distribution pattern and risk factors of pelvic and para-aortic lymph node metastasis in epithelial ovarian carcinoma. Int J Cancer 1998;79:526-530.

8. Morice P, Joulie F, Camatte S, Atallah D, Rouzier R, Pautier P, Pomel C, Lhommé C, Duvillard P, Castaigne D. Lymph node involvement in epithelial ovarian cancer: analysis of 276 pelvic and paraaortic lymphadenectomies and surgical implications. J Am Coll Surg 2003;197:198-205.

9. Harter P, Gnauert K, Hils R, Lehmann TG, Fisseler-Eckhoff A, Traut A, du Bois A. Pattern and clinical predictors of lymph node metastases in epithelial ovarian cancer. In J Gynecol Cancer 2007;17:1238-1244.

10. Ditto A, Martinelli F, Reato C, Kusamura S, Solima E, Fontanelli R, Haeusler E, Raspagliesi F. Systematic para-aortic and pelvic lymphadenectomy in 
early stage epithelial ovarian cancer: a prospective study. Ann Surg Oncol 2012;19:3849-3855.

11. Carnino F, Fuda G, Ciccone G, Iskra L, Guercio E, Dadone D, Conte PF. Significance of lymph node sampling in epithelial carcinoma of the ovary. Gynecol Oncol 1997;65:467-472.

12. Maggioni A, Benedetti Panici P, Dell'Anna T, Landoni F, Lissoni A, Pellegrino A, Rossi RS, Chiari S, Campagnutta E, Greggi S, Angiolo R, Manci N, Calcagno M, Scambia G, Fossati R, Floriani I, Torri, Grassi R, Mangioni C. Randomised study of systematic lymphadenectomy in patients with epithelial ovarian cancer macroscopically confined to the pelvis. British J of Cancer 2006;95:699-704.

13. Van de Poll-Franse L, Pijnenborg JMA, Boll D, Vos MC, van den Berg H, Lybeert ML, de Winter K, Kruitwagen RF. Health related quality of life and symptoms after pelvic lymphadectomy or radiotherapy vs. no adjuvant regional treatment in early-stage endometrial carcinoma: a large population-based study. Gynecol Oncol 2012;127:153-160.

14. de Hullu JA, Hollema H, Piers DA, Verheijen RH, van Diest PJ, Mourits MJ, Aalders JG, van Der Zee AG. Sentinel lymph node procedure is highly accurate in squamous cell carcinoma of the vulva. J Clin Oncol 2000;18:2811-2816.

15. Mansel RE, Fallowfield L, Kissin M, Goyal A, Newcombe RG, Dixon JM, Yiangou C, Horgan K, Bundred N, Monypenny I, England D, Sibbering M, Abdullah TI, Barr L, Chetty U, Sinnett DH, Fleissig A, Clarke D, Ell PJ. Randomized multicenter trial of sentinel node biopsy versus standard axillary treatment in operable breast cancer: the ALMANAC Trial. J Natl Cancer Inst 2006;98:599-609.

16. El-Ghobashy AE, Saidi SA. Sentinel node sampling in gynaecological cancers: techniques and clinical applications. Eur J Surg Onc 2009;35:675-685.

17. Giammarile F, Vidal-Sicart S, Valdés Olmos RA. Uncommon applications of sentinel lymph node mapping: urogenital cancers. Q J Nucl Med Mol Imaging 2014;58:161-179.

18. Negishi H, Takeda M, Fujimoto T, Todo Y, Ebina Y, Watari H, Yamamoto R, Minakami H, Sakuragi N. Lymphatic mapping and sentinel node 
identification as related to the primary sites of lymph node metastasis in early stage ovarian cancer. Gynecol Oncol 2004;94:161-166.

19. Nyberg RH, Korkola P, Tech L, Mäenpää J. Ovarian sentinel node, is it feasible? Int J Gynecol Cancer 2011;21:567-572.

20. Kleppe M, Van Gorp T, Slangen BFM, Kruse AJ, Brans B, Pooters IN, Van de Vijver KK, Kruitwagen RFPM. Sentinel node in ovarian cancer: study protocol for a phase 1 study. Trials 2013;14:47.

21. Prat J. Staging classification for cancer of the ovary, fallopian tube, and peritoneum. Int J Gynecol Obstet 2014;124:1-5.

22. Glass EC, Essner R, Morton DL. Kinetics of three lymphoscintigraphic agents in patients with cutaneous melanoma. J Nucl Med 1998;39:1185-1190.

23. Paganelli G, De Cicco C, Cremonesi M, Prisco G, Calza P, Luini A, Zucali P, Veronesi U. Optimized sentinel node scintigraphy in breast cancer. Q J Nucl Med 1998;42:49-53.

24. Ahmed M, Purushotham AD, Douek M. Novel techniques for sentinel lymph node biopsy in breast cancer: a systematic review. Lancet Oncol 2014;15:351-362.

25. Tsuchimochi M, Hayama K. Intraoperative gamma cameras for radioguided surgery: technical characteristics, performance parameters, and clinical applications. Phys Med 2013;29:126-138.

26. Schaafsma BE, van der Vorst JR, Gaarenstroom KN, Peters AA, Verbeek FP, de Kroon CD, Trimbos JB, van Poelgeest MI, Frangioni JV, van de Velde CJ, Vahrmeijer AL. Randomized comparison of near-infrared fluorescence lymphatic tracers for sentinel lymph node mapping of cervical cancer. Gynecol Oncol 2012;127:126-130.

27. Schaafsma BE, Verbeek FP, Peters AA, van der Vorst JR, de Kroon CD, van Poelgeest MI, Trimbos JB, van de Velde CJ, Frangioni JV, Vahrmeijer AL, Gaarenstroom KN. Near-infrared fluorescence sentinel lymph node biopsy in vulvar cancer: a randomised comparison of lymphatic tracers. BJOG 2013;120:758-764.

28. Hayashida T, Jinno H, Sakata M, Takahashi M, Onishi T, Seki H, Sato T, Nakahara T, Shigematsu N, Mukai M, Hibi T, Kitajima M, Kitagawa Y. 
Superiority of radioactive over blue dye for sentinel lymph node detection in breast cancer. Eur Surg Res 2010;44:111-116.

29. Eiriksson LR, Covens A. Sentinel lymph node mapping in cervical cancer: the future? BJOG 2012;119:129-133.

30. Levenback C, Coleman RL, Burke TW, Lin WM, Erdman W, Deavers M, Delpassand ES. Lymphatic mapping and sentinel node identification in patients with cervix cancer undergoing radical hysterectomy and pelvic lymphadenectomy. J Clin Oncol 2002;20:688-693.

31. Van der Zee AGJ, Oonk MH, De Hullu JA, Ansink AC, Vergote I, Verheijen RH, Maggioni A, Gaarenstroom KN, Baldwin PJ, Van Dorst EB, Van der Velden J, Hermans RH, van der Putten H, Drouin P, Schneider A, Sluiter WJ. Sentinel node dissection is safe in the treatment of early-stage vulvar cancer. J Clin Oncol 2008;26:884-889.

32. Verry H, Lord SJ, Martin A, Gill G, Lee CK, Howard K, Wetzig N, Simes J. Effectiveness and cost-effectiveness of sentinel lymph node biopsy compared with axillary node dissection in patients with early-stage breast cancer: a decision model analysis. British J of Cancer 2012;106:1045-1052.

33. Schulze T, Mucke J, Markwardt J, Schlag PM, Bembenek A. Long-term morbidity of patients with early breast cancer after sentinel lymph node biopsy compared to axillary lymph node dissection. J of Surg Oncol 2006;93:109-119.

34. Bézu C, Coutant C, Salengro A, Daraï E, Rouzier R, Uzan S. Anaphylactic response to blue dye during sentinel node biopsy. Surg Onc 2011;20:55-59.

35. Dewachter P, Mouton-Faicre C, Trechot P, Lleu JC, Mertes PM. Severe anaphylactic shock with methylene instillation. Anesth Analg 2005;101:149-150.

36. Rzymski P, Wozniak J, Opala T, Wilczak M, Sajdak S. Anaphylatic reaction to methylene blue dye after laparoscopic chromopertubation. Int J Gynaecol Obstet 2003;81:71-72.

37. de Kanter AY, Arends PPAM, Eggermont AMM, Wiggers T. Radiation protection for the sentinel node procedure in breast caner. Eur J Surg Oncol 2003;29:396-399. 
38. Geomini P, Kruitwagen RFPM, Bremer GL, Cnossen J, Mol BW. The accuracy of risk scores in predicting ovarian malignancy: a systematic review. Obstet Gynecol 2009;113:384-394. 



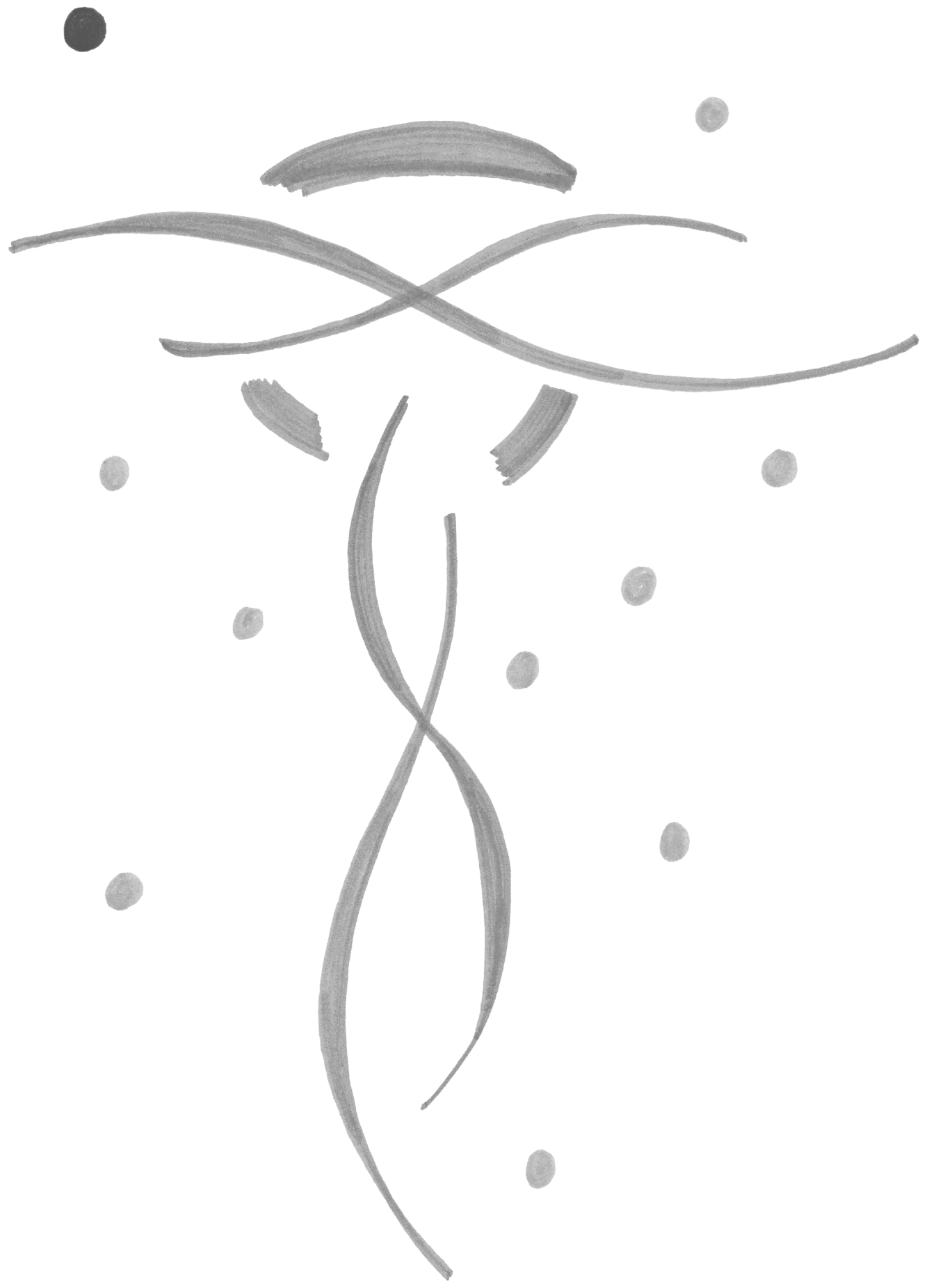




\section{Chapter 6}

\section{Understanding lymphatic}

drainage pathways of the

\section{ovaries to predict sites for}

\section{sentinel nodes in ovarian}

\section{cancer}

Kleppe M, Kraima AC, Kruitwagen RFPM, Van Gorp T, Smit NN, van Munsteren JC,

DeRuiter MC

International Journal of Gynecological Cancer 2015;25:1405-1414 


\begin{abstract}
Objective

In ovarian cancer, detection of sentinel nodes is an upcoming procedure. Peroperative determination of the patient's sentinel node(s) might prevent a radical lymphadenectomy and associated morbidity. It is essential to understand the lymphatic drainage pathways of the ovaries, which are surprisingly up till now poorly investigated, to predict the anatomical regions where sentinel nodes can be found. We aimed to describe the lymphatic drainage pathways of the human ovaries including their compartmental fascia borders.
\end{abstract}

\title{
Methods
}

A series of three human female foetuses and tissues samples from one human cadaveric specimen were studied. Immunohistochemical analysis was performed on paraffin-

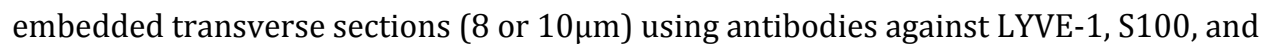
alpha-smooth muscle actin to identify the lymphatic endothelium, Schwann and smooth muscle cells, respectively. Three-dimensional reconstructions were created.

\section{Results}

Two major and one minor lymphatic drainage pathway from the ovaries were detected. One pathway drained via the proper ligament of the ovaries (ovarian ligament) towards the lymph nodes in the obturator fossa and the internal iliac artery. Another pathway drained the ovaries via the suspensory ligament (infundibulopelvic ligament) towards the para-aortic and paracaval lymph nodes. A third minor pathway drained the ovaries via the round ligament to the inguinal lymph nodes. Lymph vessels draining the fallopian tube all followed the lymphatic drainage pathways of the ovaries.

\section{Conclusions}

The lymphatic drainage pathways of the ovaries invariably run via the suspensory ligament (infundibulopelvic ligament) and the proper ligament of the ovaries (ovarian ligament), as well as through the round ligament of the uterus. As ovarian cancer might spread lymphogenously via these routes, the sentinel node can be detected in the paraaortic and paracaval regions, obturator fossa and surrounding internal iliac arteries, and 
inguinal regions. These findings support the strategy of injecting tracers in both ovarian ligaments to identify sentinel nodes. 


\section{Introduction}

Epithelial ovarian cancer has the highest mortality rate of all gynaecological malignancies. This is partially because $75 \%$ of patients are diagnosed with an advanced stage of epithelial ovarian cancer. In patients with clinical early-stage epithelial ovarian cancer, a surgical staging procedure is recommended, which includes total abdominal hysterectomy with bilateral salpingo-oöphorectomy, omentectomy and numerous peritoneal biopsies. Comprehensive lymph node sampling forms an essential part in determining the clinical stage of epithelial ovarian cancer. The International Federation of Obstetrics and Gynaecology (FIGO) recommends complete pelvic and para-aortic lymphadenectomy; however, the extent of lymph node dissection differs greatly from centre to centre. ${ }^{1-3}$ The more lymph nodes removed, the higher the chance of detecting metastases. ${ }^{4}{ }^{5}$ Complete pelvic and para-aortic lymphadenectomy has shown to detect up to 250 lymph nodes. ${ }^{6}$ Systematic lymphadenectomy is considered the gold standard as positive lymph nodes can be missed by lymph node sampling. However, radical lymphadenectomy is associated with serious morbidity. 5, 7,8 The sentinel node procedure has proven to be effective in vulvar, cervical and endometrial cancer. ${ }^{9}$ Recently, a feasibility study has shown that detection of sentinel nodes in ovarian cancer is safe and promising by injecting tracers in the ovarian ligaments. ${ }^{7}$ To optimise the sentinel node procedure in ovarian cancer, excellent understanding of the routes for lymphatic spread is essential. Up to now, studies reporting the lymphatic drainage pathways of the ovaries on microscopic level are lacking.

Epithelial ovarian cancer can metastasize intraperitoneally (in the peritoneal cavity), lymphogenously, and haematogenously. ${ }^{10,11}$ In terms of lymphogenous spread, lymphatic metastases of epithelial ovarian cancer occur mainly in the para-aortic and paracaval lymph nodes. ${ }^{12}$ It is believed that tumour cells follow the lymph vessels accompanying the ovarian artery and vein in the suspensory ligament of the ovary (also named the infundibulopelvic ligament) up to the high para-aortic region and paracaval region. However, pelvic lymph node metastases are also frequent.4-6,13-21 These tumour cells probably follow a different route, possibly along the para-uterine vessels in the broad ligament of the uterus (ligamentum latum) towards the uterine artery and vein and eventually to the iliac vessels. Some case reports have also described isolated inguinal node metastases of epithelial ovarian cancer.5, 22-24 The exact mechanism of this route of 
metastasis is still unclear, but cells may follow the round ligament of the uterus (also named the ligamentum teres uteri) or may follow the external iliac vessels.

Immunohistochemistry is an excellent tool to study lymphatic drainage pathways on a microscopic level. The human lymphatic system develops shortly after the blood vasculature. By the end of the fifth embryonic week, lymph sacs arise from developing veins. These lymph sacs and plexuses lose their venous connection and progress to the peripheral regions as they fuse with another. By the end of the embryonic period $\left(11^{\text {th }}\right.$ week) the formation of new plexuses has stopped, and major definitive lymphatic pathways can be identified. ${ }^{25}$

The objective of this study was to analyse immunohistochemically and to reconstruct three-dimensionally the lymphatic drainage pathways of the ovaries in human foetal pelves. Implications of these findings for the surgical management of epithelial ovarian cancer, including detection of sentinel nodes, will also be discussed.

\section{Materials and methods}

\section{Material}

Three human female foetuses with an embryonic age of 14, 15, and 20 weeks, respectively, from the Department of Anatomy and Embryology, Leiden University Medical Centre and the University of Warsaw in Poland were studied. ${ }^{25}$ Foetal tissues allow accurate exploration of the 3D anatomy of the human pelvic lymphatic system. The foetus of 20 weeks suffered from a trisomy 14 with a terminal $5 p$ depletion. The other foetuses were phenotypically normal and did not show congenital macroscopic malformations. All foetuses were obtained with informed consent after miscarriage or legal abortion. The patients were informed that the foetuses were used for medical research. In the foetuses of 14 and 15 weeks, the bony pelvis was manually removed. The foetus of 20 weeks was decalcified in a $10 \%$ ethylenediaminetetraacetic acid (EDTA) solution for 72 hours. An additional human cadaveric specimen was used to study the round ligament of the uterus, the suspensory ligament of the ovary (infundibulopelvic ligament), and the proper ligament of the ovary (ovarian ligament). This cadaver was fixed in Fix 4 Life solution facilitating subsequent immunohistochemical analysis. This concerned a person who 
during life decided by means of a written consent to donate her body, after death, to medical science.

\section{Immunohistochemistry}

All tissues were embedded in paraffin blocks. Serial transverse sections of 8 and $10 \mu \mathrm{m}$ were produced and stained with haematoxylin and eosin, or with azan. Additionally, series of selected sections were stained with antibodies against Lyve-1 (ReliaTech), S100 (DAKO), and $\alpha$-Smooth Muscle Actin (SMA; Sigma-Aldrich). We used Lyve-1 to visualise lymphatic endothelial cells, S100 to stain Schwann cells in order to reveal the peripheral neural network, and SMA to detect vascular and visceral smooth muscle fibres. Lyve-1 and SMA were incubated on consecutive sections to distinguish between blood and lymph vessels. The sections were rinsed twice with phosphate-buffered saline (PBS) and once with PBS/0.05\% Tween-20 between all steps, unless otherwise indicated. After deparaffinisation and rehydration in graded ethanol dilutions, antigen retrieval was performed by heating the sections $\left(12\right.$ minutes to $\left.98^{\circ} \mathrm{C}\right)$ in citric acid buffer $(0.01 \mathrm{Mol} / \mathrm{L}$, pH 6.0). Then, sections were incubated for 20 minutes in PBS with $0.3 \% \mathrm{H}_{2} \mathrm{O}_{2}$ to inhibit endogenous peroxidase. Sections were incubated overnight with primary antibodies to Lyve-1 $(1 / 200 \mu \mathrm{L})$, S100 $(1 / 5.000 \mu \mathrm{L})$, or SMA $(1 / 5.000 \mu \mathrm{L})$ diluted in a $1 \%$ PBSBSA/Tween-20 solution. The next day, sections were incubated for 45 minutes with a biotin-labelled secondary antibody (goat-anti-rabbit, BA-1000, Vector Labs) and additionally incubated for 45 minutes with the Vectastain ABC staining kit (PK-6100, Vector Labs). Sections stained with SMA were incubated only with a secondary antibody (rabbit-anti-mouse, P-0260, DAKO). Before visualisation, all sections were rinsed twice with PBS and once with Tris/Maleate (pH 7.6). Visualisation was performed with 33'diaminobenzidin tetrahydrochloride as a chromogen, and haematoxylin was used as a counterstain. All sections were mounted with Entellan (1.07961.0100, Merck).

\section{Image processing}

3D reconstructions were generated showing the ovarian lymphatic drainage pathways and autonomic nerves of the foetus aged 14 weeks and the ovarian lymphatic pathways of the foetus aged 15 weeks. With an Olympus AX70-microscope and Olympus D12 camera (Olympus, Tokyo, Japan), micrographs were made. Every $20^{\text {th }}$ section was used for the reconstruction, comprising the entire foetal pelvis up to the upper limit of the ovaries. 
This created a cross-sectional interval of $160 \mu \mathrm{m}$. The Amira ${ }^{\circledR}$ software package version 5.3.3 (Template Graphics Software; Visage Imaging, San Diego, California, USA) was used for the 2D labelling of anatomical structures. The DeVIDE software was used to assemble the 3D volume and to reconstruct the 3D surface meshes ${ }^{26}$. DAZ studio version 4.6 (DAZ 3D, Salt Lake City, USA) was used to develop an interactive PDF file in which the 3D models could be explored.

\section{Results}

Lymph vessels were detected by Lyve-1 positivity in the lymphatic endothelium and by the absence of smooth muscle fibres and red blood cells. In all foetuses, the fallopian tubes and ovaries were situated longitudinally in the intra-abdominal cavity rather than transversally as a consequence of the still developing female genital tract. In addition, the position of the fallopian tubes and ovaries remained relatively high in the abdomen. All results can be visualised three-dimensionally online at: (https://graphics.tudelft.nl/lymphaticdrainageovaries).

The ovaries drained via three routes. The first identified route ran cranially from the ovaries. Lymph vessels draining the ovaries at its cranial part were seen in the suspensory ligament of the ovary (infundibulopelvic ligament) following the course of the ovarian artery (Figure 1 and supplementary file 1). This pathway will be referred to as the abdominal pathway. Lymph vessels accompanied the ovarian artery as it entered the retroperitoneal space and travelled along the common iliac artery and aorta on the left side, and the common iliac artery and caval vein on the right side (Figure 2). Over a certain length of this route, the suspensory ligament of the ovary (infundibulopelvic ligament) was attached to the retroperitoneum, but no lymph vessels from the abdominal pathway were seen to cross the retroperitoneum before the ovarian artery. As a consequence, no connections were seen between the lymph vessels draining the ovaries and the lymph plexuses surrounding the external iliac artery at this level. 
Figure 1. Lymph vessels draining the ovary via the suspensory ligament of the ovary.
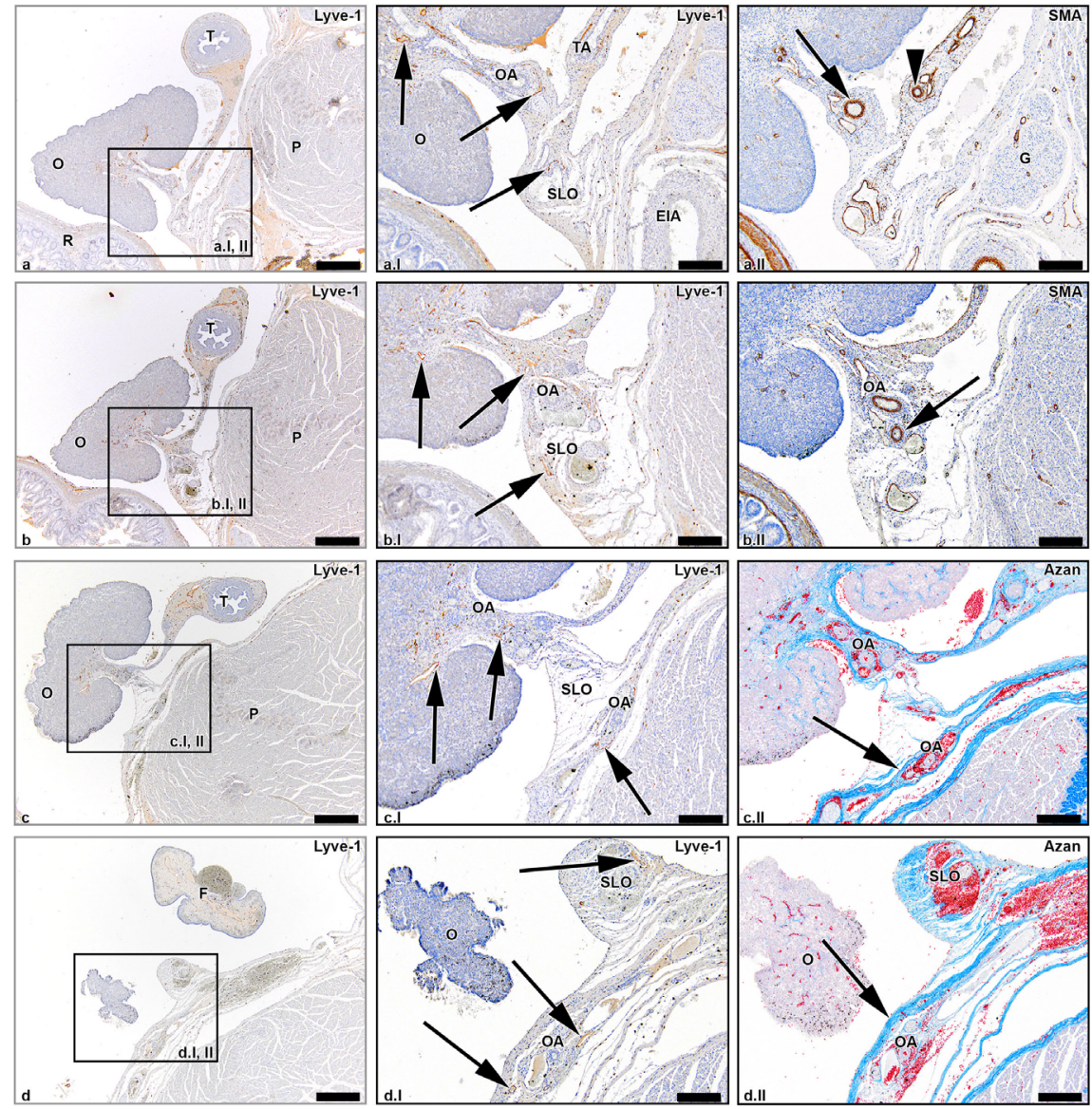

Transverse sections of the foetus aged 15 weeks showing consecutive superior levels ( $a, b, c$ and d). The black arrows in the LYVE-1 stained sections point out lymph vessels draining the ovary $(0)$ via the suspensory ligament of the ovary (SLO) running along the ovarian artery (OA). The arrowhead in window a.II shows the tubal branch of the ovarian artery (TA). The superior part of the OA courses in the SLO (arrow window b.II), whilst the inferior part of the $\mathrm{OA}$ runs in the mesovarium. The superior part of the $\mathrm{OA}$ continues to run in the retroperitoneal compartment (arrows in azan-stained windows). Some lymph vessels remain in the distal part of the SLO (upper arrow window d.I), whereas the major lymph vessels follow the course of the $\mathrm{OA}$ (lower two arrows window d.I). The arrow in window d.II points out the retroperitoneum. T: uterine tube, G: genital branch of genitofemoral nerve, EIA: external iliac artery, P: psoas major muscle, F: fimbriae. Scale bar overview $500 \mu \mathrm{m}$; detail $200 \mu \mathrm{m}$. 
Supplementary file 1. 3D reconstruction of the abdominal pathway.

a

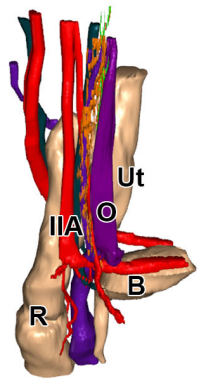

b

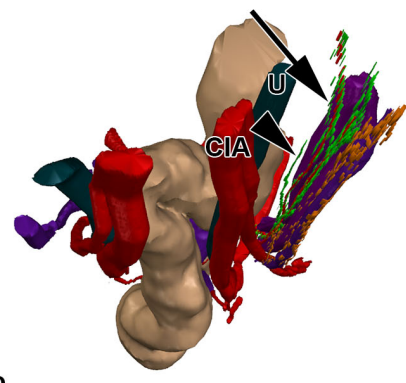

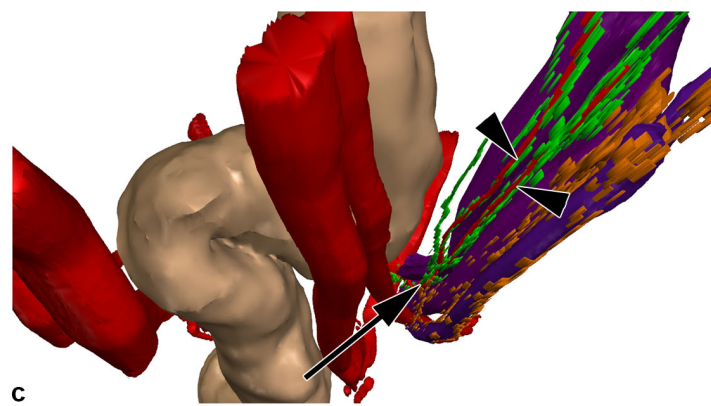

C

A 3D reconstruction of the foetus aged 15 weeks showing the abdominal pathway. Note the longitudinal position of the ovary (window a). Minor lymph vessels draining the ovary (depicted in green) remain in the distal part of the SLO (arrowhead in window b), while major lymph vessels run along the ovarian artery in the proximal part of the SLO (arrow in window b). Lymph vessels draining the uterine tube (depicted in orange) flow into the drainage pathways of the ovary (arrow in window c). The right arrowhead shows the branch of the ovarian artery located in the mesovarium and the left arrowhead the ovarian artery. R: rectum, B: bladder, Ut: uterus, CIA: common iliac artery, U: ureter. 
Figure 2. Suspensory ligament of the ovary and retroperitoneum.
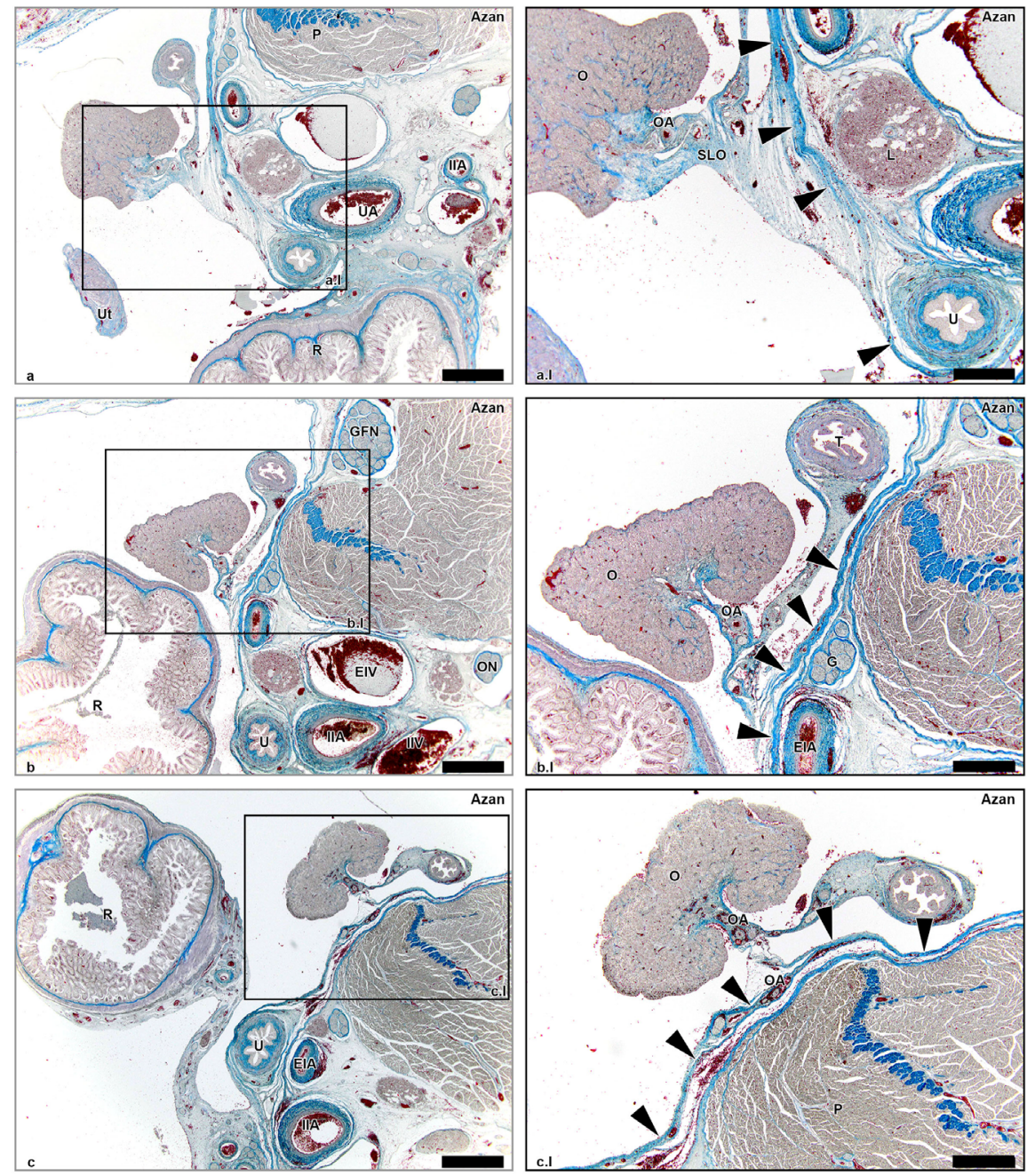

Transverse azan stained sections of the foetus aged 15 weeks showing collagen (blue) and cell nuclei

(purple) at consecutive superior levels ( $a, b$ and $c$ ) The suspensory ligament of the ovary (SLO) does not form a direct continuity with the retroperitoneum as the peritoneum (arrowheads) forms a solid border. Lymph vessels draining via the SLO do not cross this border, but run along the ovarian artery (OA) and shift to the retroperitoneal compartment when the OA does (window c.I). P: psoas major muscle, Ut: uterus, UA: umbilical artery, IIA: internal iliac artery, R: rectum: 0: ovary, U: ureter, EIA: external iliac artery, L: lymph nodes along the EIA, GFN: genitofemoral nerve, EIV: external iliac vein, ON: obturator nerve, IAV: internal iliac vein, T: uterine tube, G: genital branch of GFN. Scale bar overview $1 \mathrm{~mm}$; scale bar detail $500 \mu \mathrm{m}$. 
A second lymphatic drainage pathway ran from the caudal part of the ovaries downwards to the proper ligament of the ovary (ovarian ligament). These lymph vessels followed the ovarian artery that anastomosed with a branch of the uterine artery, the so-called ovarianuterine branch (Figure 3, supplementary file 2). We will refer to this pathway as the pelvic pathway. Lymph vessels accompanying this branch of the uterine artery were detected along the whole corpus uterus (supplementary file 2). At the level of the cervix of the uterus, where the branch of the uterine ovarian artery entered the uterine artery, the lymph vessels continued to follow the uterine artery. In the lateral parametrium, the lymph vessels from this pelvic pathway drained into the supra-ureteral pathway that ran along the course of the uterine artery, crossing the ureter superiorly. The supra-ureteral pathway drained into the lymph plexuses surrounding the internal iliac artery and obturator fossa. 
Figure 3. Lymph vessels draining the ovary via the proper ligament of the ovary and the broad ligament of the uterus.
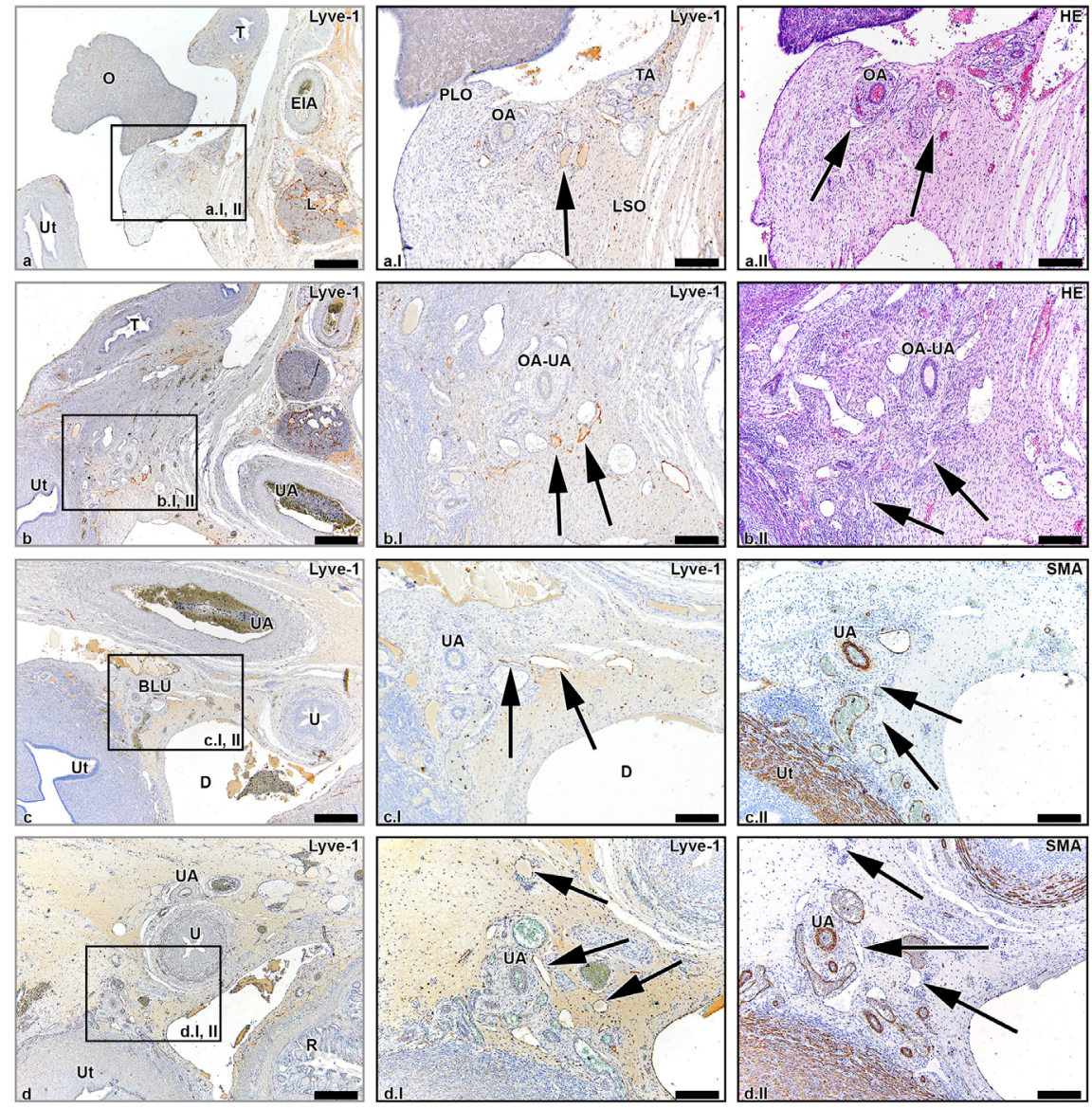

Transverse sections of the foetus aged 14 weeks showing the lymphatic drainage pathway of the ovary (0) via the proper ligament of the ovary (PLO) and broad ligament of the ovary (BLO) at consecutive inferior levels (a, b, c and d). The arrows in the LYVE-1 stained sections show the lymph vessels running along the ovarian artery $(\mathrm{OA})$, merging eventually with the supra-ureteral pathway that follows the course of the uterine artery (UA). Ut: uterus, T: uterine tube, EIA: external iliac artery, L: lymph nodes along the EIA, TA: tubal branches of OA, SLO: suspensory ligament of the ovary, OA-UA: anastomosis of OA and UA, R: rectum, U: ureter, D: Douglas' pouch. Scale bar overview $500 \mu \mathrm{m}$; scale bar detail $200 \mu \mathrm{m}$. 
Supplementary file 2. 3D reconstruction of the pelvic pathway.

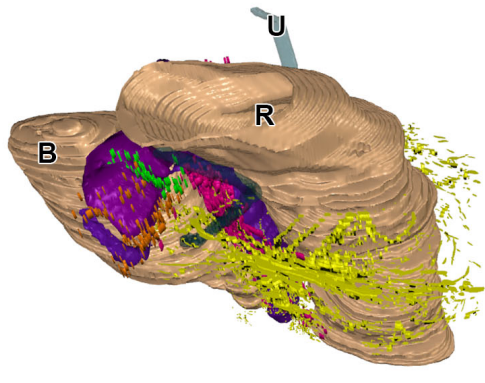

a

b

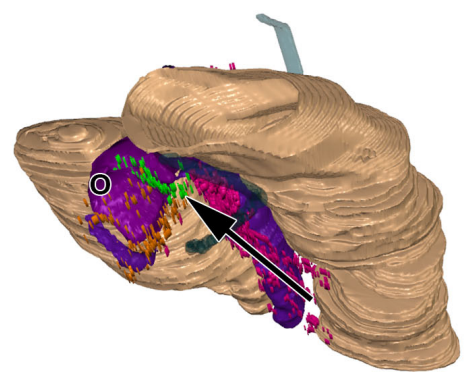

C

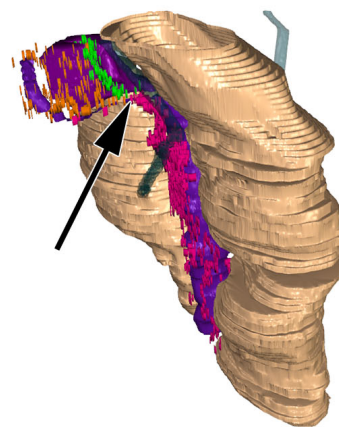

A 3D reconstruction of the foetus aged 14 weeks showing the abdominal pathway. Window a offers a lateral view of the foetal pelvis. Lymph vessels draining the ovary (depicted in green) are located in the mesovarium and lymph vessels draining the uterine tube (depicted in orange) are situated along the uterine tube. Lymph vessels draining the cervix uteri are depicted in magenta. The pelvic autonomic network is depicted in yellow. The arrow in window b indicates the connection between lymph vessels draining the ovary and lymph vessels draining the uterine tube. Window c shows a posterior view in which the connection between lymph vessels draining the ovary and lymph vessels draining the cervix uteri can be explored. B: bladder, R: rectum, U: ureter, 0: ovary.

Besides lymphatic drainage via the abdominal and pelvic pathways, a third minor lymphatic drainage pathway was detected to run in the round ligament of the uterus (Figure 4). This pathway is termed the inguinal pathway. The number of lymph vessels seen in the round ligament of the uterus was small in comparison to the number of lymph vessels in the proper ligament of the ovary (ovarian ligament) and suspensory ligament of the ovary (infundibulopelvic ligament). This pathway was not bilaterally present in all foetal specimens and appeared more prominently in some specimens than in others. In the foetus of 20 weeks, this pathway was less prominent than in the foetuses of 14 and 15 weeks. In the foetus of 15 weeks, the lymph vessels were seen on both sides, although the vessels abruptly disappeared on the left side when travelling more caudally. The vessels that did not disappear became thinner towards the inguinal region and eventually drained into inguinal lymph nodes. 
Figure 4. Lymph vessels draining the ovary via the round ligament of the uterus.
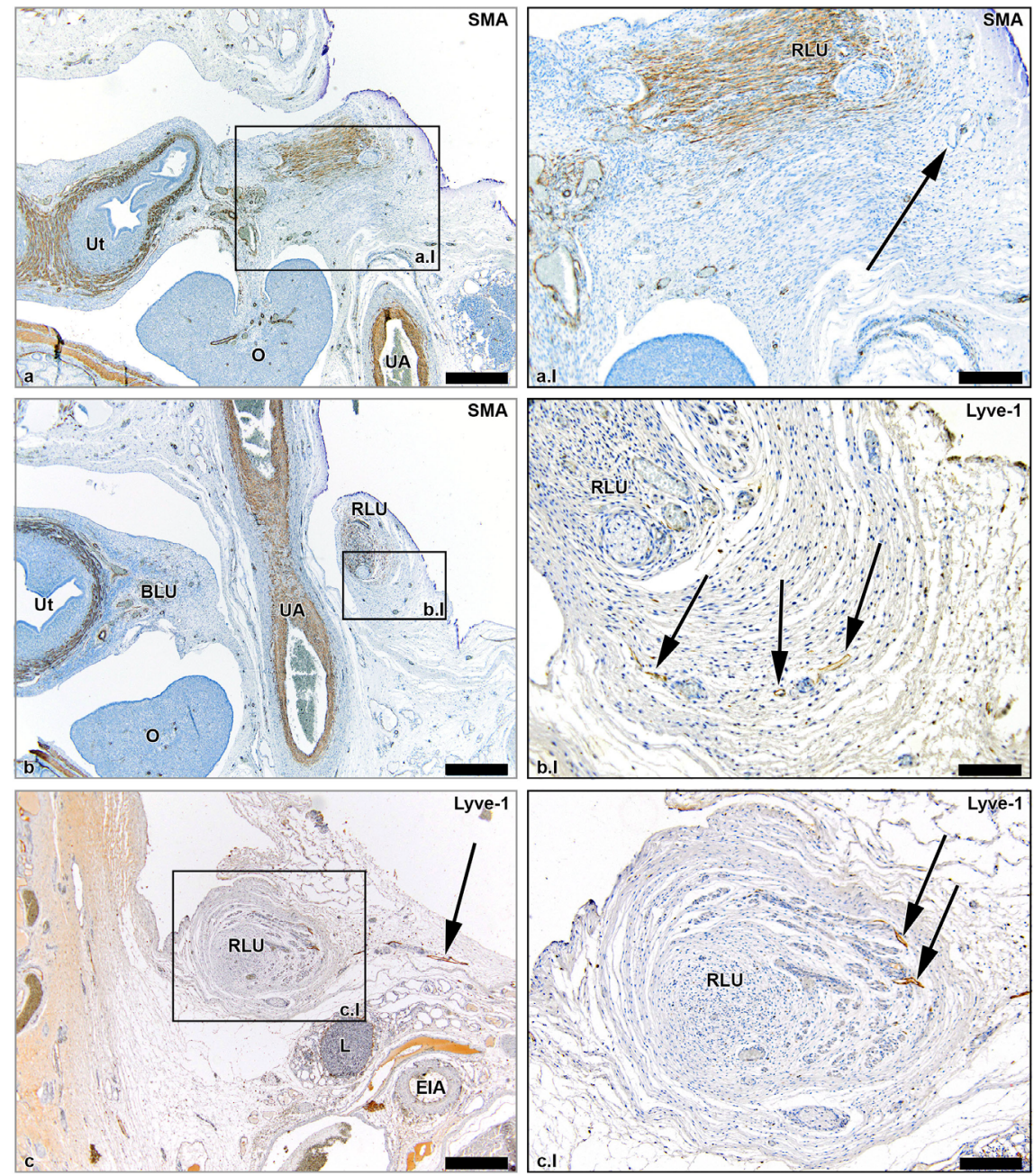

Transverse sections of the foetus aged 14 weeks showing lymph vessels (arrows) that drain the ovary (0) via the round ligament of the uterus (RLU, ligament teres uteri) at consecutive inferior levels ( $a, b$ and $c)$. Note the presence of smooth muscle fibres in the RLU (brown stain in the SMA stained sections). At an inferior level, the RLU passes under the umbilical artery (UA) as can be seen in window b. The RLU runs to the inguinal region and its lymph vessels eventually fuse with inguinal lymph vessels. Ut: uterus, 0: ovary, EIA: external iliac artery, L: lymph nodes along the EIA. Scale bar overview $500 \mu \mathrm{m}$; scale bar detail $200 \mu \mathrm{m}$. 
A considerable number of lymph vessels were seen in the mesovarium along the entire length of the ovary, as well as surrounding the fallopian tube in the mesosalpinx (Figure 5). The lymph vessels in the mesosalpinx drained into all three lymphatic drainage pathways of the ovaries. Over the entire length of the fallopian tube up until the fimbriae, prominent lymphatic drainage was visible. There was no connection detected between the left and the right ovarian pathways.

Figure 5. Lymph vessels draining the uterine tubes.
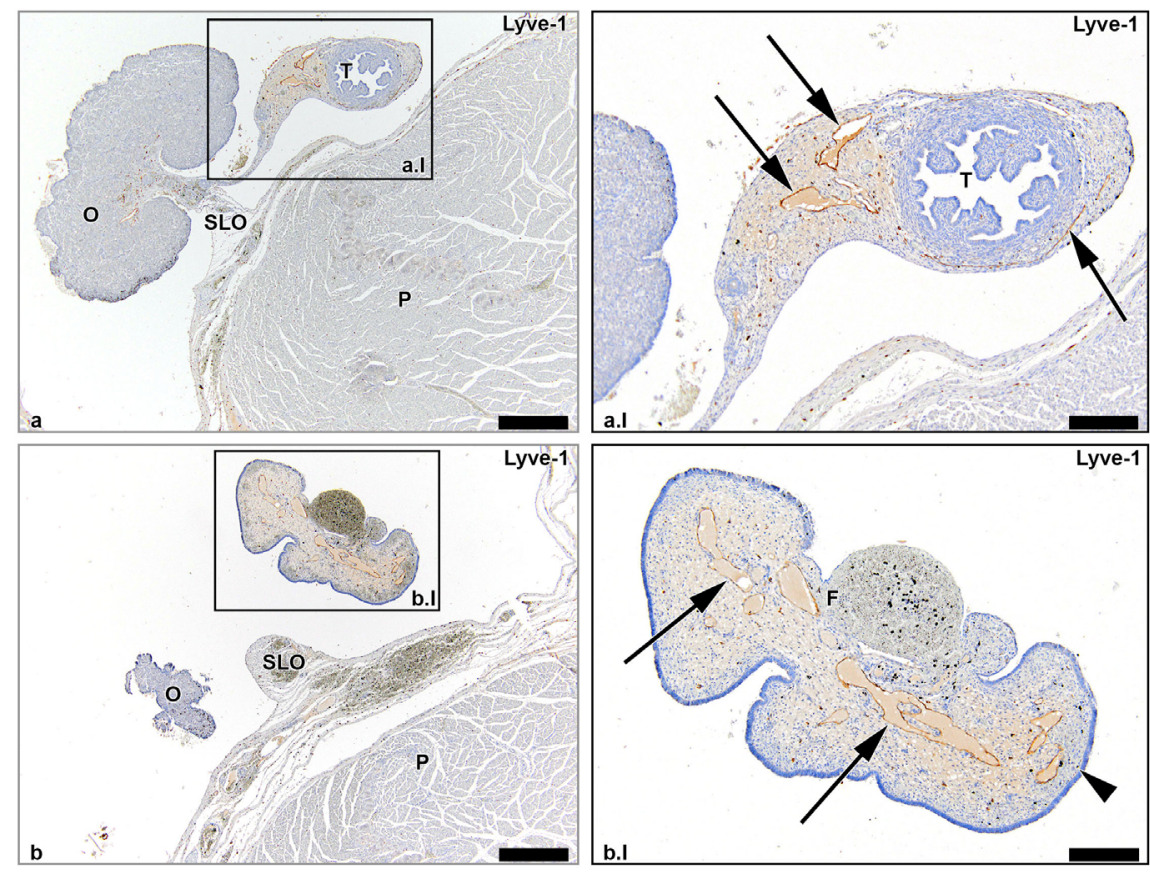

Transverse LYVE-1 stained sections of the foetus aged 15 weeks showing that lymph vessels draining the uterine tubes (arrows) run in the mesosalpinx. Note the substantial amount of lymph vessels along the entire length of the uterine tubes until its fimbriae (F). O: ovary, T: uterine tube, SLO: suspensory ligament of the ovary, P: psoas major muscle. Scale bar overview $500 \mu \mathrm{m}$; scale bar detail $200 \mu \mathrm{m}$.

We confirmed the presence of lymph vessels in the suspensory ligament of the ovary (infundibulopelvic ligament), proper ligament of the ovary (ovarian ligament), and round ligament of the uterus of the human adult cadaveric tissues samples (Figure 6). 
Figure 6. Lymph vessels in the human adult RLU, SLO and PLO.
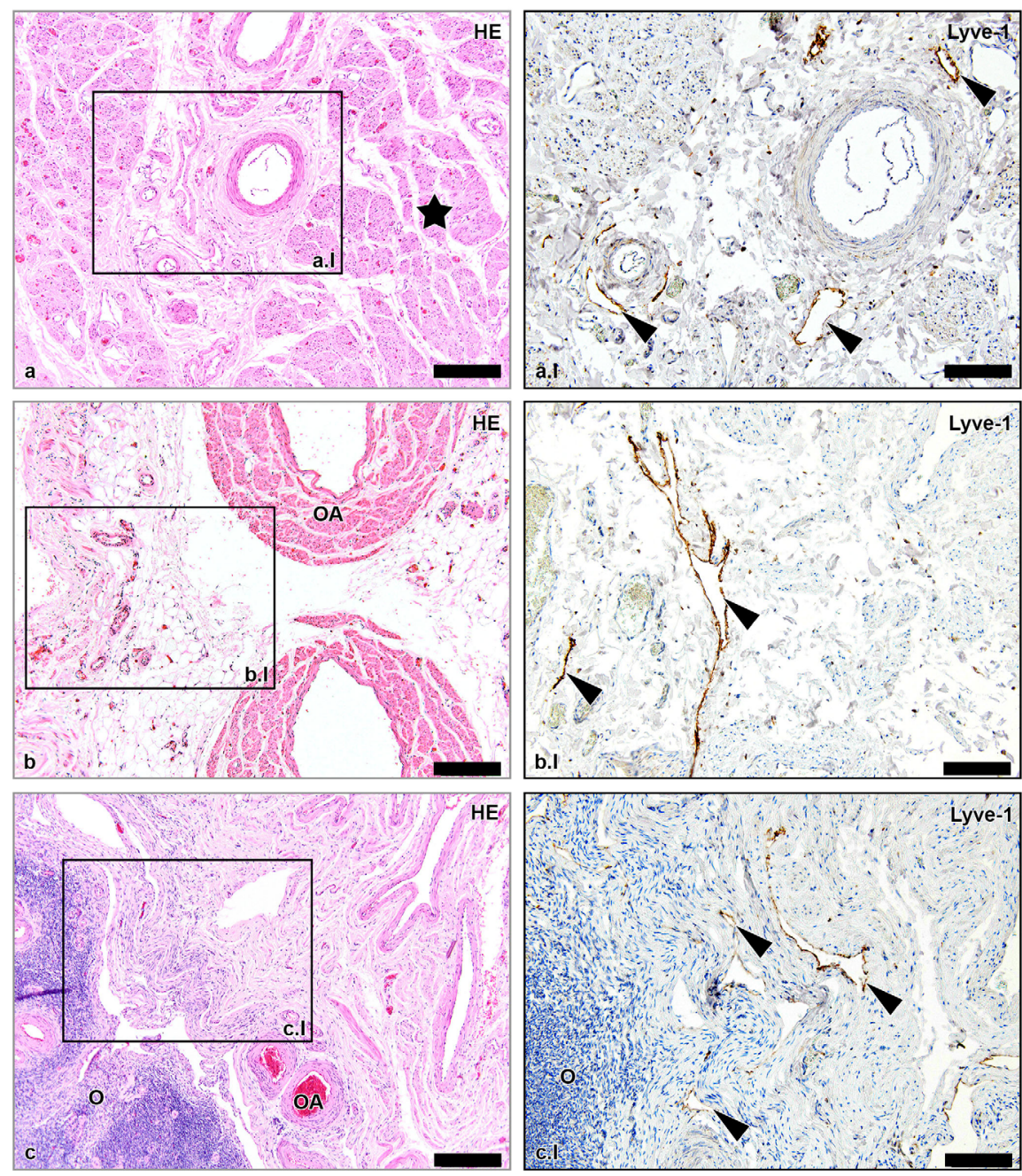

HE stained sections of tissue samples from human adult round ligament of the uterus (RLU; window a), suspensory ligament of the ovary (SLO; window b) and proper ligament of the ovary (PLO; window c). Detail windows a.I, b.I and c.I are LYVE-1 stained adjacent sections revealing the presence of lymph vessels in the three ligaments (arrowheads). The star shows the presence of smooth muscle fibers in the RLU. OA: ovarian artery, 0: ovary. Scale bar overview $200 \mu \mathrm{m}$; scale bar detail $100 \mu \mathrm{m}$. 


\section{Discussion}

So far, few in vivo studies focussed on revealing the lymphatic drainage pathways of the ovaries, but histological studies are lacking. To our knowledge, we are the first to have studied the lymphatic drainage pathways of the ovaries on microscopic level. The sentinel node procedure is an upcoming technique to pre-operatively determine the nodal status in patients suffering from epithelial ovarian cancer. Excellent knowledge of the lymphatic drainage pathways of the ovaries is essential to predict the routes for lymphatic spread and consequently, the sites where sentinel nodes can be detected. Unlike in other sentinel node procedures, such as in breast cancer and malignant melanoma, injection of tracers cannot be done in the skin near the tumour. In addition, injection in the tumour itself should be avoided since this can cause spillage of tumour cells. The ovarian ligaments are therefore a good alternative site for injection of the tracers. ${ }^{7}$

We identified two major and one minor lymphatic drainage pathways of the ovaries: first the abdominal pathway, running via the suspensory ligament of the ovary (infundibulopelvic ligament) to the para-aortic (left side)/para-caval (right side) regions, second the pelvic pathway, draining via the lateral parametrium and supra-ureteral pathway ${ }^{25}$ to the internal iliac artery and obturator fossa, and third the inguinal pathway, draining via the round ligament of the uterus to the inguinal regions. Sentinel nodes can be found in any of these regions. Besides, the findings reflect the most common locations of lymph node metastases in patients with ovarian cancer; $50 \%$ of patients have metastasis solely in the high para-aortic/paracaval area, while $20 \%$ have metastasis solely in the pelvic area and $30 \%$ in both the para-aortic/paracaval and pelvic area. ${ }^{2-6,10-24,27}$ Some rare cases of isolated inguinal metastases have been described in patients with ovarian cancer.5, 22-24 The inguinal pathway as studied in the three foetal specimens significantly diminished when travelling further away from the uterus. This pathway was also less prominent in the foetus of 20 weeks. A potential explanation for this finding is that this pathway disappears during embryologic development in the majority of female foetuses, but in a small percentage of women some lymph vessels may persist forming a route for lymphatic spread of ovarian cancer. Remarkably, the lymphatic drainage pathways of the ovaries via the proper ligament of the ovary (ovarian ligament), suspensory ligament of the ovary (infundibulopelvic ligament), and round ligament of the uterus all arise from the same embryologic structure called the gubernaculum. 
Accurate knowledge of the anatomy and embryology of the human ovaries is essential to understand the patterns of lymphatic metastases in ovarian cancer. In embryos, the mesonephric (Wolffian) and paramesonephric (Müllerian) ducts are discrete primordia that coexist during the ambisexual period of development (until 8 weeks). Thereafter, one duct system persists, while the other regresses, leaving only non-functional vestiges. The Wolffian duct develops first, differentiates into the epididymis, vas deferens, and seminal vesicles in men and regresses in women (in the sixth or seventh week). The Müllerian duct develops later and differentiates into the fallopian tubes, uterus, and upper portion of the vagina. $^{28}$ The ovaries develop at the level of the thoracic vertebra 12 and descend towards the sacral region. Two bands secure the gonads at the lower and upper poles, forming a structure called the gubernaculum. After atrophy of the Müllerian ducts, the upper gubernaculum disappears in men, while the suspensory ligament of the ovary (infundibulopelvic ligament) is formed in women, extending from the upper pole of the ovary upwards towards the lumbar region. The lower part of the gubernaculum inserts into the lower part of the gonad. In women, the proper ligament of the ovary (ovarian ligament) and the round ligament of the uterus are formed. ${ }^{29}$ The minor inguinal pathway cannot be explained by embryological development. However, the presence of lymph vessels in human cadaveric tissue samples suggests that the inguinal pathway is a definitive drainage pathway and may act as a route for lymphatic spread of epithelial ovarian cancer.

Although very rare, contra-lateral metastases have been found in patients with ovarian cancer. ${ }^{27}$ Therefore, guidelines advise that lymphadenectomy should always be performed on both sides, even in cases of a unilateral tumour. ${ }^{27}$ In this study, we did not detect connections between the right and left lymphatic ovarian drainage pathways in the analysed foetuses. A possible explanation for contra-lateral metastases could be retrograde metastasis from one side to the other at the level of the cervix. ${ }^{30,31}$ However, we cannot support this theory based on our findings.

Additionally, a large number of lymph vessels were seen in the ovaries as well as in the mesosalpinx of the fallopian tubes. The fact that all of these lymph vessels drain via two major pathways located in the ovarian ligaments supports the strategy of identifying the sentinel node in ovarian cancer by injecting tracers into the ovarian ligaments. ${ }^{7}$ 
The theory on the origin of epithelial ovarian cancer has changed in recent years. Traditionally, epithelial ovarian cancer was thought to arise from damaged ovarian epithelium. However, recently, studies suggest the fallopian tube as the source of epithelial ovarian cancer, since a precursor of high-grade carcinoma can be found in the uterine tube (serous tubal intra-epithelial carcinoma, STIC). ${ }^{32}$ The fimbriae, which are in close contact with the ovaries, are the preferred site of STIC. As the lymph vessels detected in the mesosalpinx eventually drain into ovarian pathways, irrespective of the exact origin of epithelial ovarian cancer (uterine tubes or ovaries), the locations where the lymphogenous metastases can be found are the same.

The study of the lymphatic system in foetuses has some limitations. ${ }^{25}$ As LYVE-1 also recognizes venous endothelial cells in very early stages. Therefore, comparative immunohistochemical studies on adult specimens using LYVE-1 are needed to understand its staining patterns in developed tissues. In this study, we used human adult cadaveric tissue samples as control tissue to assess the presence of lymph vessels in the suspensory ligament of the ovary (infundibulopelvic ligament), proper ligament of the ovary (ovarian ligament), and round ligament of the uterus. Although it was not feasible to study complete lymphatic drainage pathways in these tissues, we confirmed the presence of lymph vessels in all ligaments. This supported our findings in the human foetal specimens. Despite the relatively low number of foetal specimens studied, we found three consistent pathways of lymphatic drainage. Definitive patterns from the human lymphatic network can be recognized from embryonic 12 weeks onwards. However, significant changes may occur in later developmental stages or under pathological circumstances. Therefore, future studies that focus on the lymphatic drainage pathways of the ovaries in the human adult are needed. Finally, foetal ovaries are still located relatively high within the abdomen compared to adult ovaries. The ovaries descend slightly during embryologic development, but not as much as the testis in men. Nevertheless, while these organs may change position during embryological development, the vasculature and lymphatic drainage remains the same. 


\section{Conclusion}

Epithelial ovarian cancer can metastasize via three lymphatic drainage pathways. The abdominal pathway travels through the suspensory ligament of the ovary (infundibulopelvic ligament) along with the ovarian artery towards the aorta (right side) and caval vein (left side), while the pelvic pathway runs via the proper ligament of the ovary (ovarian ligament) along with the uterine artery towards the iliac artery and obturator fossa. Metastasis can also occur in the inguinal region as a result of a third minor lymphatic drainage pathway running via the round ligament of the uterus. Sentinel nodes can be detected in any of these regions.

\section{Acknowledgments}

We like to thank Andrev Kolesnik from the University of Warsaw in Poland for providing us with two human foetal pelves. 


\section{References}

1. Benedet JL, Bender H, Jones H, $3^{\text {rd }}$, Ngan HY, Pecorelli S. FIGO staging classifications and clinical practice guidelines in the management of gynecologic cancers. FIGO Committee on Gynecologic Oncology. Int J Gynaecol Obstet 2000;70:209-262.

2. Angioli R, Plotti F, Palaia I, Calcagno M, Montera R, Cafà EV, Sereni MI, Panici PB. Update on lymphadenectomy in early and advanced ovarian cancer. Curr Opin Obstet Gynecol 2008;20:34-39.

3. Di Re F, Baiocchi G. Value of lymph node assessment in ovarian cancer: status of the art at the end of the second millennium. Int J Gynecol Cancer 2000;10:435-442.

4. Maggioni A, Benedetti Panici P, Dell'Anna T, Landoni F, Lissoni A, Pellegrino A, Rossi RS, Chiari S, Campagnutta E, Greggi S, Angioli R, Manci N, Calcagno M, Scambia G, Fossati R, Florani I, Torri V, Grassi R, Mangioni C. Randomized study of systematic lymphadenectomy in patients with epithelial ovarian cancer macroscopically confined to the pelvis. Br J Cancer 2006;18:699-704.

5. Carnino F, Fuda G, Ciccone G, Iskra L, Guercio E, Dadone D, Conte PF. Significance of lymph node sampling in epithelial carcinoma of the ovary. Gynecol Oncol 1997;65:467-472.

6. Harter P, Gnauert K, Hils R, Lehmann TG, Fisseler-Eckhoff A, Traut A, du Bois A. Pattern and clinical predictors of lymph node metastases in epithelial ovarian cancer. Int J Gynecol Cancer 2007;17:1238-1244.

7. Kleppe M, Brans B, Van Gorp T, Slangen BFM, Kruse AJ, Pooters INA, Lotz MG, Van de Vijver KK, Kruitwagen RFPM. The detection of Sentinel Nodes in Ovarian Cancer: a feasibility study. J Nucl Med 2014;55:1799-1804.

8. Ditto A, Martinelli F, Reato C, Kusamura S, Solima E, Fontanelli R, Haeusler E, Raspagliesi F. Systematic par-aortic and pelvic lymphadenectomy in early stage epithelial ovarian cancer: a prospective study. Ann Surg Oncol 2012;19:3849-3855.

9. Handgraaf HJM, Verbeek FPR, Tummers QRJG, Boogerd LSF, van de Velde CJH, Vahrmeijer AL, Gaarenstroom KN. Real-time near-infrared fluorescence guided surgery in gynecologic oncology: A review of the current state of the art. Gynecol Oncol 2014;135:606-613.

10. Panici PB, Angioli R. Role of lymphadenectomy in ovarian cancer. Best Pract Res Clin Obstet Gynaecol 2002;16:529-551. 
11. Tan DS, Agarwal R, Kaye SB. Mechanisms of transcoelomic metastasis in ovarian cancer. Lancet Oncol 2006;7:925-934.

12. Burghardt E, Girardi F, Lahousen M, Tamussino K, Stettner H. Patterns of pelvic and paraaortic lymph node involvement in ovarian cancer. Gynecol Oncol 1991;40:103106.

13. Benedetti-Panici P, Greggi S, Maneschi F, Scambia G, Amoroso M, Rabitti C, Mancuso S. Anatomical and pathological study of retroperitoneal nodes in epithelial ovarian cancer. Gynecol Oncol 1993;51:150-154.

14. Onda T, Yoshikawa H, Yokota H, Yasugi T, Taketani Y. Assessment of metastases to aortic and pelvic lymph nodes in epithelial ovarian carcinoma. A proposal for essential sites for lymph node biopsy. Cancer 1996;78:803-808.

15. Tsumura N, Sakuragi N, Hareyama H, Satoh C, Oikawa M, Yamada H, Yamamoto R, Okuyama K, Fujino T, Sawaga T, Fujimoto S. Distribution pattern and risk factors of pelvic and para-aortic lymph node metastasis in epithelial ovarian carcinoma. Int J Cancer 1998;79:526-530.

16. Sakuragi N, Yamada H, Oikawa M, Okuyama K, Fujino T, Sagawa T, Fujimoto S. Prognostic significance of lymph node metastasis and clear cell histology in ovarian carcinoma limited to the pelvis (pT1M0 and pT2M0). Gynecol Oncol 2000;79:251255.

17. Suzuki M, Ohwada M, Yamada T, Kohno T, Sekiguchi I, Sato I. Lymph node metastasis in stage I epithelial ovarian cancer. Gynecol Oncol 2000;79:305-308.

18. Morice P, Joulie F, Camatte S, Atallah D, Rouzier R, Pautier P, Pomel C, Lhommé C, Duvillard P, Castaigne D. Lymph node involvement in epithelial ovarian cancer: analysis of 276 pelvic and paraaortic lymphadenectomies and surgical implications. J Am Coll Surg 2003;197:198-205.

19. Negishi H, Takeda M, Fujimoto T, Todo Y, Ebina Y, Watari H, Yamamoto R, Minakami H, Sakuragi N. Lymphatic mapping and sentinel node identification as related to the primary sites of lymph node metastasis in early stage ovarian cancer. Gynecol Oncol 2004;94:161-166.

20. Takeshima N, Hirai Y, Umayahara K, Fujiwara K, Takizawa K, Hasumi K. Lymph node metastasis in ovarian cancer: difference between serous and non-serous primary tumours. Gynecol Oncol 2005;99:427-431. 
21. Nomura H, Tsuda H, Susumu N, Fujii T, Banno K, Kataoka F, Tominaga E, Suzuki A, Chiyoda T, Aoki D. Lymph node metastasis in grossly apparent stages I and II epithelial ovarian cancer. Int J Gynecol Cancer 2010;20:341-345.

22. Ang D, Ng KY, Tan HK, Chung AY, Yew BS, Lee VK. Ovarian carcinoma presenting with isolated contralateral inguinal lymph node metastasis: a case report. Ann Acad Med Singapore 2007;36:427-430.

23. Oei AL, de Hullu JA, Grefte JM, Havenith BP. An enlarged groin node as first manifestation of a malignancy: don't forget the ovaries. Eur J Obstet Gynecol Reprod Biol 2008;138:240-242.

24. Fournier M, Stoeckle E, Guyon F, Brouste V, Thomas L, MacGrogan G, Floquet A. Lymph node involvement in epithelial ovarian cancer: sites and risk factors in a series of 355 patients. Int J Gynecol Cancer 2009;19:1307-1313.

25. Kraima AC, Derks M, Smit NN, Van Musteren JC, Van der Velden J, De Ruiter MC. Lymphatic drainage pathways from the cervix uteri: Implications for radical hysterectomy? Gynecol Oncol 2014;132:107-113.

26. Botha CP, Post FH. Hybrid scheduling in the DeVIDE dataflow visualisation environment. SCS Publishing House Erlangen 2008:309-322.

27. Kleppe M, Wang T, Van Gorp T, Slangen BFM, Kruse AJ, Kruitwagen RFPM. Lymph node metastasis in stages I and II ovarian cancer: a review. Gynecol Oncol 2011;123:610-614.

28. Fritz MA, Speroff L. Clinical gynecologic, endocrinology and infertility. 8th ed. Philadelphia: Lippincott Williams \& Wilkins; 2011.

29. Van der Schoot P, Emmen JMA. Development, structure and function of the cranial suspensory ligaments of the mammalian glands in a cross-species perspective; their possible role in effecting disturbed testicular descent. Human Reprod Update 1996;5:399-418.

30. Eicher E, Bove ER. In vivo studies on the lymphatic drainage of the human ovary. Obstet Gynecol 1954;3:287-297.

31. Eicher E, Goldberg I, Bove ER. In vivo studies with direct sky blue of the lymphatic drainage of the internal genitals of women. Am J Obstet Gynecol 1954;67:12771287.

32. Vang R, Shih IM, Kurman RJ. Fallopian tube precursors of ovarian low- and highgrade serous neoplasms. Histopathology 2013;62:44-58. 


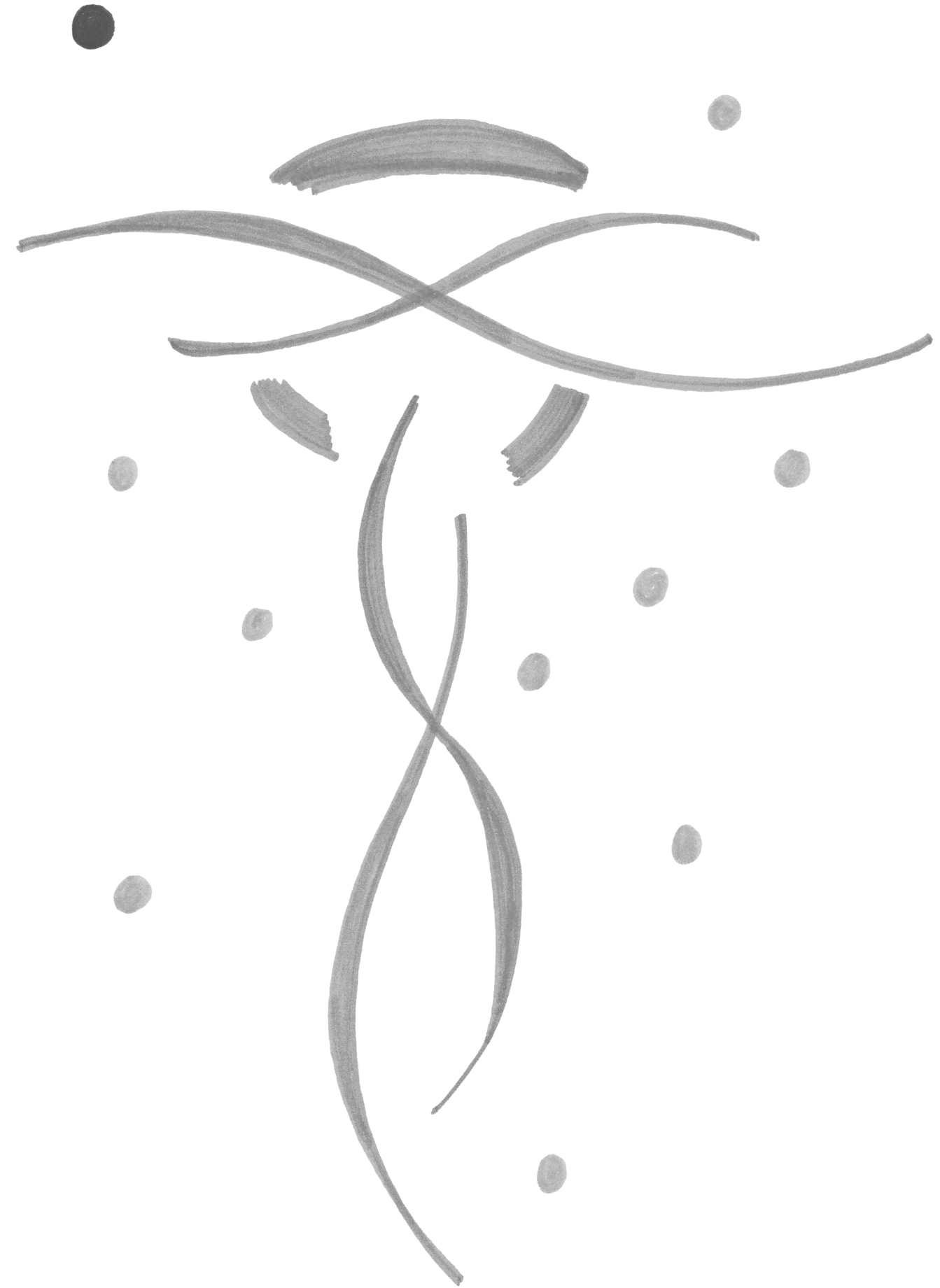




\section{Chapter 7}

\section{The impact of lymph node}

dissection and adjuvant

\section{chemotherapy on survival:}

a nationwide cohort study of patients with clinical

\section{early-stage ovarian cancer}

Kleppe M, van der Aa M, Van Gorp T, Slangen BFM, Kruitwagen RFPM European Journal of Cancer 2016;66:83-90 


\begin{abstract}
Introduction

To establish the impact of lymph node dissection and chemotherapy on survival in patients with early-stage epithelial ovarian cancer (EOC).
\end{abstract}

\title{
Methods
}

All Dutch patients with International Federation of Gynaecology and Obstetrics (FIGO) stage I-IIA and IIIA1 EOC between 2000 and 2012 were included. Data concerning age, stage, tumour-grade, histological subtype, hospital type, lymph node dissection, adjuvant chemotherapy and survival were extracted from the Netherlands Cancer Registry.

\section{Results}

Of 3658 patients included, 1813 (49.6\%) had lymph nodes removed. Relative survival of patients with lymph node dissection (including those with lymph node metastases) was significantly better than that of patients without, also after correcting for stage, tumour-grade, histology and age (89\% and 82\% respectively; relative excess risk (RER) 0.64 ; 95\% confidence interval (CI) 0.52-0.78).

There was a positive correlation between the number of removed lymph nodes and overall survival (after excluding patients with lymph node metastases).

Of patients with stage I-IIA EOC who had $\geq 10$ lymph nodes removed, there was no difference in relative survival between those that received chemotherapy and those that did not ((RER 0.51; 95\% CI 0.15-1.64). This was also true for a subgroup of patients with high-risk features (stage IC and IIA and/or tumour-grade 3 and/or clear cell histology (RER 0.90; 95\% CI 0.46-1.99).

\section{Conclusion}

Adequate dissection of at least 10 , but preferably $\geq 20$ lymph nodes should be standard procedure for the staging of early-stage EOC. Adjuvant chemotherapy after an adequate lymph node dissection does not seem to contribute to a better relative survival. 


\section{Introduction}

Ovarian cancer is the gynaecological tumour with the least favourable prognosis. Five year relative survival ranges from $38-46 \%{ }^{1}$ This is because approximately $70-75 \%$ of patients are diagnosed with advanced stage epithelial ovarian cancer (EOC). For advanced stage disease, there is consensus regarding the importance of both debulking surgery and chemotherapy. By contrast, for early-stage disease, the extent of the surgical staging procedure, especially the lymph node dissection, and the role of adjuvant chemotherapy remain controversial. ${ }^{2,3}$

FIGO recommends complete para-aortic and pelvic lymphadenectomy. ${ }^{4}$ However, lymphadenectomy is a radical procedure associated with morbidities. ${ }^{5}$ Furthermore, some studies indicate that, irrespective of the extent of the staging procedure, adjuvant chemotherapy improves survival in women with early-stage EOC.6-8 Other studies have shown that the benefits of adjuvant chemotherapy are predominantly in patients in who surgical staging was incomplete. 7, 8 Therefore, surgical staging, particularly the extent of lymph node dissection, differs greatly from centre to centre, as do the indications for adjuvant chemotherapy. ${ }^{9}$

The goals of this nationwide cohort study were to establish the impact of lymph node dissection and adjuvant chemotherapy on the survival of patients with early-stage EOC.

\section{Patients and methods}

\section{Study design and patients}

We performed a nationwide retrospective cohort study by analysing data from the Netherlands Cancer Registry (NCR) between $1^{\text {st }}$ January 2000 and $31^{\text {st }}$ December 2012. All patients diagnosed with FIGO stage I-IIA and IIIA1 EOC were included. Patients who did not undergo surgery were excluded, as were patients with borderline tumours.

The NCR is maintained by the Netherlands Comprehensive Cancer Organization and contains data on all cancer patients in the Netherlands. The NCR, which reached full national coverage in 1989, relies on notification of all newly diagnosed malignancies via the automated nationwide pathology archive. Trained registrars use standardized forms to collect patient information from medical records, and regular consistency checks are 
performed to ensure the quality of the data held in the NCR. The completeness of the NCR is estimated to be $95 \% .^{10,11}$ The following data were examined in this study: age, histological subtype, tumour stage, differentiation grade, number of lymph nodes removed, whether or not adjuvant chemotherapy was administered and type of hospital. The date of death was extracted from the municipal population register.

Tumour stage was based on the TNM staging system and defined according to the 2014 FIGO staging system. ${ }^{4}$ The number of removed lymph nodes was categorized as follows: 0 , $1-9,10-19,20-29$ and $\geq 30$. Other elements of staging (i.e., inspection of the abdominal cavity, omentectomy and peritoneal biopsies) were not investigated because these items are not registered in the NCR.

Hospitals were classified according to their level of specialization as follows: specialized, semi-specialized or general. Specialized hospitals are regional centres for gynaecologic oncologic care in which patients are treated by gynaecologic oncologists. Semi-specialized hospitals are usually large community hospitals where patients are treated by gynaecologists with a special interest in gynaecologic oncology. General hospitals are usually smaller hospitals that do not have (semi-) specialized oncologic care.

High-risk tumours were defined as a FIGO stage IC and IIA and/or differentiation grade 3 and/or clear cell histology.

\section{Statistical analysis}

Data analysis was performed using STATA data analysis and statistical software (StataCorp, College Station, TX). Comparisons between groups were performed using the $\chi 2$ test. Overall survival was defined as the interval from the date of diagnosis to the date of death. The Kaplan-Meier method was used to create overall survival curves. Relative survival rates were calculated as an estimation of cause-specific survival, according to the Ederer II method. ${ }^{12}$ Relative survival was calculated as the ratio of observed survival among the cancer patients to expected survival, the expected number of deaths being calculated by national life tables matched for age, sex, and year of incidence. Relative excess risk (RER) of death and $P$ values were estimated by a multivariable generalized forward stepwise linear model with a Poisson distribution based on collapsed relative survival databased on exact survival times. 


\section{Results}

Patient characteristics

In total, 3756 patients with stage I-IIA and IIIA1 invasive EOC were identified. After excluding patients who did not undergo surgery $(\mathrm{n}=98), 3658$ patients were included which characteristics are shown in Table 1.

Table 1. Patients characteristics

\begin{tabular}{|c|c|c|c|c|c|c|c|c|c|c|c|c|c|}
\hline & & \multicolumn{8}{|c|}{ Lymph nodes resected } & \multicolumn{4}{|c|}{ Chemotherapy } \\
\hline & & \multicolumn{2}{|l|}{-} & \multicolumn{6}{|c|}{+} & \multicolumn{2}{|l|}{-} & \multicolumn{2}{|l|}{+} \\
\hline & & \multirow[b]{2}{*}{ Number } & \multirow[b]{2}{*}{$\%$} & \multicolumn{2}{|l|}{$<10$} & \multicolumn{2}{|c|}{$\geq 10$} & \multicolumn{2}{|c|}{ Unknown } & \multirow[b]{2}{*}{ Number } & \multirow[b]{2}{*}{$\%$} & \multirow[b]{2}{*}{ Number } & \multirow[b]{2}{*}{$\%$} \\
\hline & & & & Number & $(\%)$ & Number & $(\%)$ & Number & $(\%)$ & & & & \\
\hline \multirow[t]{2}{*}{ Age } & $<75$ years & 1510 & (47) & 654 & (20) & 664 & $(21)$ & 368 & (12) & 1886 & (59) & 1310 & (41) \\
\hline & $\geq 75$ years & 335 & (72) & 68 & (15) & 33 & (7) & 26 & (6) & 331 & (72) & 131 & (28) \\
\hline \multirow[t]{5}{*}{ Tumour type } & Serous & 506 & (51) & 200 & (20) & 173 & (18) & 110 & (11) & 485 & (49) & 504 & (51) \\
\hline & Mucinous & 452 & (50) & 168 & (18) & 185 & (20) & 108 & (12) & 748 & (82) & 165 & (18) \\
\hline & Endometrioid & 354 & (43) & 187 & (23) & 192 & (24) & 83 & (10) & 493 & $(60)$ & 323 & (40) \\
\hline & Clear cell & 189 & (44) & 98 & (23) & 93 & (21) & 51 & (12) & 139 & (32) & 292 & (68) \\
\hline & Other & 344 & (68) & 69 & (13) & 54 & (11) & 42 & (8) & 352 & (69) & 157 & (31) \\
\hline \multirow[t]{5}{*}{ Tumour differentiation grade } & Well & 400 & (45) & 180 & (20) & 209 & (23) & 107 & (12) & 709 & (79) & 187 & (21) \\
\hline & Moderate & 367 & (44) & 177 & (22) & 185 & (22) & 100 & (12) & 473 & (57) & 356 & (43) \\
\hline & Poor & 386 & (49) & 171 & (22) & 142 & (18) & 83 & (11) & 251 & (32) & 531 & (68) \\
\hline & Undifferentiated & 9 & (36) & 10 & (40) & 1 & (4) & 5 & (20) & 5 & (20) & 20 & (80) \\
\hline & Unknown & 683 & (61) & 184 & (16) & 160 & (14) & 99 & (9) & 779 & (69) & 347 & (31) \\
\hline \multirow[t]{4}{*}{ Lymph nodes removed } & None & 1845 & (50) & & & & & & & 1020 & (55) & 825 & (45) \\
\hline & $<10$ lymph nodes & 722 & (20) & & & & & & & 408 & (57) & 314 & (43) \\
\hline & $\geq 10$ lymph nodes & 697 & (19) & & & & & & & 528 & (76) & 169 & (24) \\
\hline & Unknown number & 394 & (11) & & & & & & & 261 & (66) & 133 & (34) \\
\hline \multirow[t]{5}{*}{ FIGO stage } & IA & 902 & (49) & 347 & (19) & 367 & (20) & 220 & (12) & 1525 & (83) & 311 & (17) \\
\hline & IB & 78 & (56) & 23 & (16) & 27 & (19) & 13 & (9) & 94 & (67) & 47 & (33) \\
\hline & IC & 737 & (54) & 252 & (18) & 240 & (18) & 131 & (10) & 541 & (40) & 819 & (60) \\
\hline & ПІА & 128 & (62) & 34 & (16) & 27 & (13) & 17 & (8) & 48 & (23) & 158 & (77) \\
\hline & IIIA1 & 0 & (0) & 66 & (58) & 36 & (31) & 13 & (11) & 9 & (8) & 106 & (92) \\
\hline \multirow[t]{3}{*}{ Hospital type } & General & 791 & (56) & 243 & (17) & 202 & (15) & 168 & (12) & 821 & (58) & 583 & (42) \\
\hline & Semi-specialized & 695 & (51) & 295 & (21) & 205 & (15) & 175 & (13) & 835 & (61) & 535 & (39) \\
\hline & Specialized & 359 & (40) & 184 & (21) & 290 & (33) & 51 & (6) & 561 & (63) & 323 & (37) \\
\hline Lymph node metastases & & & & 66 & (57) & 36 & (31) & 13 & (11) & 9 & (8) & 106 & (92) \\
\hline
\end{tabular}

\section{Lymph node dissection}

Lymph nodes were removed from 1813 patients (49.6\%). Less than ten lymph nodes were removed from 722 patients (39.8\%) and ten or more from 697 patients (38.4\%). The number removed from 394 patients (21.7\%) was unknown. Of those who underwent lymph node dissection, 115 (6.3\%) had lymph node metastases. The incidence of lymph node metastases in patients who had less than ten lymph nodes removed and ten or more lymph nodes removed, and in whom the amount of the removed lymph nodes was unknown, was $9.1 \%, 5.2 \%$ and 3.3\%, respectively. In patients with less than ten lymph 
nodes removed, more often only pathological lymph nodes were resected (55\%)(Figure 1).

Figure 1. Ratio of lymph nodes resected and lymph node metastases.

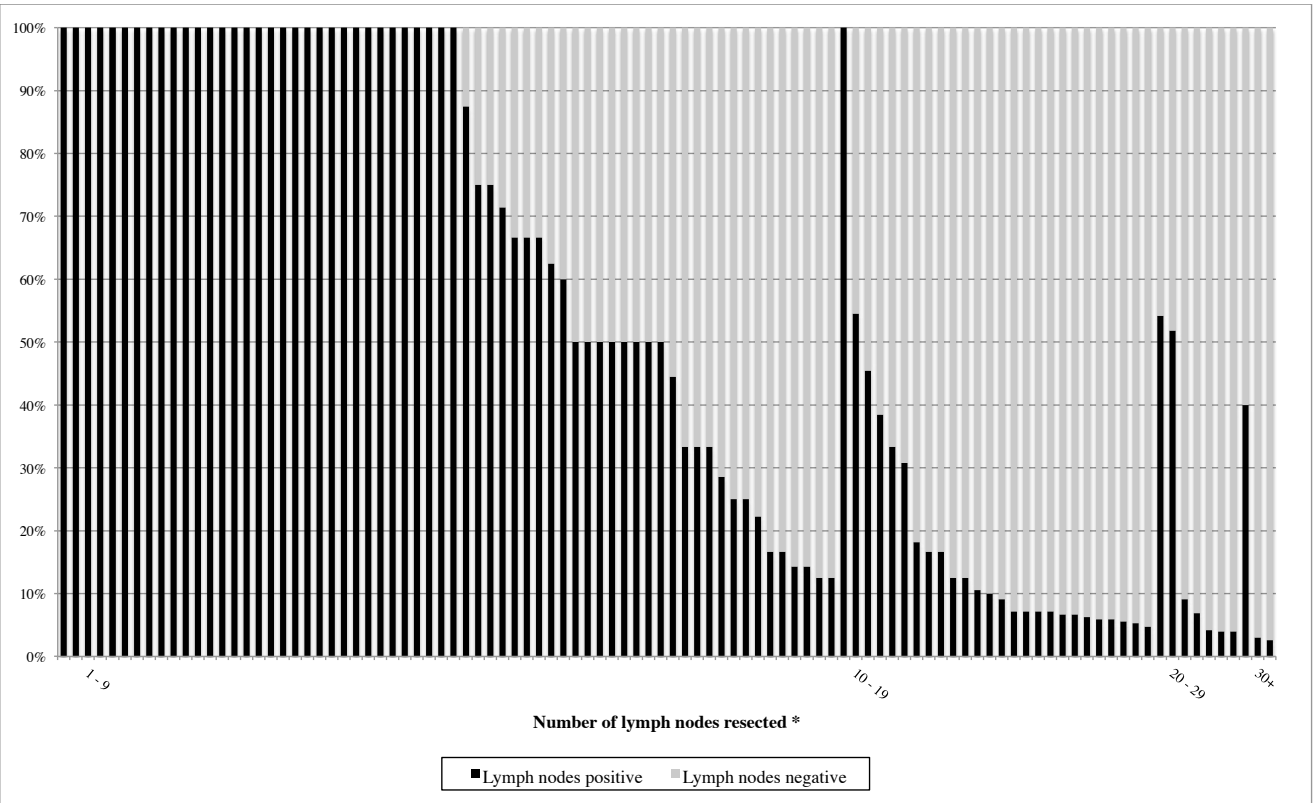

* 1-9 lymph nodes: in 66 out of 722 patients lymph node metastases

10-19 lymph nodes: in 25 out of 453 patients lymph node metastases

20-20 lymph nodes: in 8 out of 180 patients lymph node metastases $\geq 30$ lymph nodes: in 3 out of 64 patients lymph node metastases

The percentage of patients from whom lymph nodes were removed increased during the study period (Figure 2). Patients in specialized hospitals were more likely to undergo lymph node dissection than those in semi-specialized or general hospitals $(59.4 \% \mathrm{vs}$. $49.3 \%$ and $43.7 \%$ respectively; $\mathrm{p}<0.001$ ). This was mainly related to lymph node dissections in which ten or more lymph nodes were removed (32.8\% vs. $15.0 \%$ and $14.4 \% ; \mathrm{p}<0.001)$. 
Figure 2. Incidence of lymph node resection as part of the surgical staging procedure in patients with clinical early-stage EOC between 2000 and 2012.

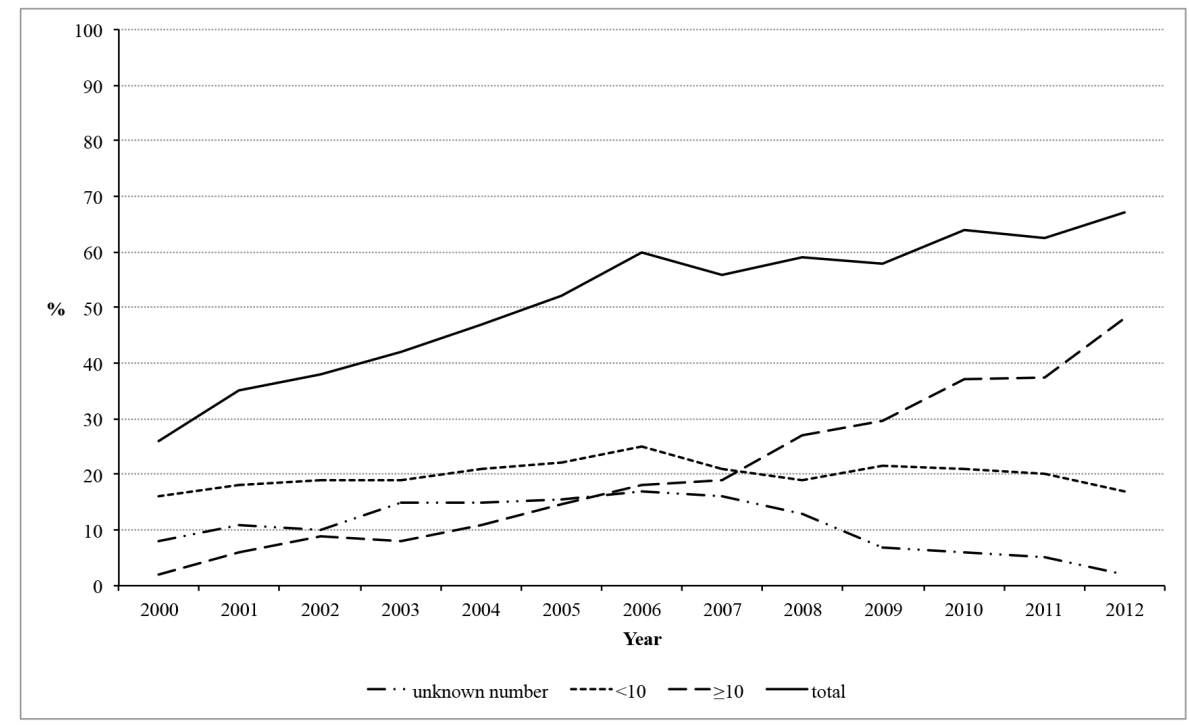

\section{Chemotherapy}

Adjuvant chemotherapy was administered to 1441 patients (39.4\%). Patients who underwent lymph node dissection were less likely to receive adjuvant chemotherapy than patients who did not. Of those that underwent dissection of ten or more lymph nodes, $24.3 \%$ received adjuvant chemotherapy compared with $43.5 \%$ of those with less than ten lymph nodes removed and $44.7 \%$ of those with no lymph nodes removed $(\mathrm{p}<0.001)$.

\section{Survival}

Adjuvant chemotherapy resulted in improved relative survival of patients from whom no lymph nodes were removed (RER 0.60; 95\% confidence interval (CI) 0.44-0.80).

Patients from whom lymph nodes were removed (including patients with lymph node metastases) had significantly better 5-year relative survival than patients that did not undergo lymph node dissection (89\% vs. 82\% respectively; RER 0.63; 95\% CI 0.52-0.77). This remained true after correcting for age, grade, subtype histology and FIGO stage (RER 0.64; 95\% CI 0.52-0.78)(Table 2). 
Table 2. Five-years survival RER estimators for patients without, compared to patients with removal of lymph nodes as part of the surgical staging procedure (univariable and multivariable risk).

\begin{tabular}{lll}
\hline univariable & multivariable \\
RER 95\% CI & RER 95\% CI \\
\hline
\end{tabular}

Lymph nodes

removed

$\begin{array}{lllll}\text { no } & 1.00 & \text { (reference) } & 1.00 & \text { (reference) } \\ \text { yes } & 0.63 & 0.52-0.77 & 0.64 & 0.52-0.78\end{array}$

Age $\quad 1.02 \quad 1.01-1.03 \quad 1.02 \quad 1.01-1.02$

Tumour grade

grade 11.00 (reference) 1.00 (reference)

$\begin{array}{llllll}\text { grade } 2 & 2.03 & 1.46-2.83 & 1.90 & 1.37-2.63\end{array}$

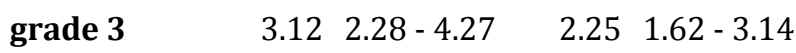

grade

$\begin{array}{lllll}\text { unknown } & 1.62 & 1.17-2.25 & 1.40 & 1.01-1.94\end{array}$

Tumour histology

serous $\quad 1.00$ (reference) 1.00 (reference)

mucinous $\quad \begin{array}{lllll}0.63 & 0.49-0.81 & 1.18 & 0.90-1.56\end{array}$

endometrioid $\quad 0.45 \quad 0.32-0.62 \quad 0.55 \quad 0.41-0.76$

$\begin{array}{lllll}\text { clear cell } & 1.05 & 0.79-1.38 & 1.23 & 0.92-1.65\end{array}$

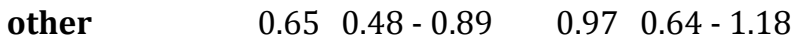

FIGO stage

\begin{tabular}{lllll} 
IA & 1.00 & (reference) & 1.00 & (reference) \\
IB & 1.82 & $1.16-2.88$ & 1.65 & $1.05-2.61$ \\
IC & 1.72 & $1.39-2.14$ & 1.56 & $1.25-1.94$ \\
IIA & 2.83 & $2.02-3.96$ & 2.63 & $1.68-3.32$ \\
IIIA1 & 3.61 & $2.31-5.62$ & 2.61 & $1.66-4.11$ \\
\hline
\end{tabular}


There was a positive correlation between the number of removed lymph nodes and overall survival (after excluding patients with lymph node metastases) (Figure 3). There was no difference in survival between the groups in whom $\geq 20-30$ and $\geq 30$ lymph nodes were removed.

Figure 3. Overall survival of patients with FIGO stage I-IIA EOC related to the number of dissected lymph nodes.

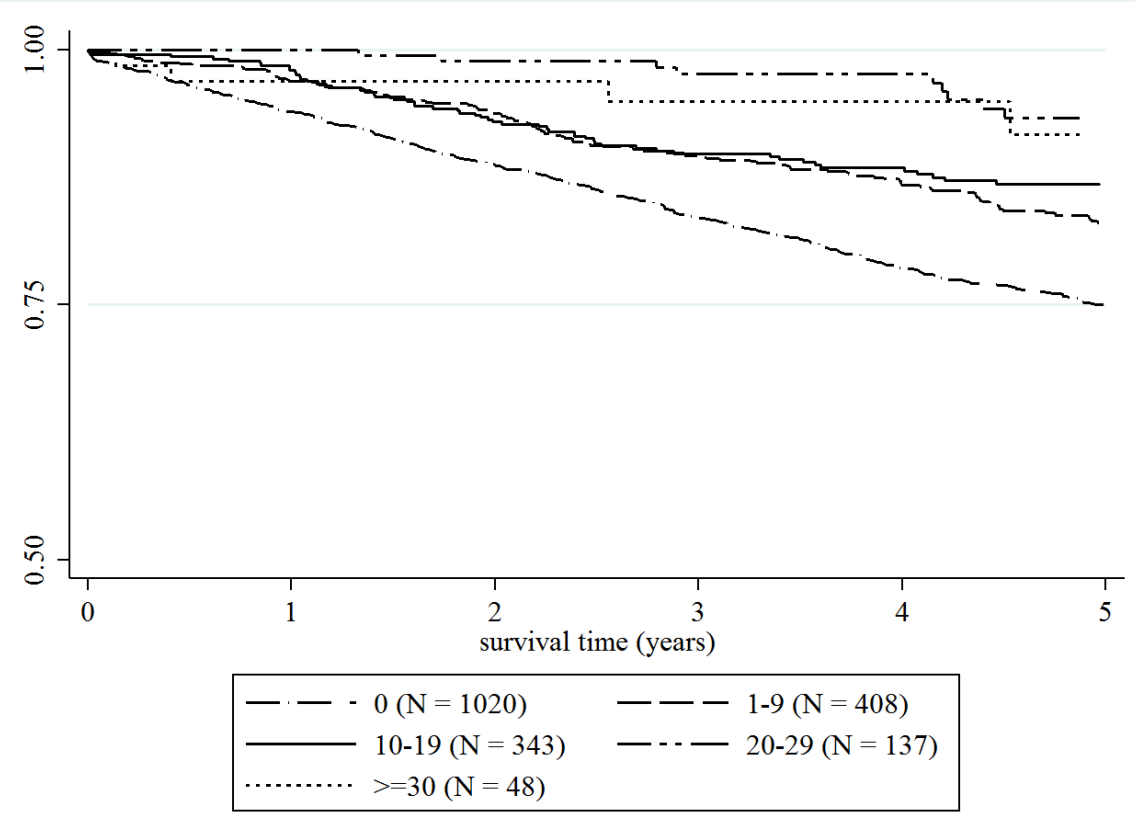


The 5-year relative survival for patients with lymph node metastases was 78\% (CI 6886\%)(Figure 4).

Figure 4. Overall survival of patients with FIGO IIIA1 EOC.

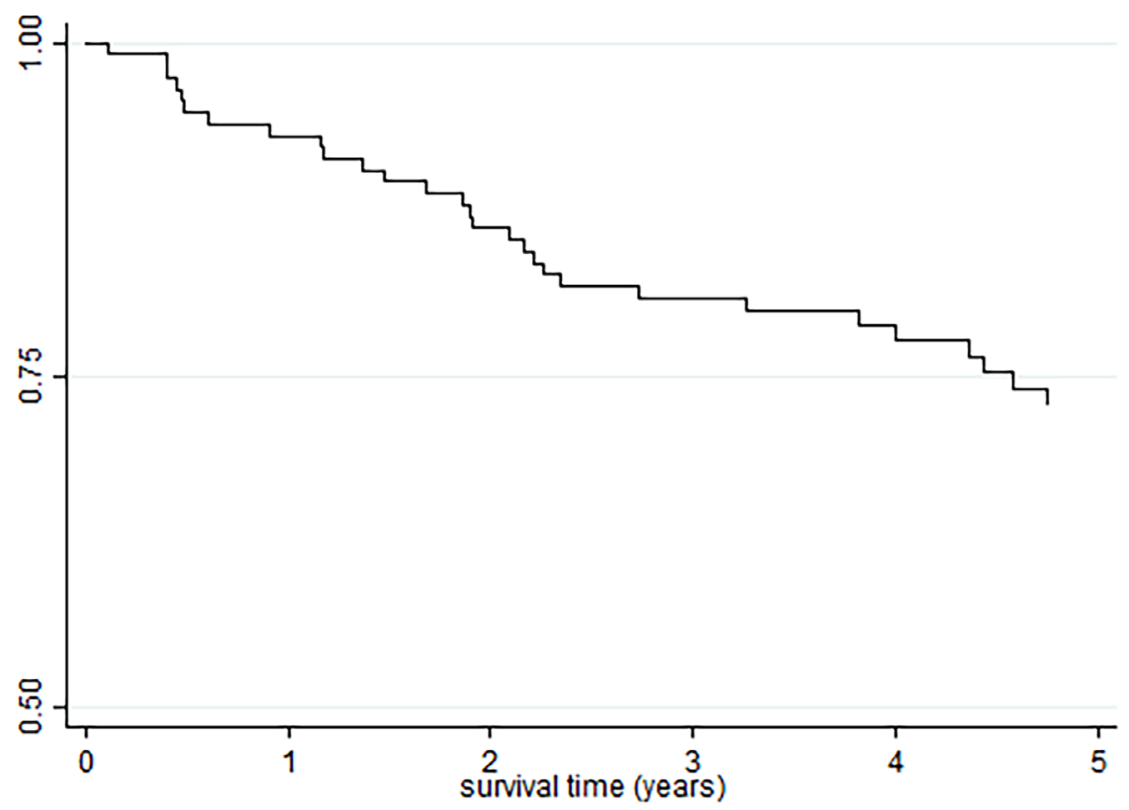


The relative survival of patients that underwent dissection of ten or more lymph nodes without adjuvant chemotherapy was significantly better than that of patients with no lymph node dissection but with adjuvant chemotherapy (RER 0.46; 95\% CI 0.34-0.63) (Figure 5).

Figure 5. Overall survival of FIGO stage I-IIA patients without lymph node sampling and adjuvant chemotherapy, and patients with lymph node dissection of ten lymph nodes or more without adjuvant chemotherapy.

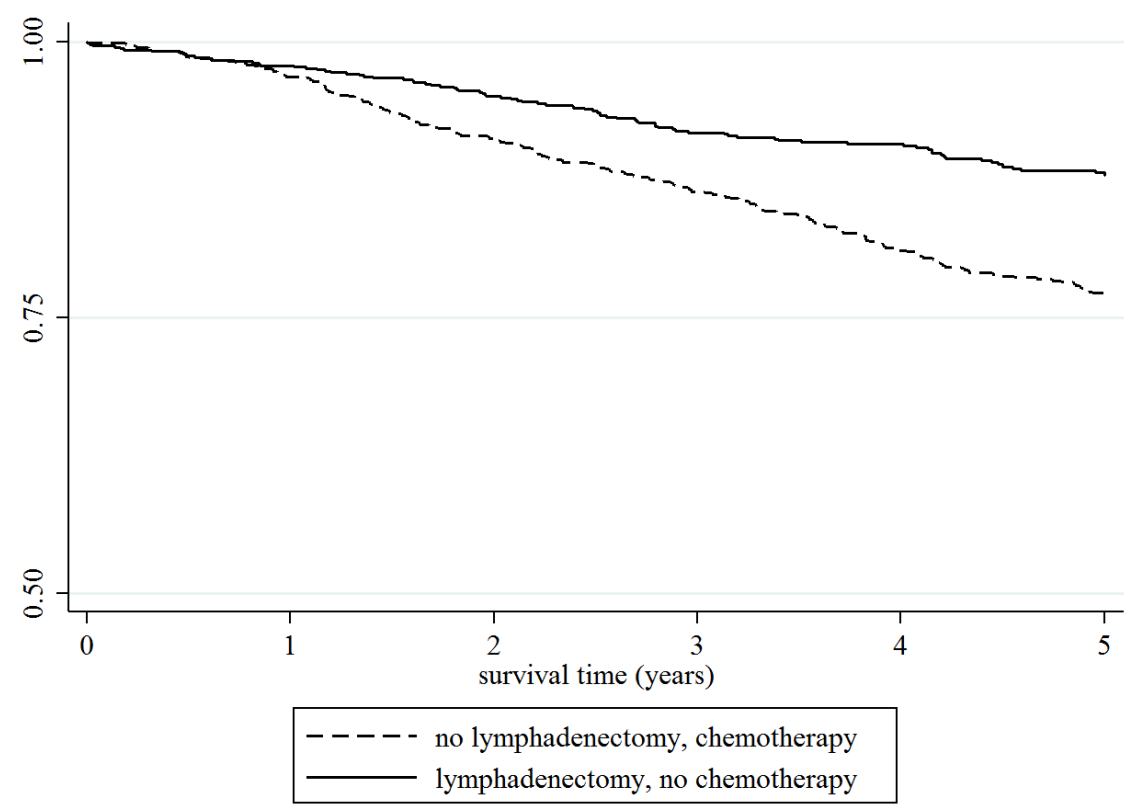

This was also true after correcting for age, grade, subtype histology and FIGO stage (RER 0.60; 95\% CI 0.41-0.87)(Table 3).

Table 3. Five-years survival RER estimators for patients without removal of lymph nodes and adjuvant chemotherapy compared with patients with removal of lymph nodes and no adjuvant chemotherapy (univariable and multivariable risk). 


\begin{tabular}{|c|c|c|c|c|}
\hline & \multicolumn{2}{|c|}{ univariable } & \multicolumn{2}{|c|}{ multivariable } \\
\hline & $\overline{\text { RER }}$ & $95 \% \mathrm{CI}$ & RER & $95 \% \mathrm{CI}$ \\
\hline \multicolumn{5}{|l|}{ Lymph nodes / } \\
\hline \multicolumn{5}{|l|}{ chemotherapy } \\
\hline $\begin{array}{l}\text { no lymph nodes, } \\
\text { with chemotherapy }\end{array}$ & 1.00 & (reference) & 1.00 & (reference) \\
\hline $\begin{array}{l}\geq 10 \text { lymph nodes, } \\
\text { no chemotherapy }\end{array}$ & 0.46 & $0.34-0.63$ & 0.60 & $0.41-0.88$ \\
\hline Age * & 1.00 & $0.99-1.01$ & 1.00 & $0.98-1.01$ \\
\hline \multicolumn{5}{|l|}{ Tumour grade } \\
\hline grade 1 & 1.00 & (reference) & 1.00 & (reference) \\
\hline grade 2 & 1.77 & $1.08-2.89$ & 1.86 & $1.13-3.07$ \\
\hline grade 3 & 2.35 & $1.46-3.80$ & 2.05 & $1.22-3.45$ \\
\hline grade unknown & 1.98 & $1.22-3.19$ & 1.68 & $1.00-2.84$ \\
\hline \multicolumn{5}{|l|}{ Tumour histology } \\
\hline serous & 1.00 & (reference) & 1.00 & (reference) \\
\hline mucinous & 0.80 & $0.53-1.20$ & 0.98 & $.063-1.51$ \\
\hline endometrioid & 0.42 & $0.26-0.68$ & 0.44 & $0.27-0.73$ \\
\hline clear cell & 1.07 & $0.72-1.58$ & 1.09 & $0.71-1.68$ \\
\hline other & 1.14 & $0.74-1.75$ & 1.11 & $0.72-1.72$ \\
\hline \multicolumn{5}{|l|}{ FIGO stage } \\
\hline IA & 1.00 & (reference) & 1.00 & (reference) \\
\hline IB & 1.00 & $0.43-2.30$ & 0.91 & $0.39-2.12$ \\
\hline IC & 1.25 & $0.89-1.76$ & 1.30 & $0.92-1.83$ \\
\hline IIA & 1.72 & $1.03-2.90$ & 1.83 & $1.08-3.09$ \\
\hline
\end{tabular}

* continuous variable. 
In the cases of patients with FIGO I-IIA EOC that underwent dissection of ten or more lymph nodes, we found no difference in relative survival between those that received chemotherapy $(n=527)$ and those that did not $(n=134)$ (RER 0.51; 95\% CI 0.15-1.64) (Figure 6).

Figure 6. Overall survival of patients with FIGO I-IIA EOC that underwent a lymph node dissection of ten or more lymph nodes with and without adjuvant chemotherapy.

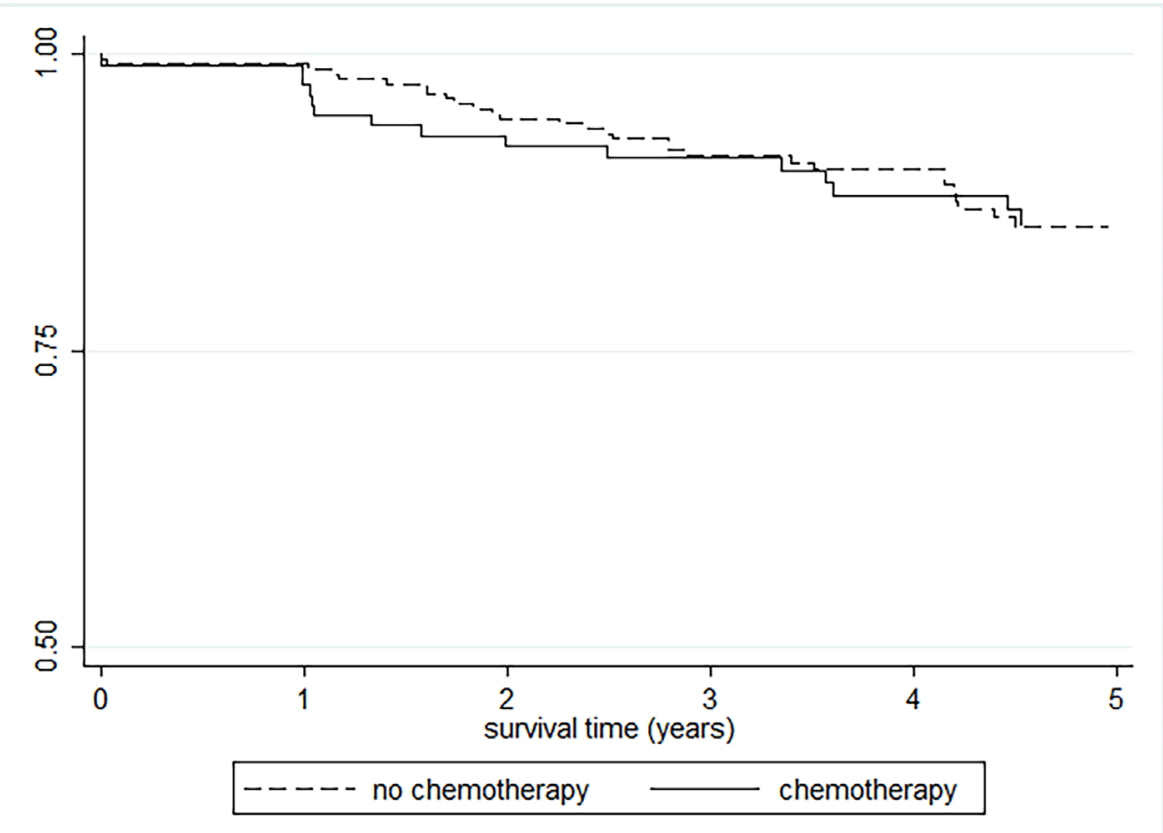

Examination of a subgroup of patients with high-risk features (FIGO IC and IIA, and/or differentiation grade 3 and/or clear cell histology) that underwent dissection of ten or more lymph nodes revealed that adjuvant chemotherapy did not result in a significant improvement in relative survival (RER 0.90; 95\% CI 0.46-1.99, $\mathrm{n}=244$ with and $\mathrm{n}=123$ without adjuvant chemotherapy). (Figure 7). 
Figure 7. Overall survival of patients with high-risk FIGO I-IIA EOC that underwent a lymph node dissection of ten or more lymph nodes with and without adjuvant chemotherapy.

* FIGO Ic and IIA and/or differentiation grade 3 and/or clear cell histology.

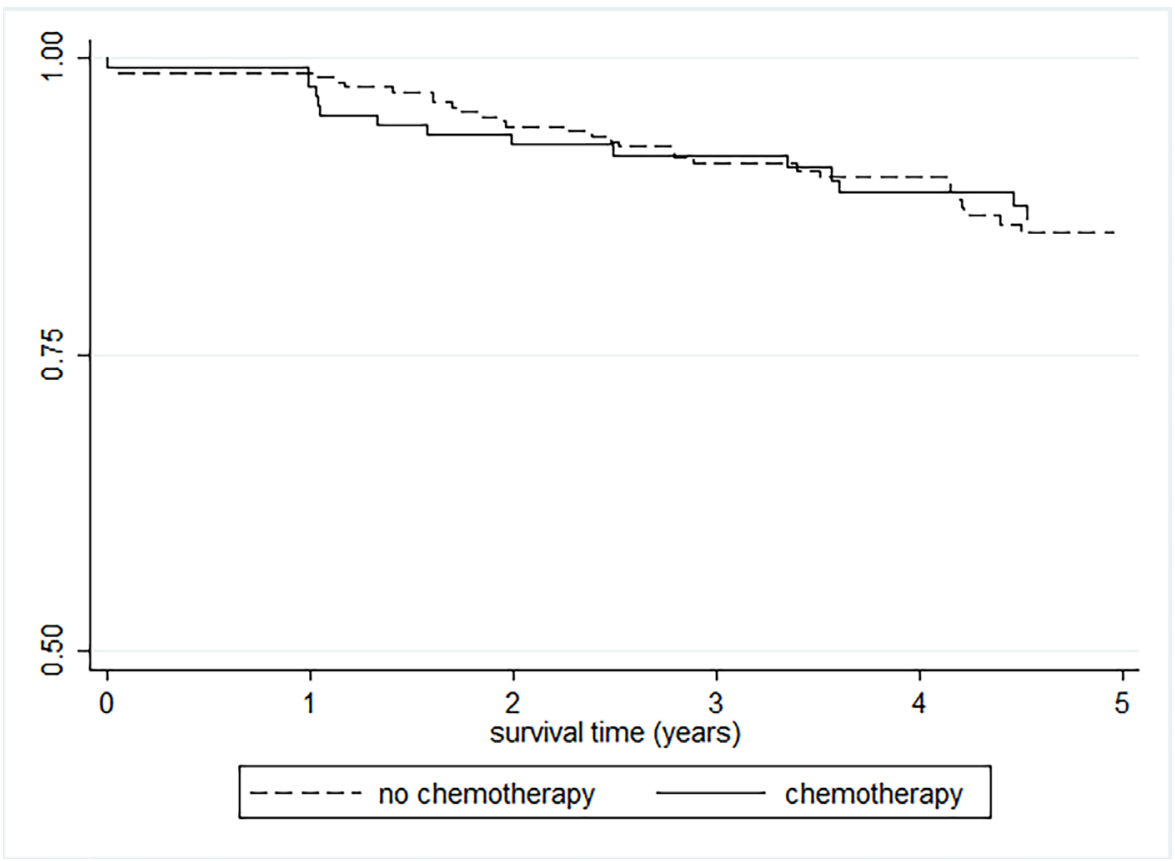

\section{Discussion}

To the best of our knowledge, this is the largest cohort study of clinical early-stage EOC that examines the influence of lymph node dissection and chemotherapy on survival. We found that clinical early-stage EOC patients that underwent lymph node dissection as part of a surgical staging procedure had better relative survival than patients that did not, even when patients with lymph node metastases were included. For those from whom ten or more lymph nodes were removed, adjuvant chemotherapy did not improve relative survival.

In early-stage EOC, FIGO recommends a complete para-aortic and pelvic lymphadenectomy. ${ }^{4}$ In the present cohort study, only $50 \%$ of patients underwent lymph node dissection, although this improved from $26 \%$ in 2000 to $67 \%$ in 2012. Moreover, the percentage of patients from whom ten or more nodes were removed also increased during 
the study period (from $2.3 \%$ to $47.6 \%$ ). These figures are consistent with the reported incidence of lymph node staging worldwide, which varies between $10 \%$ and $30 \% .13$ There are several explanations for why clinicians do not comply with FIGO recommendations: they might not be able to resect lymph nodes, or they might decide to give adjuvant chemotherapy irrespective of lymph node status. Also, patients might reject adjuvant chemotherapy, or they might not be in a condition to receive chemotherapy. ${ }^{14}$ Our finding of an increasing number of lymph node dissections over time suggests that improved knowledge of the technique (and the importance) of lymph node dissection would lead to better compliance with guidelines and to improved survival.

Overall, metastases were found in $6.3 \%$ of patients that underwent lymph node dissection. This percentage is low when compared with that reported in other studies. A review on lymph node metastases in clinical early-stage EOC suggests that the incidence varies from $6.1 \%$ to $29.6 \%$ (mean $14.2 \%$ ). ${ }^{15}$ Surprisingly, we found that lymph node metastases were more common in patients from whom less than ten lymph nodes were removed than in those from whom ten or more were removed (9.1\% vs. 5.2\% respectively). The probable explanation for this is that, in a number of patients, lymph node dissection was halted when metastasis was detected. This fits with the observation that of patients from whom less than 10 lymph nodes were removed, in a high percentage of cases (55\%) all lymph nodes were pathological. Therefore, not all patients from whom fewer than ten lymph nodes were removed can be considered incompletely staged.

The five year overall survival for FIGO stage IIIA1 patients was 78\%. This superior survival when compared to other advanced stage patients, is in accordance with previous reports. ${ }^{16-17}$

The minimum number of lymph nodes required for adequate lymph node staging remains controversial. Here, we found that overall survival improved in line with the number of lymph nodes removed. This correlation was also observed by others, and is probably based on stage migration, i.e., patients with (occult) lymph node metastases progressing to advanced stage disease.9, 18-20 There was no difference in survival between those from whom 20-29, and those from whom $\geq 30$ lymph nodes were removed. Above a certain number of lymph nodes, the identification of lymph node metastases is probably less 
likely. Therefore, adequate dissection of at least 10 , but preferably 20 or more lymph nodes should be standard procedure for the staging of early-stage EOC.

We found that adjuvant chemotherapy significantly improved the relative survival of patients that did not undergo lymph node staging. This is in agreement with a Cochrane review meta-analysis on adjuvant chemotherapy. ${ }^{21}$ This review included a subgroup analysis of patients that underwent a complete staging procedure plus or minus chemotherapy; the results showed no difference in overall five year survival (hazard ratio 1.22; 95\% CI 0.63-2.37). However, the subgroup analysis was based on only 117 patients in each group, mainly because a significant number of patients were incompletely staged. ${ }^{22}$ Similarly, a retrospective study of 721 patients showed that adjuvant chemotherapy for completely staged patients did not improve survival. ${ }^{19}$

In the present study, we found that patients from whom ten or more lymph nodes were removed, but did not undergo adjuvant chemotherapy, had significantly better relative survival than patients without lymph node removal that did undergo adjuvant chemotherapy. This remained true after correcting for age, differentiation grade, subtype histology and stage. This fits with the analysis of the mature data of the ACTION trial. ${ }^{22}$ These results could be construed as evidence supporting a therapeutic role for lymph node dissection. However, because we excluded patients with lymph node metastases from the group of patients that underwent lymph node dissection, the survival benefit may be explained by stage migration.

It is suggested that adjuvant chemotherapy after an adequate staging procedure should be considered for a selective group of high-risk patients. ${ }^{23}$ The latter assumption is based on an analysis of high-risk patients in a retrospective study, as well as on extrapolation from experience in patients with advanced stage EOC. In the ACTION trial, a subgroup analysis was done of high-risk patients (defined as grade 2-3 stages IA-IB, all stages IC and IIA, and all patients with clear cell cancer), in which the completeness of staging was taken into account. ${ }^{22}$ In completely staged patients no difference in survival was observed between patients with $(n=76)$ and without $(n=75)$ adjuvant chemotherapy. We also found that high-risk patients (defined as tumour grade 3 and/or FIGO stage IC and IIA and/or clear cell histology) from whom ten or more lymph nodes were removed as part of a staging 
procedure did not benefit from adjuvant chemotherapy (241 with and 116 without adjuvant chemotherapy).

To analyse treatment differences between hospitals, we examined the frequency of lymph node dissection in different types of hospital. Lymph node dissection was performed less often in general hospitals than in specialized hospitals. These data are concordant with those from other studies and possibly is related to a lack of surgical skills. ${ }^{8,14,23}$

The strength of this study is the large number of patients examined ( $\mathrm{n}=3658)$ and the fact that it is based on a nationwide analysis. A nationwide analysis precludes the influence of different treatment habits in different regions, even within one country. ${ }^{24}$ The major limitation is the retrospective design. First, we had no information about patient characteristics such as comorbidities, which could have influenced decisionmaking regarding the extent of the lymph node dissection or about whether to undertake adjuvant chemotherapy. Second, data about the number of removed lymph nodes was missing for 394 patients. Also the locations of the lymph nodes removed was not known. Third, no information was available about other aspects of the staging procedure, such as omentectomy, peritoneal biopsies and quality of inspection for residual tumour. For apparent early-stage EOC, random peritoneal biopsies and omentectomy are of little diagnostic value beyond careful inspection. ${ }^{25-27}$ However taking these biopsies is a good guarantee that inspection and palpation has actually been carried out. In patients in whom lymph nodes were resected, possibly a more thorough inspection of the abdominal cavity was performed. Fourth, we did not perform a centralized pathology review. This could also have influenced our results. ${ }^{28}$

\section{Conclusions}

This nationwide cohort study shows that removal of lymph nodes as part of a staging procedure for early-stage EOC contributes to improved relative survival. The more lymph nodes dissected, the better the overall survival. Adjuvant chemotherapy in completely staged patients does not to improve relative survival, not even in high-risk patients. 


\section{References}

1. www.seer.cancer.gov

2. Benedet JL, Bender H, Jones H $3^{\text {rd }}$, Ngan HY, Pecorelli S. FIGO staging classifications and clinical practice guidelines in the management of gynecologic cancers. FIGO Committee on Gynecologic Oncology. Int J Gynaecol Obstet 2000;70:209-262.

3. Collinson F, Qian W, Fossati R, Lissoni A, Williams C, Parmar M, Ledermann J, Colombo N, Swart A, ICON1 collaborators. Optimal treatment of early-stage ovarian cancer. Ann Oncol 2014;25:1165-1171.

4. Prat J. FIGO Committee on Gynecologic Oncology: Staging classification for cancer of the ovary, fallopian tube, and peritoneum. Int J Gynecol Obstet 2014;124:1-5.

5. Di Re F, Baiochocci G. Value of lymph node assessment in ovarian cancer: status of the art at the end of the second millennium. Int J Gynecol Cancer 2000;10:435-442.

6. Colombo N, Guthrie D, Chiari S Parmar M, Qian W, Swart AM, Torri V, Williams C, Lissoni A, Bonazzi C; International Collaborative Ovarian Neoplasm (ICON) collaborators. International collaborative ovarian neoplasm trial 1: a randomised trial of adjuvant chemotherapy in women with early-stage ovarian cancer. J Natl Cancer Inst 2003;95:125-132.

7. Trimbos JB, Vergote I, Bolis G, Vermorken JB, Mangioni C, Madronal C, Franchi M, Tateo S, Zanetta G, Scarfone G, Giurgea L, Timmers P, Coens C, Pecorelli S; EORTCACTION collaborators. Impact of adjuvant chemotherapy and surgical staging in early-stage ovarian carcinoma: European Organization for Research and Treatment of Cancer- Adjuvant chemotherapy in ovarian neoplasm trial. J Natl Cancer Inst 2003;95:113-124.

8. Trimbos JB, Parmar M, Vergote I, Guthrie D, Bolis G, Colombo N, Vermorken JB, Torri V, Mangioni C, Pecorelli S, Lissoni A, Swart AM. International collaborative ovarian neoplasm trial 1 and adjuvant chemotherapy in ovarian neoplasm trial: two parallel randomized phase III trials of adjuvant chemotherapy in patients with early-stage ovarian carcinoma. J Natl Cancer Inst 2003;95:105-112.

9. Carnino F, Fuda G, Ciccone G, Iskra L, Guercio E, Dadone D, Conte PF. Significance of lymph node sampling in epithelial carcinoma of the ovary. Gynecol Oncol 1997;65:467-472.

10. Schouten LJ, van den Brandt PA, Jager JJ. Completeness of cancer registration in Limburg, The Netherlands. Int J Epidemiol 1993;22:369-376. 
11. Schouten L, Jager J, Van den Brandt P. Quality of cancer registry data: a comparison of data provided by clinicians with those of registration personnel. Brit J Cancer 1993;68:974.

12. Ederer F, Axtell LM, Cutler SJ. The relative survival rate: a statistical methodology. Natl Cancer Inst Monogr 1961;6:101-121.

13. Tropé C, Kaern J. Adjuvant chemotherapy for early-stage ovarian cancer: review of the literature. J Clin Oncol 2007;25:2909-2920.

14. Timmers PJ, Zwinderman AH, Coens C, Vergote I, Trimbos JB. Understanding the problem of inadequately staging early ovarian cancer. Eur J Cancer 2010;46:880884.

15. Kleppe M, Wang T, Van Gorp T, Slangen BFM, Kruse AJ, Kruitwagen RFPM. Lymph node metastasis in stage I and II ovarian cancer: a review. Gynecol Oncol 2011;123:610-614.

16. Onda T, Yoshikawa H, Yasugi T, Mishima M, Nakagawa S, Yamada M, Matsumoto K, Taketani Y. Patients with ovarian carcinoma upstaged to stage III after systematic lymphadenectomy have similar survival to Stage I/II patients and superior survival to other Stage III patients. Cancer 1998;83:1555-1560.

17. Baek SJ, Park JY, Kim DY, Kim JH, Kim YM, Kim YT, Nam JH. Stage IIIC epithelial ovarian cancer classified solely by lymph node metastasis has a more favorable prognosis than other types of stage IIIC epithelial ovarian cancer. JGO 2008;19:223228.

18. Maggioni A, Benedetti Panici P, Dell'Anna T, Landoni F, Lissoni A, Pellegrino A, Rossi RS, Chiari S, Campagnutta E, Greggi S, Angiolo R, Manci N, Calcagno M, Scambia G, Fossati R, Floriani I, Torri, Grassi R, Mangioni C. Randomised study of systematic lymphadenectomy in patients with epithelial ovarian cancer macroscopically confined to the pelvis. Br J Cancer 2006;18:699-704.

19. Cress RD, Bauer K, O’Malley CD Kahn AR, Schymura MJ, Stewart SL, Leiserowitz GS. Surgical staging of early-stage epithelial ovarian cancer: results from the CDC-NPCR ovarian patterns of care study. Gynecol Oncol 2011;121:94-99.

20. Chan JK, Munro EG, Cheung MK, Husain A, Teng NN, Berek JS, Osann K. Association of lymphadenectomy and survival in stage I ovarian cancer patients. Obstet Gynecol 2007;109:12-19. 
21. Winter-Roach BA, Kitchener HC, Lawrie TA. Adjuvant (post-surgery) chemotherapy for early stage epithelial ovarian cancer. Cochrane Database Syst 2012;Rev 3:CD004706.

22. Trimbos B, Timmers P, Pecorelli S, Coens C, Ven K, van der Burg M, Casado A. Surgical staging and treatment of early ovarian cancer: long-term analysis from a randomized trial. J Natl Cancer Inst 2010:102;982-987.

23. Vergote I, De Brabanter J, Fyles A, Bertelsen K, Einhorn N, Sevelda P, Gore ME, Kaern J, Verreist H, Sjövall K, Timmerman D, Vandewalle J, Van Gramberen M, Tropé CG. Prognostic importance of degree of differentiation and cyst rupture in stage I invasive epithelial ovarian carcinoma. Lancet 2001;357:176-182.

24. Ulanday KT, Ward KK, Macera CA, Ji M, Plaxe SC. Regional variation in surgical assessment of lymph node for staging among women with early-stage epithelial ovarian cancer. Gynecol Oncol 2014;132:411-415.

25. Powless CA, Bakum-Gamez JN, Aletti G, Cilby WA. Random peritoneal biopsies have limited value in staging of apparent early-stage epithelial ovarian cancer after thorough exploration. Gynecol Oncol 2009;115:86-89.

26. Lee JY, Kim HS, Chung HH, Kim JW, Park NH, Song YS. The role of omentectomy and random peritoneal biopsies as part of comprehensive surgical staging in apparent early-stage epithelial ovarian cancer. Ann Surg Oncol 2014;21:2762-2766.

27. McNally L, Teng NNH, Kapp DS, Karam A. Does omentecomy in epithelial ovarian cancer affect survival? An analysis of the surveillance, epidemiology and end results database. Int J Gynecol Cancer 2015;25:607-615.

28. Verleye L, Ottevanger PB, Kristensen GB, Ehlen T, Johnson N, van der Burg ME, Reed NS, Verheijen RH, Gaarenstroom KN, Mosgaard B, Seoane JM, van der Velden J, Lotocki R, van der Graaf W, Penninckx B, Coens C, Stuart G, Vergote I. Quality of pathology reports for advanced ovarian cancer: are we missing essential information? An audit of 479 pathology reports from the EORTC-GCG 55971/NCICCTG OV13 neoadjuvant trial. Eur J Cancer 2011;47:57-64. 



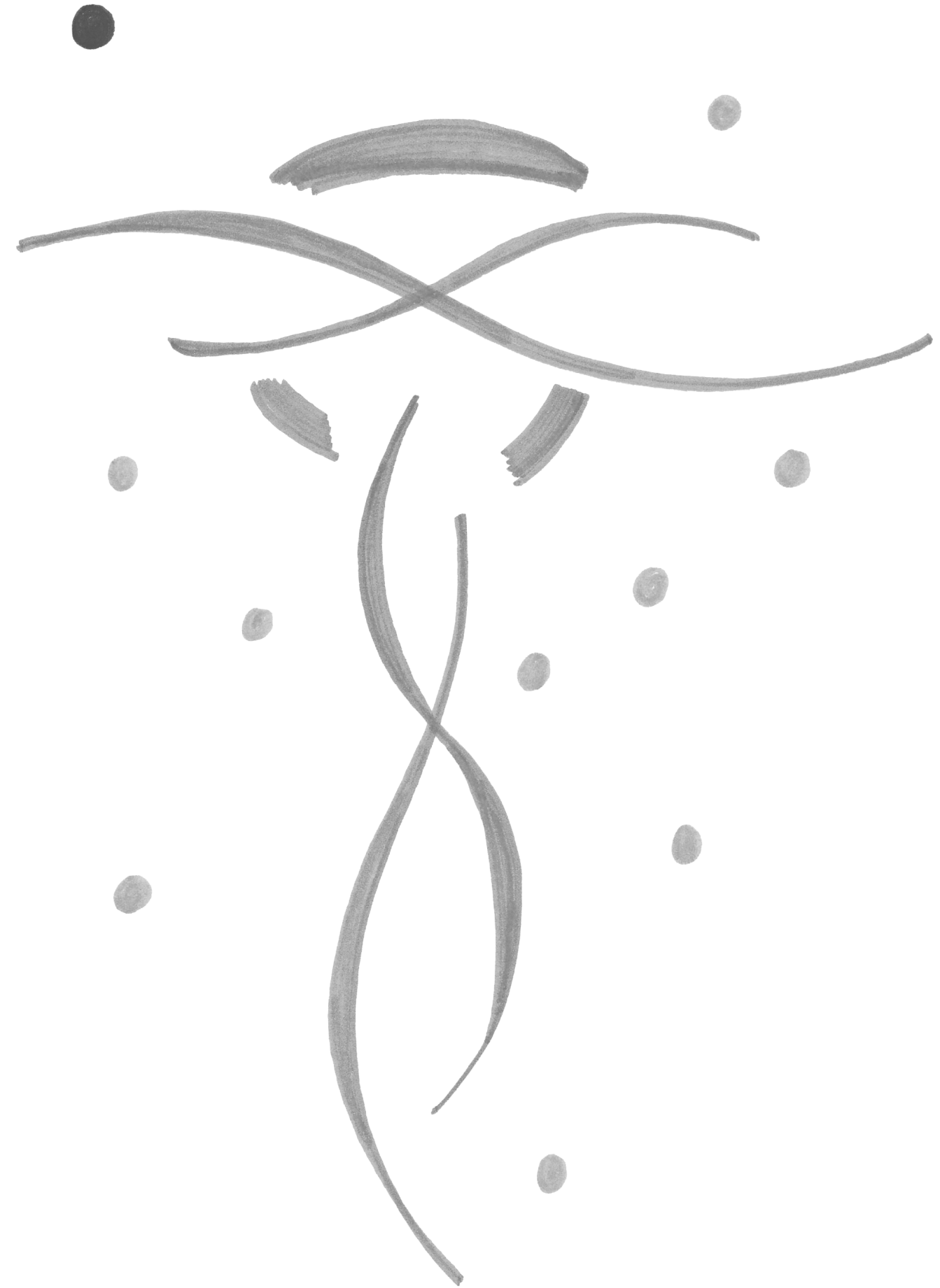




\section{Chapter 8}

\section{Valorisation addendum}




\section{Why is this thesis relevant?}

Although clinical early-stage ovarian cancer and lymph node metastases have been studied for many years, still numerous questions both regarding the staging procedure as well as treatment are unsolved. In this thesis the incidence of lymph node metastasis in clinical early-stage ovarian cancer was reviewed, and in a large retrospective study the value of both the removal of lymph nodes as part of a surgical staging procedure and adjuvant chemotherapy was studied. Furthermore a new sentinel node procedure was tested in which the tracers were injected in the ovarian ligaments instead of in the ovary itself.

\section{Relevance}

Value of an adequate lymph node sampling as part of a surgical staging procedure A lymphadenectomy is a radical procedure that is associated with morbidity, including nerve and vessel injury, increased blood loss, increased operating time, and the formation of lymphocysts and lymphedema. According to the International Federation of Gynaecology and Obstetrics (FIGO), ovarian cancer with lymph node metastases is classified as FIGO stage IIIA disease for which adjuvant chemotherapy is indicated. Our review on the incidence of lymph node metastases in early-stage ovarian cancer has shown that the overall incidence of lymph node metastases is $14 \%$, which cannot be ignored. Only in case of a grade 1 mucinous ovarian tumour an adequate lymph node staging may be omitted.

Value of adjuvant chemotherapy in patients with early-stage invasive epithelial ovarian cancer

The role of adjuvant chemotherapy in adequately staged patients without lymph node metastases is still controversial. Some studies have indicated that, irrespective of the extensiveness of a staging procedure, adjuvant chemotherapy improves survival. Others have stated that adjuvant chemotherapy in these patients is only indicated in case of high risk factors such as FIGO stage IC and IIA and/or differentiation grade 3 and/or clear cell histology.

Our large retrospective study on the value of adjuvant chemotherapy has provided further evidence that, after an adequate staging procedure including at least 10 and preferably 20 
or more lymph nodes, adjuvant chemotherapy is not indicated, even in patients with high risk factors.

SN technique by injecting tracers into the ovarian ligaments

Research on sentinel node technique in ovarian cancer is scarce. Only two studies, performed in patients with endometrial cancer, SNs were identified after injecting the tracers in the ovarian cortex. Injecting tracer in a tumour, especially one in the ovary can cause tumour spillage, and thereby metastasis. By injecting the tracers in the ovarian ligaments we have introduced a new technique for the detection of the sentinel node in ovarian cancer which appears to be promising.

Knowledge of the lymph drainage patterns of the ovary is essential to know where to look for positive lymph nodes during surgery. In the regions where lymph node metastases can be found, up to 200 or more lymph nodes are found. To remove them all would be an extensive procedure, leading to more morbidity among others increased blood loss and higher risk of lymphocysts and lympedema.

Our study in which the lymphatic drainage pathways of the ovaries in three human foetal pelves immunohistochemically were studied provided further evidence that the ovarian ligaments are a good alternative site for injection of the tracers.

\section{Target groups}

The results of this thesis are interesting for gynaecologists, gynaecologic oncologists, medical oncologists, pathologists, nuclear medicine physicians, treatment developers and, most important, hopefully in the near future also for patients. When indeed the SN procedure in ovarian cancer appears to have a high accuracy as is the case for other tumours such as breast and vulvar cancer, more patients with clinical early-stage cancer will obtain an adequate staging procedure without the accompanying morbidity related to a complete pelvic and para-aortal lymphadenectomy.

\section{Activities and Innovation}

By injecting the tracers in the ovarian ligaments we have introduced a new technique for the detection of the sentinel node in ovarian cancer. In our feasibility study we found a 
high detection rate of sentinel nodes and no side effects were seen due to the procedure. Also the procedure appeared to be safe for the involved health care workers.

Our results have been published in scientific research journals. We have had the opportunity to discuss our findings nationally and internationally to gain more attention for this important topic. We still need to further explore this new technique and this leads to more research.

\section{Schedule and implementation}

The results of the feasibility study on identifying SNs in patients with ovarian cancer are promising, but needs additional research. Remaining questions which need to be answered are:

- Is a SN procedure through injection of the tracers in the ovarian ligaments still feasible immediately after a salpingo-oohorectomy is performed?

In our feasibility study in which tracers were injected into the ovarian ligaments to identify possible sentinel nodes, the injection occurred before the adnex was resected for frozen section. However, this means that in a significant number of patients the injection of tracers is unnecessary when the result of frozen section indicates a benign of borderline lesion for which no lymph node resection is necessary.

- Is a SN procedure through injection of the tracers in the ovarian ligaments still feasible in patients in whom the malignant ovarian tumour has already been resected? This concerns patients in whom previously the ovarian tumour has been resected because it was not suspected to be malignant.

- Is the fact that, in our study, the majority of the sentinel nodes identified by the gamma probe did not show blue colorization is caused by a long time-interval between injection and retroperitoneal exploration?

- Can the use of a mobile gamma camera during the surgical procedure enhance intraoperative detection of sentinel nodes?

- Can multimodality radioactive and fluorescence guidance enhances intraoperative detection of sentinel nodes?

Some of these questions will be answered in the SONAR 2 study that recently is initiated in the Maastricht University Medical Centre+, in cooperation with two other gynaecologic oncology centres (Radboud University Medical Centre and Antoni van Leeuwenhoek Hospital Amsterdam). 
When these questions are answered, hopefully resulting in a more profound technique, a (inter)national prospective multi-centre trial could be done to determine the diagnostic accuracy of the sentinel lymph node procedure in patients with ovarian cancer. In this study, after removal of the first sentinel lymph node, subsequently an adequate lymph node sampling (at least 10, and preferably 20 or more lymph nodes from the pelvic and para-aortal regions) is performed to establish the incidence of false negative lymph nodes and the incidence of unrecognized sentinel nodes. This gives MUMC+ the opportunity to become the leading centre for research concerning the sentinel node procedure in ovarian cancer.

Hopefully, when the technique is proven to be effective, it will become part of the daily practice in treatment of early-stage ovarian cancer worldwide. 


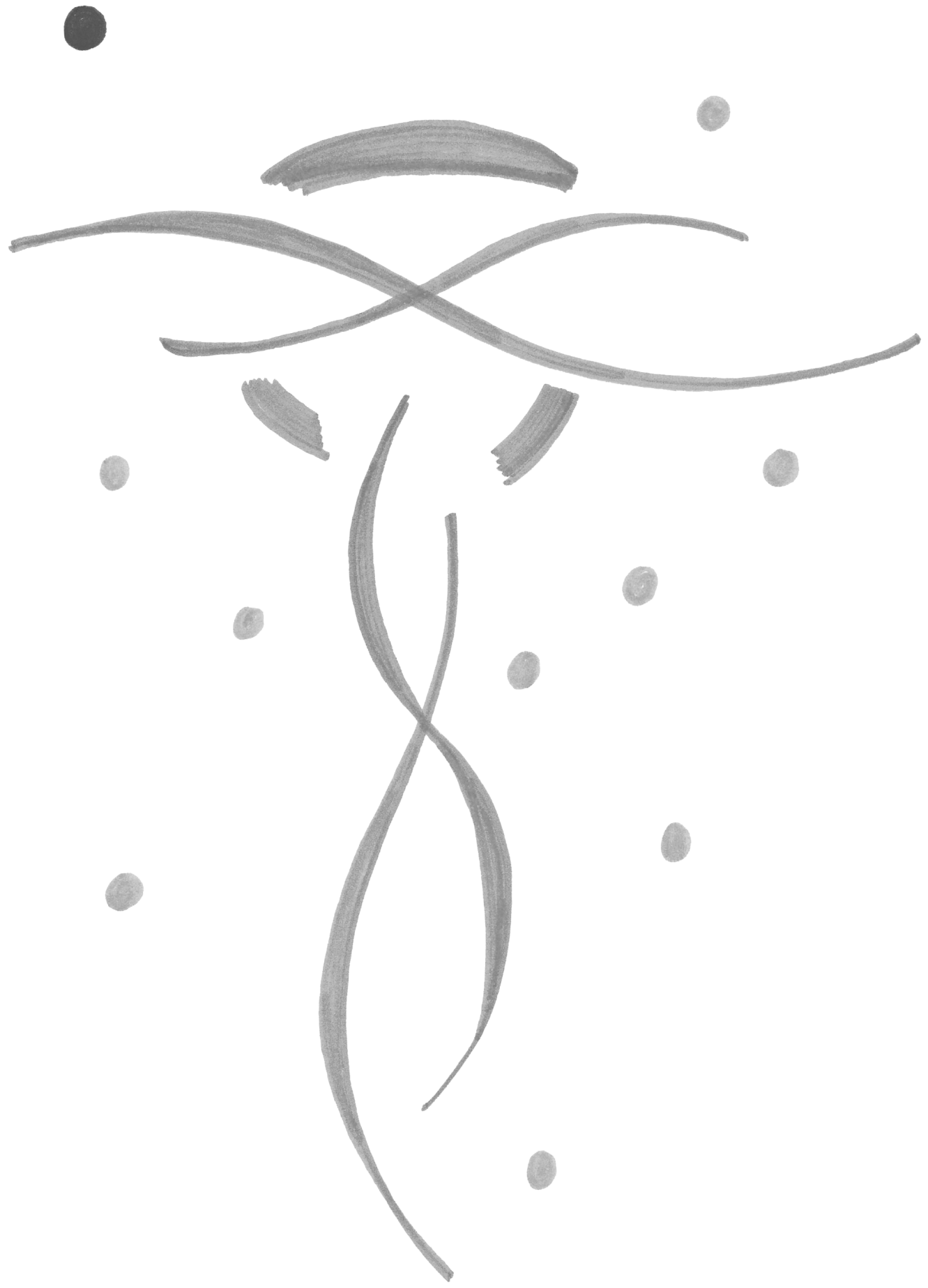




\section{Chapter 9}

\section{General discussion}




\section{General discussion}

The diagnosis early-stage ovarian cancer is based on the pathological results of a surgical staging procedure which consists of peritoneal washings for cytology, bilateral salginooophorectomy, hysterectomy, infracolic omentectomy, biopsy of the diaphragmatic peritoneum, paracolic gutters, pelvic peritoneum, and a systematic pelvic and para-aortic lymphadenectomy. Despite a clear recommendation by FIGO, the surgical staging of ovarian cancer differs greatly form centre to centre, especially with respect to the extent of lymph node dissection.

The main objectives of the studies in this thesis were:

- To assess the incidence of lymph node metastases in patients with clinical earlystage epithelial and non-epithelial ovarian cancer.

- To examine the possibility of identifying sentinel nodes in patients with ovarian cancer by injecting the tracers into the ovarian ligaments.

- To determine the impact of lymph node staging and adjuvant chemotherapy on the survival of patients with early-stage epithelial ovarian cancer.

\section{Main findings}

\section{Incidence and location of lymph node metastases in clinical early-stage ovarian cancer}

In a review, including 14 studies with a total of 361 patients, the mean incidence of lymph node metastases in clinical stage I and IIA invasive epithelial ovarian cancer was $14.2 \%$ (chapter two). In patients with lymph node metastases, $50 \%$ of the metastases were only detected in the para-aortic region, $20 \%$ only in the pelvic region, and the remaining $30 \%$ both in the para-aortic and pelvic region. Grade 1 tumours had a mean incidence of lymph node metastases of $4.0 \%$, grade 2 tumours $16.5 \%$, and grade 3 tumours $20.0 \%$. According to histological subtype, the highest incidence of lymph node metastases was found in the serous subtype (23.3\%), the lowest in the mucinous subtype $(2.6 \%)$.

For non-epithelial ovarian cancer, in case of sex cord stromal tumours no lymph-node metastases were detected in the 86 patients who underwent lymph-node removal (chapter three). In case of germ cell tumours the mean incidence of lymph-node metastasis in 946 patients was $11 \%$ with the highest incidence in patients with dysgerminoma of the ovary (18.1\%). 


\section{Sentinel nodes in ovarian cancer}

We performed a feasibility study to identify possible sentinel nodes by injecting tracers into the ovarian ligaments (chapter five). In all 21 patients one or more sentinel nodes were detected with the gamma probe. The locations of the hot spots correlated well with the locations of lymph node metastases in clinical stage I-II epithelial ovarian cancer. An anatomical study in 3 human female foetuses of the lymphatic drainage pathways of the ovaries confirmed the two major lymphatic drainage pathways from the ovaries (chapter six). One pathway drained the ovaries via the infundibulopelvic ligament (the suspensory ligament) towards the para-aortic and paracaval lymph nodes. Another pathway drained via the ovarian ligament (proper ligament) towards the lymph nodes in the obturator fossa and the internal iliac artery. A third minor pathway drained the ovaries via the round ligament to the inguinal lymph nodes. Lymph vessels draining the fallopian tube all followed the lymphatic drainage pathways of the ovaries.

\section{Impact of lymph node staging and adjuvant chemotherapy in early-stage epithelial ovarian cancer on survival}

In a nationwide retrospective cohort study we found that the relative survival of patients with removal of ten or more lymph nodes (including patients with lymph node metastases) was significantly better than of patients without, also after correction for FIGO stage, tumour grade, histology, and age (89\% and $82 \%$ respectively, relative excess risk (RER) 0.63, 95\% CI 0.52-0.78)(chapter seven).

Adjuvant chemotherapy after an adequate lymph node dissection did not contribute to a better relative survival, even in patients with high-risk features.

Furthermore, in patients without lymph node metastases, survival in early-stage epithelial ovarian cancer improved with the number of lymph nodes removed.

\section{Implications for clinical practise}

\section{Importance of lymph node staging}

Is restaging necessary?

Not all invasive malignant ovarian tumours are detected before or during surgery. In some patients who are expected to have a benign adnexal mass, only a salpingo-oophorectomy is performed, and the diagnosis of an invasive malignancy is made after surgery. In case of a suspected ovarian malignancy, frozen section of the adnexal mass during surgery can be 
false negative, either identifying a benign or borderline tumour. In case of a borderline tumour, the staging procedure does not include a lymph node dissection. In case of a malignancy the question is whether or not to perform a second surgical procedure to perform a complete staging procedure including a lymphadenectomy.

With a mean incidence of $14 \%$ lymph node metastasis in clinical early-stage invasive epithelial ovarian cancer, in general, a restaging procedure is necessary (chapter two). With regards to the differentiation grade, we found that the chance of finding lymph node metastases in grade 1 tumours was lowest with an incidence of $4.0 \%$. With regards to histological subtypes, we noticed that the incidence of positive lymph nodes was only $2.6 \%$ in mucinous tumours. Although we were not able to discern this figure from the literature, we can presume that the incidence of positive lymph nodes is even lower in a mucinous grade 1 tumour. The chance of finding lymph node metastases in grade I mucinous tumours is therefore slim. In this subgroup of patients it can be considered to omit a systematic lymphadenectomy when a restaging procedure is indicated due to the low incidence of positive lymph nodes and the considerable morbidity of a systematic pelvic and para-aortic lymphadenectomy. ${ }^{1}$

With regard to non-epithelial ovarian cancer, lymph node metastases were absent in clinical stage I-II sex cord stromal tumours (chapter three). Therefore a pelvic and paraaortic lymphadenectomy can be omitted in a sex cord stromal tumour.

In patients with a germ cell tumour lymph node metastases are not seldom. However, a recurrence or progression of a germ cell tumour in a metastatic lymph node is less severe since these tumours are extremely chemosensitive. Germ cell tumours are most often seen in young girls, surgery is often restricted to a unilateral adnexectomy combined with an intraoperative evaluation of the peritoneal cavity and biopsies of suspicious sites. How extensive should we perform a lymph node staging?

The minimum number of lymph nodes required for adequate lymph node staging remains controversial. We found that overall survival improved in line with the number of lymph nodes removed (chapter seven). This correlation was also observed by others, and is probably based on stage migration, i.e. patients with (occult) lymph node metastases progressing to advanced stage disease..$^{2-5}$ The current Dutch guideline recommends a minimum of ten lymph nodes to be removed from different anatomical regions. ${ }^{6}$ Based on 
our retrospective study an adequate dissection of at least 10 , but preferably 20 or more lymph nodes should be the standard procedure for the staging of early-stage epithelial ovarian cancer. During surgery it is difficult to estimate the number of lymph nodes removed, therefore enough perivascular tissue should be resected to make sure enough lymph nodes are removed.

With respect to the location of lymph node metastases, based on our review (chapter two), the absolute incidence of isolated pelvic lymph node metastases is approximately $3 \%$. In case of a unilateral invasive epithelial ovarian cancer, lymph node metastases can also occur at the contralateral pelvic site. In patients with lymph node metastases, approximately $16 \%$ occurs isolated on the contralateral pelvic site, meaning that the absolute incidence of isolated lymph node metastases on the contralateral pelvic site is below $1 \%$. One can argue that, based on such a low percentage, in patients with unilateral invasive epithelial ovarian cancer, pelvic lymph node dissection at the contralateral site can be omitted.

We were not able to explain the occurrence of contralateral pelvic lymph node metastases in our lymphatic ovarian drainage pathways study in human fetuses. We did not detect connections between the right and left lymphatic ovarian drainage pathways in the analyzed fetuses. But examining only three fetuses of course does not exclude that crossconnections exist.

Inguinal lymph node metastases in invasive epithelial ovarian cancer appear to occur even more rare than contralateral pelvic lymph node metastases. Based on our study of the lymphatic ovarian drainage pathways in three analyzed fetuses, the occurrence of inguinal lymph node metastases can be explained by a minor lymphatic drainage pathway via the round ligament.

\section{Chemotherapy in early-stage epithelial ovarian cancer}

Does chemotherapy corrects the worsened survival in case of an incomplete surgical staging, in particular no lymph node resection?

We found that adjuvant chemotherapy significantly improved the relative survival of patients that did not undergo lymph node staging. But patients from whom ten or more lymph nodes were removed as part of a staging procedure, and did not receive adjuvant 
chemotherapy, had a significantly better relative survival than patients without lymph node removal that did receive adjuvant chemotherapy (chapter seven). This remained true after correcting for age, differentiation grade, subtype histology and stage. These results could be construed as evidence supporting a therapeutic role for lymph node dissection. However, because we excluded patients with lymph node metastases from the group of patients that underwent lymph node dissection but no adjuvant chemotherapy, it is plausible that the survival benefit is explained by stage migration.

Does chemotherapy improve survival after an adequate lymph node resection in high-risk patients?

It is suggested that adjuvant chemotherapy after an adequate staging procedure should be considered for a small and selective group of high-risk patients (aneuploidy tumours and/or FIGO stage IC and IIA and/or tumour grade 3 and/or clear cell histology). ${ }^{7}$ The latter assumption is based on an analysis of high-risk patients in a retrospective study, as well as on extrapolation from experience in patients with advanced stage ovarian cancer. However, we found that high-risk patients (FIGO stage IC and IIA and/or tumor grade 3 and/or clear cell histology) from whom ten or more lymph nodes were removed as part of a staging procedure did not benefit from adjuvant chemotherapy (chapter seven). Since this cohort comprised 367 patients with high-risk features (244 with and 123 without adjuvant chemotherapy), this is the largest study to examine the influence of lymph node dissection and chemotherapy on survival. Chemotherapy after an adequate lymph node dissection does not seem to have an advantage on survival, however a prospective randomized trial for this specific high-risk group is needed.

\section{Sentinel node technique in ovarian cancer}

In breast cancer the sentinel node technique has been proven successful, and sentinel node studies in cervical and uterine cancers are currently under way. ${ }^{8-11}$ Sentinel node studies in ovarian cancer are scarce. Negishi et al. used injection of an activated charcoal solution into the ovarian capsule to identify sentinel nodes and reported the detection of sentinel nodes in the para-aortic region in all 11 patients analysed (overall eighteen sentinel nodes) and in the pelvic region in four patients (overall four sentinel nodes). ${ }^{12}$ Nyberg et al. analysed 16 patients with high-risk uterine cancer by injecting technetium and blue dye into the right or left ovary and detected sentinel nodes in 15 of the 16 
patients. ${ }^{13}$ The sentinel nodes $(n=30)$ were all located in the para-aortic region. The sentinel nodes related to the left ovary were mainly (64\%) located above the level of the inferior mesenteric artery, whereas most sentinel nodes of the right ovary (94\%) were found below the level of the inferior mesenteric artery, which is in agreement with our findings. In both studies, the tracer was injected into the ovary. However, the injection of tracers into the ovary can be difficult when bulky ovarian masses are present. Furthermore, the injection of tracers into the ovarian capsule is associated with a potential risk of tumour dissemination. ${ }^{10-11}$ Although the effect on prognosis of peroperative spill still is controversial, current practice by the FIGO guidelines is to avoid spillage. ${ }^{14}$ This probably explains why, hitherto, sentinel node detection in ovarian cancer has not been adopted in clinical practice

\section{Injection of the tracers in the ligaments}

We showed that perioperative injection of tracers into the ovarian ligaments is a feasible method for the detection of sentinel nodes (chapter five). One or more sentinel nodes were detected with the gamma probe in all the patients $(n=21)$ who underwent the sentinel node procedure. Hot spots were found isolated in the para-aortic and para-caval regions in most patients (67\%), in the pelvic region only in $9 \%$, and in both the paraaortic/para-caval and pelvic regions in $24 \%$ of the patients. In all but two patients, the sentinel nodes were ipsilateral to the injection site. These findings correlate well with the results of chapter two on the incidence and location of lymph node metastases in clinical stage I-II epithelial ovarian cancer, in which metastases were found isolated in the paraaortic region in $50 \%$, isolated in the pelvic region in $20 \%$, and in both the para-aortic and pelvic region in the remaining $30 \%$ of patients.

Of course, this feasibility study only shows that lymph nodes can be detected by injecting the tracers into the ovarian ligaments. More questions need to be answered before this technique can be used in daily clinical practice. This is discussed in the paragraph with suggestions for future research.

\section{Future directions}

Although doing research leads to answers it also leads to more questions. I have several suggestions for future research. 


\section{Improving peroperative detection of sentinel nodes}

In general, lymphatic mapping involves the injection of a blue dye or a radioactive isotope. Sentinel node studies in different primary tumours showed that the detection rate is highest when a radioactive isotope and the blue dye are used in combination. ${ }^{15-16}$ In our feasibility study (chapter five), blue-stained sentinel nodes were identified in only two out of six patients during retroperitoneal exploration. The long interval between the injection of the tracer and retroperitoneal exploration of at least 50 minutes may have caused the blue dye to fade before the surgical procedure. A sentinel node study in cervical cancer reported the detection of blue-stained lymph nodes after a median time of 7 minutes after injection, with the lymph nodes remaining blue for a median time of 21 minutes. ${ }^{17}$ In future studies the utility of the blue dye as a tracer in case of ovarian cancer should be examined in more detail.

The exact timing for the detection of positive lymph nodes remains undefined. The time between injection and detection should be long enough to allow the tracers to be transported to the sentinel nodes, but an increased lag time requires prolonged general anaesthesia and increased costs. Therefore, delaying surgery for a period longer than 15 minutes was deemed not to be feasible in daily practice. In the feasibility study in chapter six, we used a 15-minute interval between injection of the tracers and resection of the adnexal mass, and at least one sentinel node was identified in all patients within this period. In case of an ovarian malignancy, the time interval between injection of the tracers and detection and resection of the sentinel node(s) was at least 50 minutes (including 15 minutes waiting time after injection, and time spent for removal of the ovarian tumour and frozen section analysis). As described in the results, in four patients additional sentinel nodes within the same region were discovered after opening of the retroperitoneum and in three patients additional sentinel nodes were discovered in other regions. Identifying more lymph nodes during retroperitoneal exploration could be explained by a better and more precise accessibility of the lymph node locations but possibly also because of a longer time interval after injection of the tracer.

The optimal colloid particle sizes, which result in the fastest migration and best retention in sentinel nodes, should also be determined. In a study by Glass et al., examining the kinetics of three different radioactive agents in cutaneous melanoma, detection at 30 
minutes was more appropriate than late imaging. ${ }^{18}$ Paganelli et al. studying breast cancer, showed that the number of sentinel nodes detected strongly related to the size of the particle, i.e. the smaller the particles, the greater the number of lymph nodes. ${ }^{19}$ However, all these data may be organ dependent, so it is presently unknown if this is also the case in ovarian cancer. Moreover, newly developed hybrid sentinel node detection agents, combining radioactivity with fluorescence and/or paramagnetic capabilities, alternative operative techniques such as multiphased injection, and new intraoperative imaging technologies may provide additional advantages..$^{20-23}$

Preoperative SPECT/CT has been found to maximize the accuracy of sentinel node detection in cervical and uterine malignancy. ${ }^{24}$ Therefore, the nature of the approach using a gamma probe to identify hot spots during surgery without pre-operative imaging of the sentinel node localizations, implies that sentinel nodes might be missed. A technique that may be of interest is the intra-operative use of a portable gamma camera with a large diameter scintillation crystal, which may result in real time imaging. ${ }^{25}$ This method may provide an overview of all radioactive hotspots throughout the entire surgical field. A study in breast cancer patients showed that visualization of sentinel nodes with a portable gamma camera was similar to that with a conventional gamma camera. ${ }^{26}$ For ovarian tumours, an overview of all sentinel nodes with the portable gamma camera, together with a hand-held probe, may provide more certainty regarding the completeness of sentinel node excision.

\section{Sentinel node detection after removal of the ovarian tumour}

In our feasibility study in which tracers were injected into the ovarian ligaments to identify possible sentinel nodes, the injection occurred before the adnex was resected for frozen section. This sequence was chosen because we hypothesized that shortly after resection, the lymph flow in the remnants of the ligaments might be significantly reduced. However, this means that in a significant number of patients the injection of tracers was unnecessary since the result of frozen section identified a benign of borderline lesion for which no lymph node resection is necessary.

It should be investigated whether or not the lymph flow is reduced immediately after a salpingo-oophprectomy, in such a way that no sentinel node can be identified. 
Not all invasive malignant ovarian tumours are detected before or during surgery, either because a patient undergoes conservative surgery for what is thought to be a benign ovarian tumour, or frozen section is falsely negative. These patients should be planned for a second surgery in which a staging procedure is done. It should be investigated if indeed, a few weeks after a salpingo-oophoprectomy, the lymph flow is sufficient to identify sentinel nodes after injection of the tracers in the remnants of the ovarian ligaments. The current study was done by laparotomy. If the same sentinel node procedure performed by laparoscopy is feasible, for example in patients in whom a second surgical procedure is necessary to complete the staging procedure, has not been investigated so far.

\section{Cost-effectiveness of the sentinel node technique}

The main purpose of the sentinel node technique in clinical early-stage ovarian cancer is the reduction of treatment-related morbidity in patients without metastatic lymph nodes while avoiding the risk of missing involved lymph nodes. Approximately $14 \%$ of patients with clinical early-stage EOC have lymph node metastases (chapter two). As a consequence, $86 \%$ of patients are not likely to benefit from a lymphadenectomy, although they are at risk for surgery-related morbidity.

In patients with a complex adnexal mass, the sentinel node procedure should be performed before the diagnosis epithelial ovarian cancer can be made based on frozen section. Not all patients prove to have a malignant ovarian tumour. As a consequence approximately $40 \%$ of the patients receive the sentinel node tracers while having a benign adnexal mass. ${ }^{27}$ Therefore the added costs of sentinel node procedures in patients with a benign ovarian tumour should be taken into account. For these patients the sentinel node procedure does not give any advances, however the risk of complications and side effects of the procedure is low. ${ }^{28-30}$

On the other hand for patients with invasive epithelial ovarian cancer and lymph node metastases the use of sentinel node procedure as a substitute for a lymph node sampling may even be associated with a higher sensitivity for the identification of involved lymph nodes. 


\section{References}

1. Benedetti Panici P, Maggioni A, Hacker N, Landoni F, Ackermann S, Campagnutta E, Tamussino K, Winter R, Pellegrino A, Greggi S, Angioli R, Manci N, Scambia G, Dell'Anna T, Fossati R, Florani I, Rossi RS, Grassi R, Favalli G, Raspagliesi F, Giannarelli D, Martella L, Mangioni C. Systematic aortic and pelvic lymphadenectomy versus resection of bulky nodes only in optimally debulked advanced ovarian cancer: a randomized clinical trial. J Natl Cancer Inst 2005;97:560- 566 .

2. Maggioni A, Benedetti Panici P, Dell'Anna T, Landoni F, Lissoni A, Pellegrino A, Rossi RS, Chiari S, Campagnutta E, Greggi S, Angiolo R, Manci N, Calcagno M, Scambia G, Fossati R, Floriani I, Torri, Grassi R, Mangioni C. Randomised study of systematic lymphadenectomy in patients with epithelial ovarian cancer macroscopically confined to the pelvis. Br J Cancer 2006;18:699-704.

3. Cress RD, Bauer K, O’Malley CD Kahn AR, Schymura MJ, Stewart SL, Leiserowitz GS. Surgical staging of early-stage epithelial ovarian cancer: results from the CDC-NPCR ovarian patterns of care study. Gynecol Oncol 2011;121:94-99.

4. Carnino F, Fuda G, Ciccone G, Iskra L, Guercio E, Dadone D, Conte PF. Significance of lymph node sampling in epithelial carcinoma of the ovary. Gynecol Oncol 1997;65:467-472.

5. Chan JK, Munro EG, Cheung MK, Husain A, Teng NN, Berek JS, Osann K. Association of lymphadenectomy and survival in stage I ovarian cancer patients. Obstet Gynecol 2007;109:12-19.

6. http://www.oncoline.nl/ovariumcarcinoom

7. Vergote I, De Brabanter J, Fyles A, Bertelsen K, Einhorn N, Sevelda P, Gore ME, Kaern J, Verreist H, Sjövall K, Timmerman D, Vandewalle J, Van Gramberen M, Tropé CG. Prognostic importance of degree of differentiation and cyst rupture in stage I invasive epithelial ovarian carcinoma. Lancet 2001 357:176-182,

8. de Hullu JA, Hollema H, Piers DA, Verheijen RH, van Diest PJ, Mourits MJ, Aalders JG, van Der Zee AG. Sentinel lymph node procedure is highly accurate in squamous cell carcinoma of the vulva. J Clin Oncol 2000;18:2811-2816.

9. Mansel RE, Fallowfield L, Kissin M, Goyal A, Newcombe RG, Dixon JM, Yiangou C, Horgan K, Bundred N, Monypenny I, England D, Sibbering M, Abdullah TI, Barr L, Chetty U, Sinnett DH, Fleissig A, Clarke D, Ell PJ. Randomized multicenter trial of 
sentinel node biopsy versus standard axillary treatment in operable breast cancer: the ALMANAC Trial. J Natl Cancer Inst 2006;98:599- 609.

10. El-Ghobashy AE, Saidi SA. Sentinel node sampling in gynaecological cancers: techniques and clinical applications. Eur J Surg Onc 2009;35:675-685.

11. Giammarile F, Vidal-Sicart S, Valdés Olmos RA. Uncommon applications of sentinel lymph node mapping: urogenital cancers. Q J Nucl Med Mol Imaging 2014;58:161179.

12. Negishi H, Takeda M, Fujimoto T, Todo Y, Ebina Y, Watari H, Yamamoto R, Minakami H, Sakuragi N. Lymphatic mapping and sentinel node identification as related to the primary sites of lymph node metastasis in early stage ovarian cancer. Gynecol Oncol 2004;94:161-166.

13. Nyberg RH, Korkola $P$, Tech L, Mäenpää J. Ovarian sentinel node, is it feasible? Int J Gynecol Cancer 2011;21:567-572.

14. Prat J, Mutch DG. 2014 FIGO staging for ovarian, fallopian tube and peritoneal cancer. Gynecol Oncol 2014;133:401-404.

15. Hayashida T, Jinno H, Sakata M, Takahasi M, Onishi M, Seki H. Superiority of radioactive over blue dye for sentinel lymph node detection in breast cancer. Eur Surg Res 2010;44:111-116.

16. Eiriksson LR, Covens A. Sentinel lymph node mapping in cervical cancer: the future? BJOG 2012;119:129-133.

17. Levenback C, Coleman RL, Burke TW, Lin WM, Erdman W, Deavers M, Delpassand ES. Lymphatic mapping and sentinel node identification in patients with cervix cancer undergoing radical hysterectomy and pelvic lymphadenectomy. J Clin Oncol 2002;20:688-693.

18. Glass EC, Essner R, Morton DL. Kinetics of three lymphoscintigraphic agents in patients with cutaneous melanoma. J Nucl Med 1998;39:1185-1190.

19. Paganelli G, De Cicco C, Cremonesi M, Prisco G, Calza P, Luini A, Zucali P, Veronesi U. Optimized sentinel node scintigraphy in breast cancer. Q J Nucl Med 1998;42:4953.

20. Ahmed M, Purushotham AD, Douek M. Novel techniques for sentinel lymph node biopsy in breast cancer: a systematic review. Lancet Oncol 2014;15:351-362. 
21. Tsuchimochi M, Hayama K. Intraoperative gamma cameras for radioguided surgery: technical characteristics, performance parameters, and clinical applications. Phys Med 2013;29:126-138.

22. Schaafsma BE, van der Vorst JR, Gaarenstroom KN, Peters AA, Verbeek FP, de Kroon CD, Trimbos JB, van Poelgeest MI, Frangioni JV, van de Velde CJ, Vahrmeijer AL Randomized comparison of near-infrared fluorescence lymphatic tracers for sentinel lymph node mapping of cervical cancer. Gynecol Oncol 2012;127:126-130.

23. Schaafsma BE, Verbeek FP, Peters AA, van der Vorst JR, de Kroon CD, van Poelgeest MI, Trimbos JB, van de Velde CJ, Frangioni JV, Vahrmeijer AL, Gaarenstroom KN. Near-infrared fluorescence sentinel lymph node biopsy in vulvar cancer: a randomised comparison of lymphatic tracers. BJOG 2013;120:758-764.

24. Pandit-Taskar, N, Gemignani ML, Lyall A, Larson SM, Barakat RR, Abu Rustum NR. Single photon emission computed tomography SPECT-CT improves sentinel node detection and localization in cervical and uterine malignancy. Gynecol Oncol 2010;117:59-64.

25. Vermeeren, L, Meinhardt W, Bex A, van der Poel HG, Vogel WV, Hoefnagel CA, Horenblas S, Valdés Olmos RA. Paraaortic sentinel lymph nodes: toward optimal detection and intraoperative localization using SPECT/CT and intraoperative realtime imaging. J Nucl Med 2010;51:376-382.

26. Vidal-Sicart, S, Vermeeren L, Solà O, Paredes P, Valdés-Olmos RA. The use of a portable gamma camera for preoperative lymphatic mapping: a comparison with a conventional gamma camera. Eur J Nucl Med Mol Imaging 2011;38:636-641.

27. Geomini P, Kruitwagen RFPM, Bremer GL, Cnossen J, Mol BW. The accuracy of risk scores in predicting ovarian malignancy: a systematic review. Obstet Gynecol 2009;113:384-394.

28. Bézu C, Coutant C, Salengro A, Daraï E, Rouzier R, Uzan S. Anaphylactic response to blue dye during sentinel node biopsy. Surg Onc 2011;20:55-59.

29. Dewachter P, Mouton-Faicre C, Trechot P, Lleu JC, Mertes PM. Severe anaphylactic shock with methylene instillation. Anesth Analg 2005;101:149-150.

30. Rzymski P, Wozniak J, Opala T, Wilczak M, Sajdak S. Anaphylatic reaction to methylene blue dye after laparoscopic chromopertubation. Int J Gynaecol Obstet 2003;81:71-72. 


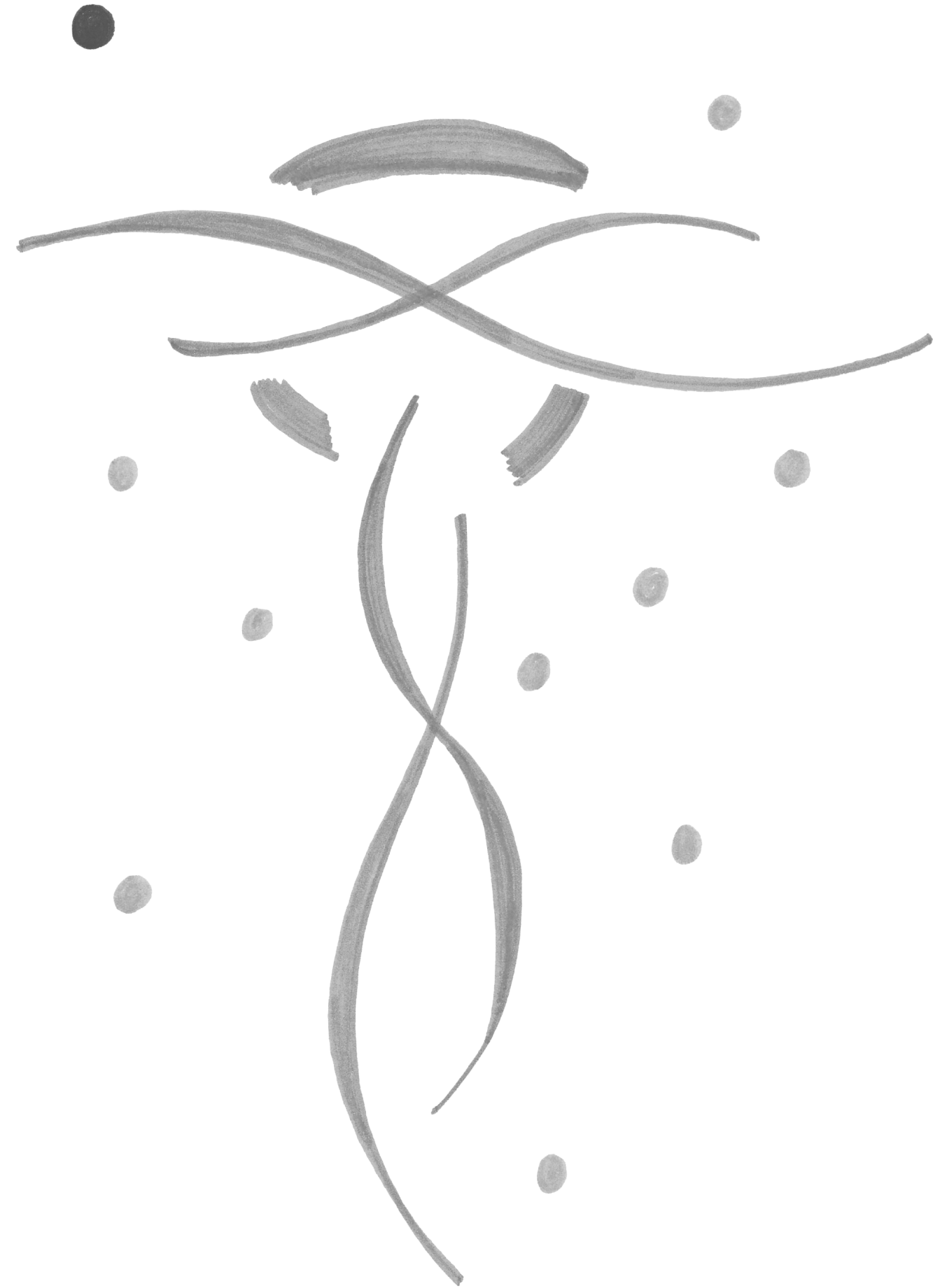




\section{Chapter 10}

Summary 


\section{Summary}

Chapter one gives a general introduction as well as the outline of this thesis. Ovarian cancer is the tumour with the most unfavourable prognosis within the field of gynaecological oncology. The high mortality rate is mainly due to the fact that approximately $75 \%$ of the patients are diagnosed with advanced stage epithelial ovarian cancer (EOC). According to the International Federation of Gynaecology and Obstetrics (FIGO), EOC with lymph node metastases is classified as advanced FIGO stage IIIA1 disease for which adjuvant chemotherapy is indicated. In contrast, at least for the majority of patients with early-stage disease that are adequately staged, adjuvant chemotherapy is not indicated. Therefore, recognition of lymph node metastases is of utmost importance to determine the correct FIGO stage and providing the appropriate treatment.

Chapter two describes a review on the incidence of lymph node metastases in clinical stage I and II EOC. Included studies were prospective or retrospective cohort studies, which analyzed patients with clinical early-stage EOC who underwent a complete pelvic and para-aortic lymphadenectomy as a part of a staging laparotomy.

Fourteen studies were included in the analysis. The mean incidence of lymph node metastases in clinical stage I-II EOC was $14.2 \%$ (range $6.1-29.6 \%$ ), of which $6.7 \%$ only in the para-aortic region, $3.3 \%$ only in the pelvic region, and $4.3 \%$ both in the para-aortic and pelvic region. Grade 1 tumours had a mean incidence of lymph node metastases of $4.0 \%$, grade 2 tumours $16.5 \%$ and grade 3 tumours 20.0\%. According to histological subtype, the highest incidence of lymph node metastases was found in the serous subtype (23.3\%), the lowest in the mucinous subtype (2.6\%). In unilateral tumours, pelvic lymph node metastases were found in $9.7 \%$ on both sides, $8.3 \%$ only at the ipsilateral side, and in $3.5 \%$ only at the contralateral side.

It is concluded that the incidence of lymph node metastases in clinical early-stage EOC is considerable and concerns both the para-aortic and pelvic lymph nodes. Based on the scarce literature data, omitting a systematic lymphadenectomy can only be considered in grade I mucinous tumours.

Chapter three describes a review on the incidence of lymph node metastasis in clinical stage I and II non-EOC (sex cord stromal tumours and germ cell tumours). Included 
studies were prospective or retrospective cohort and cross-sectional studies analysing at least ten patients with clinical early-stage non-EOC who underwent lymphadenectomy or lymph-node sampling as part of a staging laparotomy.

For sex cord stromal tumours, five articles including 578 patients were analysed. No lymph-node metastases were detected in the 86 patients who underwent lymph-node removal. The median number of removed lymph nodes was 13 (range 9-29). For malignant germ cell tumours, four articles were eligible including 2436 patients of whom 946 patients underwent lymph-node resection. The mean number of removed nodes was 10 (range 2-14) with a mean incidence of lymph-node metastasis of $10.9 \%$ (range 10.5-11.8\%).

It is concluded that the incidence of lymph-node metastasis in patients with clinical stage I and II sex cord stromal tumours is low, whereas the incidence in patients with clinical stage I-II germ cell tumours is considerable, although limited data are available.

Chapter four gives the study protocol for our feasibility study for a sentinel node (SN) procedure in early-stage ovarian cancer. The concept of sentinel lymph node surgery is to determine whether the cancer has spread to the very first lymph node or SN. If the SN does not contain cancer, then there is a high likelihood that the cancer has not spread to other lymph nodes. The SN technique has been proven to be effective in different types of cancer. Patients with a high suspicion of an ovarian malignancy in whom a median laparotomy and a frozen section analysis is planned were eligible.

Before resecting the pathological adnex, blue dye and radioactive-colloid was injected in the ligamentum ovarii proprium and the ligamentum infundibulo-pelvicum followed by a 15 minutes time-interval. By injection the tracers in the ovarian ligaments instead of the ovarian cortex, possible spillage of tumourcells is avoided. The primary outcome was the percentage of patients in whom it is feasible to identify SNs. Another study parameter was the anatomical localisation of the SNs.

The results of the feasibility study are described in chapter five. A SN procedure was performed in 21 patients and at least one SN location was identified in all patients using the gamma probe before retroperitoneal exploration. SNs were located in the para-aortic and para-caval regions only in $67 \%$ of the patients, in the pelvic region only in $9 \%$, and in both the para-aortic/para-caval and pelvic regions in $24 \%$ of patients. All but two SNs 
were found on the ipsilateral side. In six patients who underwent retroperitoneal exploration, one to four SNs were identified using the gamma probe and resected. Bluestained SNs were detected only in two patients. Positive SNs were detected in one patient with lymph node metastases. The amount of radiation exposed to the surgeon, theatre nurse and pathologist did not exceed the safe limit.

Chapter six describes the lymphatic drainage pathways of the ovary. Up to now, studies reporting the lymphatic drainage pathways of the ovaries on microscopic level are lacking. A series of three human female foetuses and tissues samples from one human cadaveric specimen were studied. Immunohistochemical analysis was performed on paraffin-

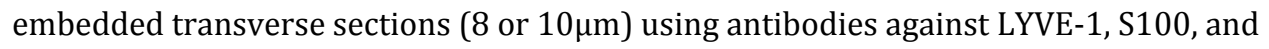
alpha-smooth muscle actin to identify the lymphatic endothelium, Schwann and smooth muscle cells, respectively. Two major and one minor lymphatic drainage pathway from the ovaries were detected. One pathway drained via the ovarian ligament towards the lymph nodes in the obturator fossa and the internal iliac artery. Another pathway drained the ovaries via the infundibulopelvic ligament towards the para-aortic and paracaval lymph nodes. A third minor pathway drained the ovaries via the round ligament to the inguinal lymph nodes. Lymph vessels draining the fallopian tube all followed the lymphatic drainage pathways of the ovaries.

Chapter seven describes a nationwide cohort study to establish the impact of lymph node resection and chemotherapy on the survival in 3658 patients with early-stage EOC. All patients with FIGO stage I-IIA and IIIA1 EOC in the Netherlands between 2000 and 2012 were included. Data concerning age, FIGO stage, differentiation grade, histological subtype, hospital type, number of lymph nodes removed, whether or not adjuvant chemotherapy was given, and survival data were extracted from the Netherlands Cancer Registry. A total of 1813 patients (49.6\%) had lymph nodes removed as part of the surgical staging procedure. The relative survival of patients with removal of lymph nodes (including patients with lymph node metastases) was significantly better than of patients without, also after correction for FIGO stage, tumour grade, histology, and age (89\% and $82 \%$ respectively, relative excess risk (RER) $0.63,95 \%$ CI 0.52-0.78). A significant correlation was found between the number of lymph nodes removed and overall survival. However, above twenty lymph nodes the survival benefit did not increase any further. 
In patients with a lymph node dissection of $\geq 10$ lymph nodes no difference was found in relative survival in the group without chemotherapy compared to the group with chemotherapy (RER 0.62, 95\% CI 0.24- 1.62). This also applied for the subgroup of patients with the high-risk features: FIGO stage IC and IIA and/or differentiation grade 3 and/or clear cell histology (RER 1.00, 95\% CI 0.49- 2.09).

In early-stage EOC, adjuvant chemotherapy after lymph node dissection of ten or more lymph nodes does not seem to contribute to a better relative survival, even in patients with high-risk features. Therefore, adequate dissection of at least 10, but preferably 20 of more lymph nodes should be the standard procedure for the staging of early-stage EOC.

Chapter eight contains a general discussion. Conclusions of this thesis, and wider implications and suggestions for further research are given.

Chapter nine gives a summary, a Dutch version is included. 


\section{Samenvatting}

Hoofdstuk één geeft een algemene introductie met daarbij een uiteenzetting van dit proefschrift. Het ovariumcarcinoom is binnen de gynaecologische oncologie de tumor met de slechtste prognose. De hoge mortaliteit is grotendeels te wijten aan het feit dat ongeveer $75 \%$ van de patiënten gediagnosticeerd wordt met een vergevorderd stadium epitheliaal ovariumcarcinoom (EOC). Binnen de classificatie volgens de Internationale Federatie van Gynaecologie en Obstetrie (FIGO), wordt EOC met lymfkliermetastasen geclassificeerd als FIGO stadium IIIA1, ook bij afwezigheid van peritoneale metastasen. Voor deze patiënten is adjuvante chemotherapie geïndiceerd. Dit in tegenstelling tot de patiënten met een vroeg stadium EOC, wanneer zij adequaat gestadiëerd zijn, is adjuvante chemotherapie niet geïndiceerd. Daarom is het van groot belang om lymfkliermetastasen te herkennen. Zodat patiënten in het juiste FIGO stadium worden geplaatst en daarmee de juiste behandeling ontvangen.

In hoofdstuk twee beschrijven we de resultaten van een review om vast te stellen wat de incidentie is van lymfkliermetastasen in een klinisch vroeg stadium EOC. Prospectieve en retrospectieve cohort studies zijn geïncludeerd, waarbij patiënten werden geanalyseerd met een klinisch vroeg stadium epitheliaal ovariumcarcinoom, met een complete pelviene en para-aoartale lymfadenectomie als onderdeel van een stadiëringslaparotomie. Er werden veertien studies geïncludeerd. De gemiddelde incidentie van lymfkliermetastasen in FIGO stadium I-II EOC was 14.2\% (range 6.1-29.6\%), hiervan werd 6.7\% alleen paraaortaal gevonden, $3.3 \%$ alleen pelvien en $4.3 \%$ in beide regio's. De incidentie van lymfkliermetastasen in graad 1 tumoren was $4.0 \%$, graad 2 tumoren $16.5 \%$ en $20.0 \%$ in graad 3 tumoren. Wat betreft het histologische type zagen we de hoogste incidentie bij sereuze tumoren (23.3\%), het laagste bij het mucineuze type (2.6\%). Bij unilaterale tumoren werden pelviene metastasen aan beide kanten gevonden in 9.7\%, alleen aan de aangedane zijde in $8.3 \%$, en in $3.4 \%$ alleen aan de contralaterale zijde. We kunnen concluderen dat de incidentie van lymfkliermetastasen in klinisch vroeg stadium EOC aanzienlijk is en zowel gevonden wordt in de para-aortale en pelviene lymfklieren. Gebaseerd op de beperkte literatuur zou een systmatische lymfadenectomie achterwege gelaten kunnen worden in graad 1 mucineuse tumoren. 
In hoofdstuk drie bespreken we een review welke de incidentie van lymfkliermetastasen laat zien in klinisch stadium I-II sexcord-stromaceltumoren en maligne kiemceltumoren van het ovarium. De geïncludeerde studies zijn prospectieve en retrospectieve cohort studies en cross-sectional studies die minstens tien patiënten analyseerden die een lymfadenectomie of een lymfkliersampling ondergingen als onderdeel van een stadiëringslaparotomie. Voor de sexcord-stromalceltumoren werden 5 artikelen geïncludeerd met in totaal 578 patiënten, waarbij in 86 patiënten lymfklieren werden verwijderd. Bij geen van deze patiënten werden lymfkliermetastasen gevonden. Het gemiddeld aantal verwijderde lymfklieren was 13 (range 9-29).

Voor maligne kiemceltumoren werden vier artikelen geïncludeerd met daarin 2436 patiënten, waarbij in 946 patiënten lymfklieren werden verwijderd. Het gemiddeld aantal verwijderde lymfklieren bedroeg 10 (spreiding 2-14). In 10.9\% (spreiding 10.5-11.8\%) werden lymfkliermetastasen gevonden. De incidentie van lymfkliermetastasen van patiënten met een klinisch stadium I-II sexcord-stromaceltumor is laag, echter de incidentie van lymfkliermetastasen bij patiënten met maligne kiemceltumoren is aanzienlijk. Maar dit is gebaseerd op slechts een beperkte hoeveelheid beschikbare data.

Hoofdstuk vier geeft een overzicht van het studie protocol van het haalbaarheidsonderzoek voor een sentinel node (SN) procedure bij het vroeg stadium ovariumcarcinoom. Het doel van de SN chirurgie is om te bepalen of de kanker zich heeft uitgebreid naar de eerst volgende lymfklier, de SN. Als de SN geen kanker bevat, dan is het zeer waarschijnlijk dat de kanker zich niet heeft uitgebreid naar de verdere lymfklieren. Deze SN techniek is bewezen effectief in andere soorten kanker. Patiënten met een hoge verdenking op een maligniteit van het ovarium waarvoor mediane laparotomie met vriescoupe, werden geïncludeerd. Voor het starten van de chirurgische stadiëringsprocedure, werden blauwe kleurstof en radioactief colloid geïnjecteerd in het ligamentum ovarium proprium en het ligamentum infundibulo-pelvicum. De primaire uitkomstmaat betrof het percentage van patiënten waarbij SN(s) werden herkend. Een secundaire uitkomstmaat was de anatomische locaties van de SN(s).

De resultaten van de studie naar de uitvoerbaarheid van een SN procedure in vroeg stadium ovariumcarcinoom worden gepresenteerd in hoofdstuk vijf. De SN procedure 
werd verricht in 21 patiënten, waarbij tenminste één SN lokalisatie herkend werd in elke patiënt met behulp van een gammaprobe voor het openen van het retro-peritoneum. De SNs waren in 67\% gelokaliseerd in de para-aortale en para-cavale regio, in $9 \%$ in de pelviene regio en in 24\% zowel para-aortaal/para-cavaal en pelvien. Alle SNs, behalve twee, werden gevonden aan de zelfde zijde als van de tumor. In zes patiënten die retroperitoneale exploratie ondergingen werden één tot vier SNs gevonden met de gammaprobe en vervolgens verwijderd. Blauw verkleurde lymfklieren werden slechts bij twee patiënten herkend. Positieve SNs werden gevonden in één patiënt met lymfklier metastasen. De hoeveelheid straling waaraan de gynaecologisch oncoloog, operatie assistent en de patholoog werden bloot gesteld bleeg binnen de gestelde veiligheidsnormen.

In hoofdstuk zes is de lymfdrainage van het ovarium onderzocht. Tot nog toe is verrassend genoeg de lymfedrainage van het ovarium slechts zeer beperkt onderzocht. Om de locaties van de SNs te kunnen voorspellen is het van belang om de routes van lymfdrainage van het ovarium te weten. Een serie van drie menselijke vrouwelijke foetus en een weefsel preparaat van een vrouwelijk volwassen kadaver werden bestudeerd. Immunohistochemische analyse werd uitgevoerd op een paraffine ingesloten transverse coupes ( 8 of $10 \mu \mathrm{m})$, waarbij gebruikt werd gemaakt van antilichamen tegen LYVE-1, S100 en alpha-gladde spier actine om het lymfatisch endotheel, Schwann en gladde spiercellen te identificeren. Twee belangrijke lymfdrainage routes en één minder uitgesproken lymfdrainage route werden ontdekt. Er was een route via het ligamentum ovarium proprium, naar de lymfklieren in de obturator fossa en de arterie illiaca externa. Een andere belangrijke route liep van het ovarium naar het ligamentum infundibulopelvicum naar de para-aortale en para-cavale lymfklieren. Een derde maar minder prominente route werd gevonden van het ovarium naar het ligamentum rotundum en de inguinale lymfklieren. De lymfvaten die tuba draineerden volgden dezelfde route als die van het ovarium.

In hoofdstuk zeven werd een nationale cohort studie verricht om vast stellen wat de impact is van lymfklierresectie en chemotherapie op het verdere klinische beleid en overleving bij 3658 patiënten met een vroeg stadium EOC. Alle patiënten met een FIGO stadium I-IIA en IIIA1 EOC in Nederland tussen 2000 en 2012 werden geïncludeerd. De 
volgende gegevens werden verkregen uit de Nederlandse Kanker Registratie: leeftijd, FIGO stadium, differentiatie graad, histologisch subtype, type ziekenhuis, aantal verwijderde lymfklieren, of er wel of niet adjuvante chemotherapie werd gegeven en overlevingsdata. In 1813 (49.6\%) patiënten werden lymfklieren verwijderd als onderdeel van de stadiëring. De relatieve overleving van patiënten waarbij lymfklieren werden verwijderd (inclusief de patiënten met positieve klieren) was significant beter dan bij patiënten waarbij geen lymfklieren werden verwijderd, ook na correctie voor FIGO stadium, gradering, histologie en leeftijd (89\% en 82\% respectievelijk, RER 0.63, 95\% CI 0.52-0.78). Een significante relatie werd gevonden tussen het aantal verwijderde lymfklieren en de overall overleving. Echter boven de 20 lymfklieren werd geen effect meer gezien op de overleving. In patiënten met een lymfklierdissectie van 10 lymfklieren of meer er was geen verschil gevonden in relatieve overleving tussen de groep die chemotherapie kreeg en de groep zonder chemotherapie (RER 0.62, 95\% CI 0.24- 1.62). Dit gold ook voor de groep met hoog risico kenmerken: FIGO IC en IIA en/ of graad 3 en/ of clear cell histologie (RER 1.00, 95\% CI 0.49-2.09).

In vroeg stadium EOC, adjuvante chemotherapie na lymfklierdissectie van tien of meer lymfklieren leek niet bij te dragen aan een betere overleving, zelfs niet in de patiënten met hoog risico kenmerken. Een adequate lymfklierdissectie van minimaal 20 lymfklieren zou een standaard procedure moeten worden in de stadiëring van vroeg stadium EOC.

Hoofdstuk acht bevat een algemene discussie. Besproken worden de conclusies van dit proefschrift, een breder perspectief en suggesties voor toekomstig onderzoek.

Hoofdstuk negen bevat een samenvatting in het Engels en het Nederlands. 


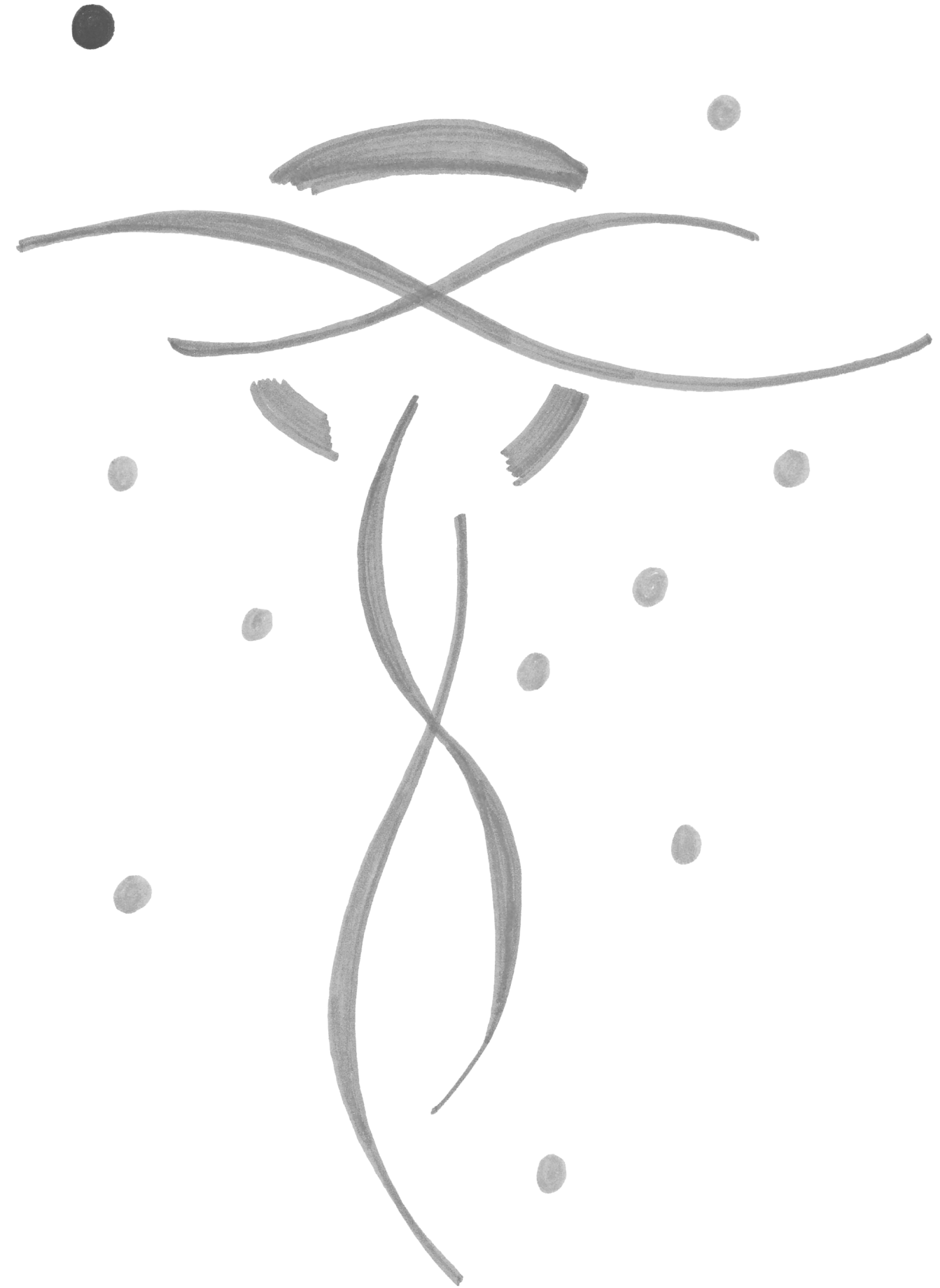




\title{
Chapter 11
}

\author{
List of co-authors \\ List of publications \\ Curriculum Vitae \\ Dankwoord
}




\section{Co-authors and their affiliations}

Dr. M.A. van der Aa, Netherlands Comprehensive Cancer Organisation, Utrecht, the Netherlands.

Drs. L.C.M. Amkreutz, Maastricht University Medical Centre, Department of Obstetrics and Gynaecology, Maastricht, The Netherlands.

Dr. B. Brans, University Hosipital Gent, Department of Nuclear Medicine, Gent, Belgium.

Dr. T. Van Gorp, Maastricht University Medical Centre, Department of Obstetrics and Gynaecology, GROW- School for Oncology and Developmental Biology, Maastricht, The Netherlands.

Dr. A.C. Kraima, Flevoziekenhuis, Department of Surgery, Almere, The Netherlands.

Prof. Dr. R.F.P.M. Kruitwagen, Maastricht University Medical Centre, Department of Obstetrics and Gynaecology, GROW-School for Oncology and Developmental Biology, Maastricht, The Netherlands.

Dr. A.J. Kruse, Isala Kliniek, Department Obstetrics and Gynaecology, Zwolle, The Netherlands.

M.G. Lotz, BA, Maastricht University Medical Centre, Department of Nuclear Medicine, Maastricht, The Netherlands.

J.C. van Munsteren, BA, Department of Anatomy and Embryology, Leiden University Medical Center, Leiden, The Netherlands.

I.N.A. Pooters, BA, Maastricht University Medical Centre, Department of Nuclear Medicine, Maastricht, The Netherlands. 
Prof. Dr. M.C. DeRuiter, Department of Anatomy and Embryology, Leiden University Medical Center, Leiden, The Netherlands.

Dr. B.F.M. Slangen, Maastricht University Medical Centre, Department of Obstetrics and Gynaecology, GROW-School for Oncology and Developmental Biology, Maastricht, The Netherlands.

N.N. Smit, MA, Computer Graphics and Visualization, Department of Intelligent Systems, Delft University of Technology, Delft, the Netherlands.

Dr. K.K. Van de Vijver, Antoni van Leeuwenhoek, Dutch Cancer Institute, Amsterdam, The Netherlands.

Drs. T. Wang, General Physician, General Physician practice de Leeuw en Buijs, Weert, The Netherlands 


\section{List of publications}

K De Decker, S Speth, HG ter Brugge, J Bart, LFAG Massuger, M Kleppe, RFPM Kruitwagen, AJ Kruse.

Staging procedures in patients with mucinous borderline tumours of the ovary do not reveal peritoneal or omental disease.

Submitted

S Speth, M Kleppe, RFPM Kruitwagen, INA Pooters, T Van Gorp, BFM Slangen, B Brans. Comparison of intraoperative gamma probe detection and postoperative SPECT/CT imaging of sentinel nodes in ovarian cancer.

J Nucl Med 2016 September, accepted

M Kleppe, MA van der Aa, T Van Gorp, BFM. Slangen, RFPM Kruitwagen.

The impact of lymph node dissection and adjuvant chemotherapy on survival: a nationwide cohort study of patients with clinical early-stage ovarian cancer. Eur J Cancer 2016;66:83-90

M Kleppe, AC Kraima, RFPM Kruitwagen, T Van Gorp, NN Smit, JC van Munsteren, MC DeRuiter.

Understanding lymphatic drainage pathways of the ovaries to predict sites for sentinel nodes in ovarian cancer.

Int J Gynecol Cancer 2015;25:1405-1414.

M Kleppe, B Brans, T Van Gorp, BFM Slangen, AJ Kruse, K.K. van de Vijver, R.F.P.M. Kruitwagen.

Sentinel node bij het ovariumcarcinoom: een fase 1 studie. De SONAR studie.

Nederlands Tijdschrift Obstetrie en Gynaecologie. November 2014.

M Kleppe, B Brans, T Van Gorp, BFM Slangen, AJ Kruse, INA Pooters, MG Lotz, KK Van de Vijver, RFPM Kruitwagen.

The detection of sentinel nodes in ovarian cancer: a feasibility study. 
J Nucl Med 2014;55:1799-1804.

M Kleppe, J Bruls, T Van Gorp, L Massuger, BFM Slangen, K van de Vijver, AJ Kruse, RFPM Kruitwagen.

Mucinous borderline tumours of the ovary and the appendix: a retrospective study and overview of the literature.

Gynecol Oncol 2014;133:155-158.

M Kleppe, LCM Amkreutz, T Van Gorp, BFM Slangen, AJ Kruse, RFPM Kruitwagen.

Lymphnode metastasis in stage I and II sex cord stromal and malignant germ cell tumours of the ovary: a systematic review.

Gynecol Oncol 2014;133:124-127.

M Kleppe, MH van Hooff, JP Rhemrev.

The effect of the total motile sperm count in intra-uterine insemination on the ongoing pregnancy rate.

Andrologia 2014;46:1183-1188.

M Kleppe, T Van Gorp, BFM Slangen, AJ Kruse, B Brans, INA Pooters, KK Van de Vijver, RFPM Kruitwagen.

Sentinel node in ovarian cancer: study protocol for a phase 1 study.

Trials 2013;15;14:47.

M Kleppe, T Wang, T Van Gorp, BFM Slangen, AJ Kruse, RFPM Kruitwagen.

Lymph Node Metastasis in stage I and II ovarian cancer: a review.

Gynecol Oncol 2011;123:610-614.

M Kleppe, CAG Holleboom.

Impetigo Herpetiformis: zwangerschapsdermatose met potentieel ernstige morbiditeit voor moeder en foetus.

Nederlands Tijdschrift Obstetrie en Gynaecologie. May 2010. 


\section{Oral presentations}

M Kleppe, MA van der Aa, T Van Gorp, BFM. Slangen, RFPM Kruitwagen.

The impact of lymph node resection and adjuvant chemotherapy on survival in early-stage ovarian cancer. Data of 3658 patients.

ESGO, International meeting of the European society of gynaecological oncology, Nice, France, October 2015.

M Kleppe, MA van der Aa, T Van Gorp, BFM. Slangen, RFPM Kruitwagen.

De behandeling van vroeg stadium ovariumcarcinoom in Nederland.

Regionale refereeravond, MUMC+, September 2014.

M Kleppe, B Brans, T Van Gorp, BFM Slangen, AJ Kruse, INA Pooters, MG Lotz, KK Van de Vijver, RFPM Kruitwagen.

Sentinel node procedure bij het ovariumcarcinoom: de SONAR studie.

Gynaecongres, Leeuwarden, Mei 2014.

M Kleppe, B Brans, T Van Gorp, BFM Slangen, AJ Kruse, INA Pooters, MG Lotz, KK Van de Vijver, RFPM Kruitwagen.

Sentinel node procedure bij het ovariumcarcinoom: de SONAR studie.

Regionale refereeravond, MUMC+, September 2013.

M Kleppe, J Bruls, T Van Gorp, L Massuger, BFM Slangen, K van de Vijver, AJ

Kruse, RFPM Kruitwagen.

Mucineus borderline tumor en de appendix.

Regionale refereeravond, MUMC+, September 2013.

M Kleppe, T Wang, T Van Gorp, BFM Slangen, AJ Kruse, RFPM Kruitwagen.

Vroeg stadium ovariumcarcinoom en lymfkliermetastasen.

Regionale refereeravond, Orbis Medisch Centrum, Mei 2012.

M Kleppe, T Van Gorp, BFM Slangen, AJ Kruse, RFPM Kruitwagen.

The state of the art treatment of ovarian cancer in the Netherlands. 
Symposium Aken, Genk, Maastricht, MUMC+, Januari, 2012.

M Kleppe, CAG Holleboom.

Zwangerschapsdermatosen: infectieus en niet infectieus. Impetigo herpetoformis en Varicella Zoster.

Regionale refereeravond, Medisch Centrum Haaglanden, November 2009.

\section{Poster presentations}

M Kleppe, AC Kraima, RFPM Kruitwagen, T Van Gorp, NN Smit, JC van Munsteren, MC

DeRuiter.

Understanding lymphatic drainage pathways of the ovaries to predict sites for sentinel nodes in ovarian cancer.

ESGO, International meeting of the European society of gynaecological oncology, Nice, France, October 2015.

M Kleppe, B Brans, T Van Gorp, BFM Slangen, AJ Kruse, INA Pooters, MG Lotz, KK Van de Vijver, RFPM Kruitwagen.

The detection of sentinel nodes in ovarian cancer: a feasibility study.

ESGO, International meeting of the European society of gynaecological oncology,

Oktober 2013, Liverpool, United Kingdom.

M Kleppe, J Bruls, T Van Gorp, L Massuger, BFM Slangen, K van de Vijver, AJ

Kruse, RFPM Kruitwagen.

Mucinous borderline tumours of the ovary and the appendix.

ESGO, International meeting of the European society of gynaecological oncology,

Oktober 2013, Liverpool, United Kingdom.

M Kleppe, T Van Gorp, BFM Slangen, AJ Kruse, RFPM Kruitwagen.

Lymfkliermetastasen in stadium I en II ovarium carcinoom.

Gynaecongres, Papendal, November 2011.

M Kleppe, MH van Hooff, JP Rhemrev. 
Het effect van de total motile count in IUI op het cumulatieve zwangerschapspercentage.

Gynaecongres, Papendal, November 2011.

M Kleppe, T Van Gorp, BFM Slangen, AJ Kruse, RFPM Kruitwagen.

Lymph Node Metastasis in stage I and II ovarian cancer: a review.

ESGO, International meeting of the European society of gynaecological oncology, September 2011, Milan, Italy.

\section{Awards and prizes}

The Alavi-Mandell Award for the publication "The detection of sentinel nodes in ovarian cancer: a feasibility study".

April 2015, American Society of Nuclear Medicine and molecular imaging.

$2^{\mathrm{e}}$ prijs flitspresentaties voor de presentatie "Sentinel node procedure bij het ovarium carcinoom: de SONAR studie".

Mei 2014, Gynaecongres, Leeuwarden. 


\section{Curriculum Vitae}

Marjolein Kleppe werd geboren op 18 november 1981 in Dordrecht. Zij behaalde haar VWO diploma in 2000 aan het Titus Brandsma College, te Dordrecht. In hetzelfde jaar startte ze met de opleiding geneeskunde aan de Universiteit Leiden. Haar wetenschapsstage volgde ze bij de parasitologie waarvoor zij veldonderzoek deed in Ghana. Haar laatste co-schap was een keuze co-schap Emergency Medicine in het Royal Prince Alfred Hospital te Sydney, Australië. Voordat ze ging werken reisde ze door Australië, Nieuw-Zeeland, Fiji en de Cook Islands. Hierna ging ze in 2008 aan de slag als ANIOS in het Bronovo ziekenhuis in Den Haag en vervolgens in het MUMC+. Tijdens haar werk als ANIOS in het MUMC+ startte ze met onderzoek bij gynaecologische oncologie, waarvan het resultaat nu voor u ligt.

In 2012 startte ze met de opleiding tot gynaecoloog in het Zuyderland Medisch Centrum, locatie Sittard, opleiders dr. G.L. Bremer en dr. H.J. Mertens. Het tweede en derde opleidingsjaar volgde ze in het MUMC+, opleiders prof. dr. R.F.P.M. Kruitwagen en dr. G.A.J. Dunselman. Het vierde jaar hervatte zij in het Zuyderland Medisch Centrum, locatie Sittard. Het vijfde jaar van de opleiding werkt ze in het Martini ziekenhuis te Groningen, opleider dr. A. J. Van Loon. Per 1 januari 2017 zal ze het laatste jaar van haar opleiding volgen in het UMCG, opleider prof. dr. S. Scherjon. Ze woont samen met Sabine Boertjens in Groningen. 


\section{Dankwoord}

Het is af! Het is al met al best een werk om die 's' kwijt te raken. Promoveren doe je dan ook niet alleen. Allereest wil ik de patiënten bedanken die deel hebben genomen aan de SONAR studie. Zonder hen zou de studie naar de sentinel node niet mogelijk zijn geweest. Dank daarvoor.

Omdat het dankwoord het beste gelezen stuk is van een proefschrift (of heeft $\mathrm{u}$ wel eerst de voorgaande hoofdstukken gelezen?) wilde ik natuurlijk een mooi dankwoord op schrijven. Ik had me voorgenomen om samen met een glas rode wijn een goede slechte huilfilm te kijken en daarna het dankwoord te gaan schrijven. Deze voorbereiding bleek niet nodig, want op een dinsdagochtend met een kop thee is dit het resultaat geworden.

Geachte professor Kruitwagen, beste Roy, in 2010 was ik als ANIOS in het MUMC naar je toe gegaan omdat ik graag onderzoek wilde doen. Je had nog wel iets liggen, iets waar een semi-arts, aan was begonnen, het hoefde alleen nog maar even opgeschreven te worden. Het ging om een review over de incidentie van lymfkliermetastasen bij vroeg stadium ovariumcarcinoom. Dat was uiteindelijk iets meer werk, dan we beide dachten. Wel zag jij er toen al een goed eerste hoofdstuk voor een boekje in. Ik dacht toen nog, een heel boekje, dat zien we nog wel. Maar goed, je bent natuurlijk niet voor niks professor, want je hebt gelijk gekregen, het boekje is er! Het artikel werd geaccepteerd als poster voor de ESGO in Milaan en daar kreeg jij ook een mail dat het artikel geaccepteerd was voor publicatie. Op diezelfde ESGO, tijdens een uitgebreide Italiaanse lunch met goede wijn en pasta, vroeg jij je af waarom er wel studies naar de sentinel node waren bij andere vormen van gynaecologische kanker, maar niet bij het ovariumcarcinoom. Waarop jij zei dat je al langer speelde met idee om daar een studie naar op te zetten. Dat leek mij ook heel leuk om uit te zoeken en zo werd tussen de prosecco en tagliatelle het idee van de SONAR studie geboren. Onze besprekingen waren soms tussen de poli patiënten door, nog even voordat de overdracht begon of in de wandelgangen: "kijk nog even naar tabel 2". Je was altijd enthousiast, je reageerde altijd heel snel op de stukken die ik toe stuurde. Jij hebt mij enthousiast gemaakt voor onderzoek. Roy ik vond het altijd heel 
prettig met je samen te werken. Dank voor alles wat je voor me gedaan hebt. Ook wil ik je in je rol als opleider danken dat je mij geholpen hebt met de switch naar Groningen.

Geachte dr. van Gorp, beste Toon, ik wil je bedanken voor de fijne samenwerking. Als ik van jou een artikel terug kreeg leek soms wel of alles rood was, maar doordat je kritisch bent, veel kennis hebt en secuur bent werden de stukken er altijd veel beter door. Daarnaast heb ik van jou geleerd hoe je de titel meer 'sexy' kon maken. Naast dat je een goed wetenschapper bent, bewonder ik je als dokter, je bent empatisch, een goede operateur en een goede opleider.

Geachte dr. Slangen, beste Brigitte, bedankt voor het mee helpen opzetten van de SONAR studie en de kritische blik. In de kliniek viel me op hoe je rustig je blijft bij lastige ingrepen en hoe goed je dat oplost. Ik bewonder hoe je je klinische werk, wetenschap, activiteiten binnen de beroepsvereniging combineert met je gezin. Vooral dat laatste is ook een goed voorbeeld voor de AIOS.

Ik wil de beoordelingscommissie bedanken voor het kritisch beoordelen van mijn manuscript en de Corona voor de oppositie tijdens de promotie.

Beste dr. Brans, beste Boudewijn, jij hebt mij laten kennis maken met de nucleaire geneeskunde. Je straalde als het over straling ging. Bedankt voor de prettige samenwerking.

Geachte dr. Krüse, beste Arnold, dank voor de leuke samenwerking zowel in de wetenschap als in de kliniek. Jouw humor kon ik altijd waarderen. Ik hoop dat we elkaar in het Noorden nog eens tegen komen.

Aan alle medeauteurs dank voor de inzet, dankzij jullie werden de artikelen beter.

Beste Ivo en Maartje, dank voor de voorbereidingen van de nucleaire tracers en het meedenken in het opzetten van de SONAR studie. 
Geachte dr. van der Aa, beste Maaike, veel dank voor de analyses en de figuren. Ik denk dat je af en toe helemaal tureluurs werd van al die nieuwe vragen en andere figuren die we wilden. Maar het resultaat mag er zijn! Heel veel dank voor de fijne samenwerking.

Geachte dr. van de Vijver, beste Koen, bedankt voor de fijne samenwerking. Maar vooral ook voor de gezelligheid tijdens congressen. Jouw enthousiasme voor de pathologie, maakt dat het absoluut geen dode boel is om coupes te bekijken.

Professor de Ruiter en Annelot Kraima, dank voor de fijne samenwerking en gezellige tijd op het lab in Leiden.

Stéphanie heel veel succes met de SONAR 2 studie.

Beste maatschap uit Bronovo ziekenhuis, jullie hebben mij begeleid in de eerste stappen binnen het vakgebied. Ik kijk nog altijd terug op een hele gezellige tijd binnen een leuke club!

Stafleden, verpleging, ondersteund personeel uit MUMC+, veel dank voor de prettige samenwerking. AIOS uit mijn Maastricht tijd, dank voor de lol in de assistentenkamer en daarbuiten op de VrijMiBo!

Beste maatschap van het Zuyderland Medisch Centrum, locatie Sittard-Geleen, dank voor de gezellige tijd en vooral ook leerzame tijd. Ik zal nooit meer over een mooi CTG spreken, tenzij ik het CTG zo mooi vind dat ik hem in een lijst aan de muur wil hangen. Verloskundigen, secretaressen, verpleging, dank voor de fijne samenwerking. Assistenten uit Sittard: Ewka, Fleur, Lieke, Jonas, Guusje, Desiree, Rogier, Natascha, Violet en Lilian, bedankt voor de gezellige tijd!

Beste maatschap, assistenten, verloskundigen, verpleging en mannenborrel van het Martini Ziekenhuis, bedankt voor het opnemen van die AIOS uit het Zuiden, voor het vertrouwen, de vele leermomenten en de fijne sfeer op de werkvloer. 
Ik wil mijn cordial bedanken voor alle leuke momenten samen in de 16 jaar waarin we lief en leed delen.

Eva en Maarten, dank voor de vriendschap. Bij jullie thuis is er altijd plek voor mij met gezelligheid en vooral lekker eten! Met de bakfiets met Pauline en Jabob (en straks ook Simon) naar het strand, was heerlijk om uit te waaien en bij te kletsen.

Eloise, ondanks dat je ver weg op je rots zit en we elkaar niet zo vaak zien, ben ik wel heel blij met jou als vriend. Ik denk nog vaak terug aan de tijd dat ik coschappen liep en na een dag ziekenhuis bij jou thuis op de bank na het eten ongegeneerd in slaap kon vallen. Ook kon ik eindeloos lang ziekenhuisverhalen vertellen zonder dat je het zat werd. Maar natuurlijk ook aan samen duiken op je rots, Bonaire en Thailand. Het wordt weer eens tijd voor een Buddy check.

Eline, Ilse, Annelieke, vanaf jaar 1 samen in de werkgroep bij geneeskunde, door dezelfde beginletter van de achternaam (behalve die ene extra met een W). In de MaPo dagdroomden we over onze toekomst als dokters. Inmiddels heeft iedereen haar weg gevonden door het land heen. Gelukkig na 16 jaar is er altijd nog wel tijd voor een weekend in een huisje ergens in het land met kolonisten, chips, bier, pindakaas met hagelslag, foute muziek en misschien een korte wandeling. Dank voor de vriendschap al die jaren en op naar het volgende weekend!

Britsclub uit Maastricht wat een gezelligheid met jullie. De eerste tijd in Maastricht was voor mij meteen een stuk aangenamer door de VrijMiBo's, Preuvenemint, (ambtenaren) carnaval en oud en nieuw in de Ardennen met jullie. Dank daarvoor!

Ammie! Lieve Lieke, je bent mijn favoriete $2^{\mathrm{e}}$ auteur. Dankzij jou heb ik kunnen kennismaken met de De Kirchröatsjer Damezietsóng. Limburgerser wordt het niet! Ik hoop dat we nog lang gynaecongres kamer matties blijven! 
Tournoike, lieve Fleur, bedankt voor je verhelderende kijk op dingen en je functie als personal shopper heb ik ook zeer gewardeerd!

Lieve Violet, toen we allebei nog niet in opleiding waren en in Den Haag werkten hadden we het plan om een echo apparaat te 'lenen' uit het ziekenhuis en expats te gaan screenen in Afrika. Ik het ochtendspreekuur, jij de middag en dan een beetje duiken en cocktails drinken. Dit plan liep in gedachten altijd spaak bij de oversized bagage balie op Schiphol. Maar het liep uiteindelijk anders want we kwamen beide in Maastricht in opleiding. Jij wilde na je opleiding weer terug naar het Noorden, mij beviel het wel in het bourgondische Zuiden. Nu blijf jij waarschijnlijk wel hangen in het Zuiden en ik ben vertrokken naar het Noorden. Een gevalletje 'life is what happens to you while you are busy making other plans'. Dank voor alle flauwe grappen en goede humor tijdens de bubbelsavondjes!

Lieve Natte Tassen, wat moet een mens zonder een Natte Tas? Bij jullie is er altijd wel een fles bubbels, snorren, een hoogslaper en een luisterend oor. Jullie kijk op het leven, levensvreugde, relativeringsvermogen, bewonder ik enorm. Waren er op de wereld maar meer Natte Tassen! Dan werd er ook een stuk meer gemountainbiked! Lieve Sietze en Natascha (zodat mensen ook weten dat jullie ouders nog een andere naam voor jullie hadden uitgekozen) bedankt voor wie jullie zijn.

From Ass! Lieve Lilian, Japan was een once in a life time experience! Ik heb veel meer van jou geleerd dan alleen gynaecologie. Er is ook zo veel meer dan het werk. "Als ik de liefde kon vangen kreeg iedereen een flesje vol. Dan dronken we de wereld mooi en proosten we op de lol." Bedankt voor je lieve vriendschap, de gezelligheid en leuke gesprekken over het leven buiten werk. En ook voor het mij omhoog duwen op de fiets op de Kruisberg.

Lieve Anne, dank voor de zoete inval op de WP. Naast lange diensten ben je gelukkig altijd in voor leuke dingen zoals het Snorrenfeest, de Amstel Gold race, 
mountainbike tochtjes, cava's drinken op het preuvemint. Het was allemaal hele goede afleiding.

Lieve Jonas, vanaf dag 1 samen in de opleiding en sindsdien zijn we opleidingsmatties. We hebben allebei hetzelfde gevoel voor humor en ook vaak aan één blik genoeg! Jonas ik vind het heel jammer dat we het staartje van de opleiding niet in hetzelfde ziekenhuis afmaken. Standaard aan het eind van de werkdag checkte ik altijd even met jou hoe je dag was en wat jij allemaal beleefd had. Samen met jou de fertiliteitstage was één grote gezelligheid. Ook al ben je vanaf dag 1 mijn opleidingsmattie en gaan we straks de Vulva Clinics starten, was jij de eerste die zei dat ik mijn hart moest volgen naar Groningen. Dank daarvoor. En fijn dat je tijdens de promotie achter me staat als paranimf.

Lieve familie, bedankt voor jullie betrokkenheid en interesse.

Piet en Sylvia, dank voor het besluit om na 3 kinderen toch nog voor een vierde te gaan, anders was Sabine er nooit geweest. En al het goede komt in drieën! Lieve Janneke, Janine en Clara, Selwin en schoonfamilie bedankt voor de gezelligheid in Groningen en Friesland.

Lieve Wouter, jij bent de beste grote broer! Samen met je zusje van 17 jaar naar Afrika, dat was een super gave ervaring! Ondanks dat jij al de medische verhalen maar vies vindt, ben ik wel heel blij en trots dat jij straks achter mij staat. En hoe je natuurlijk daarnaast ook altijd achter me staat.

Lieve pap en mam, bedankt voor jullie onvoorwaardelijke steun en liefde.

Bedankt voor de geweldige jeugd en mede door jullie ben ik nu hier.

Lieve Sabine, met jou is het elke dag een klein feestje! Ik bewonder hoe je in het leven staat en tegen de dingen aankijkt, het glas is half vol-mentaliteit en je relativeringsvermogen. Ik ben ontzettend blij met jou samen te zijn, ik hou van je! 
